\title{
Warm gas towards young stellar objects in Corona Australis
}

\section{Herschel/PACS observations from the DIGIT key programme ${ }^{\star}$}

\author{
Johan E. Lindberg ${ }^{1,2}$, Jes K. Jørgensen ${ }^{2,1}$, Joel D. Green ${ }^{3}$, Gregory J. Herczeg ${ }^{4,5}$, Odysseas Dionatos ${ }^{1,2,6}$, \\ Neal J. Evans II ${ }^{3}$, Agata Karska ${ }^{5}$, and Susanne F. Wampfler ${ }^{1,2}$ \\ ${ }^{1}$ Centre for Star and Planet Formation, Natural History Museum of Denmark, University of Copenhagen, \\ Øster Voldgade 5-7, 1350 København K, Denmark \\ e-mail: jlindberg@snm.ku.dk \\ 2 Niels Bohr Institute, University of Copenhagen, Juliane Maries Vej 30, 2100 København Ø, Denmark \\ 3 The University of Texas at Austin, Department of Astronomy, 2515 Speedway, Stop C1400, Austin TX 78712-1205, USA \\ ${ }^{4}$ Kavli Institute for Astronomy and Astrophysics, Peking University, 100871 Beijing, PR China \\ 5 Max Planck Institute for Extraterrestrial Physics, Postfach 1312, 85741 Garching, Germany \\ ${ }^{6}$ University of Vienna, Department of Astronomy, Türkenschanzstrasse 17, 1180 Vienna, Austria \\ Received 1 July 2013 / Accepted 27 November 2013
}

\section{ABSTRACT}

\begin{abstract}
Context. The effects of external irradiation on the chemistry and physics in the protostellar envelope around low-mass young stellar objects are poorly understood. The Corona Australis star-forming region contains the R CrA dark cloud, comprising several low-mass protostellar cores irradiated by an intermediate-mass young star.

Aims. We study the effects of the irradiation coming from the young luminous Herbig Be star R CrA on the warm gas and dust in a group of low-mass young stellar objects.

Methods. Herschel/PACS far-infrared datacubes of two low-mass star-forming regions in the R CrA dark cloud are presented. The distributions of $\mathrm{CO}, \mathrm{OH}, \mathrm{H}_{2} \mathrm{O}$, [C II], [O I], and continuum emission are investigated. We have developed a deconvolution algorithm which we use to deconvolve the maps, separating the point-source emission from the extended emission. We also construct rotational diagrams of the molecular species.

Results. By deconvolution of the Herschel data, we find large-scale (several thousand AU) dust continuum and spectral line emission not associated with the point sources. Similar rotational temperatures are found for the warm CO (282 $\pm 4 \mathrm{~K})$, hot CO (890 $\pm 84 \mathrm{~K})$, $\mathrm{OH}(79 \pm 4 \mathrm{~K})$, and $\mathrm{H}_{2} \mathrm{O}(197 \pm 7 \mathrm{~K})$ emission in the point sources and the extended emission. The rotational temperatures are also similar to those found in other more isolated cores. The extended dust continuum emission is found in two ridges similar in extent and temperature to molecular millimetre emission, indicative of external heating from the Herbig Be star R CrA.

Conclusions. Our results show that nearby luminous stars do not increase the molecular excitation temperatures of the warm gas around young stellar objects (YSOs). However, the emission from photodissociation products of $\mathrm{H}_{2} \mathrm{O}$, such as $\mathrm{OH}$ and $\mathrm{O}$, is enhanced in the warm gas associated with these protostars and their surroundings compared to similar objects not subjected to external irradiation.
\end{abstract}

Key words. stars: formation - ISM: individual objects: R CrA - ISM: molecules - astrochemistry

\section{Introduction}

One of the open questions in low-mass star formation is how the irradiation from intermediate-mass stars affects the chemistry, temperature, and excitation conditions in the warm gas around low-mass young stellar objects. With the resolution of the Herschel Space Observatory, superior to that of previous farinfrared (FIR) telescopes, in combination with deconvolution algorithms, we can now address this question. Both the spectral line emission from the gas and the dust continuum emission from the warm regions peak in the FIR part of the electromagnetic spectrum. The second most abundant molecule in the interstellar medium (ISM) after $\mathrm{H}_{2}$ is $\mathrm{CO}$, and it has a large number of transitions in this band. In addition, water and its related species, $\mathrm{OH}$, have their most important transitions in this band.

With the advent of the Herschel Space Observatory (Pilbratt et al. 2010), FIR observations with unprecedented spatial and spectral resolution have been made available. The Herschel

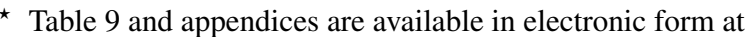
http://www. aanda.org
}

observations of low-mass young stellar objects (YSOs) reveal numerous lines of $\mathrm{CO}, \mathrm{H}_{2} \mathrm{O}$, and $\mathrm{OH}$, along with atomic lines like [O I] and [C II] (e.g. Herczeg et al. 2012; Kristensen et al. 2012; Green et al. 2013). In most of the sources studied, the CO rotational diagrams can be fitted with warm and hot components, with rotational temperatures of about $300 \mathrm{~K}$ and $900 \mathrm{~K}$, respectively (Green et al. 2013; Karska et al. 2013; Manoj et al. 2013). The $\mathrm{OH}$ and $\mathrm{H}_{2} \mathrm{O}$ emission is usually characterised by somewhat lower rotational temperatures around $100 \mathrm{~K}$ (Goicoechea et al. 2012; Herczeg et al. 2012; Wampfler et al. 2013). Most of these studies of low-mass YSOs have targeted isolated embedded objects. To better understand low-mass star formation in more dynamic environments, studies of small groups of resolvable embedded objects are warranted.

This paper presents PACS (Photodetector Array Camera and Spectrometer; Poglitsch et al. 2010) maps of the R Coronae Australis (R CrA) dark cloud, which harbours one of the closest star-forming regions, located at a distance of $130 \mathrm{pc}$ (Neuhäuser $\&$ Forbrich 2008). The cloud is named for the young star R CrA, which has spectral classifications ranging from F5 to B5 (e.g. 
F5: Hillenbrand et al. 1992; A0: Manoj et al. 2006; B8: Bibo et al. 1992; B5: Gray et al. 2006). The cloud was mapped in CO by Loren (1979), who found an elongated cloud covering about 2 pc by $0.5 \mathrm{pc}$ and peaking near R CrA. Higher resolution maps of $\mathrm{C}^{18} \mathrm{O}$ with the Swedish-ESO Submillimetre Telescope (SEST) revealed several dense molecular clumps with masses between $2 M_{\odot}$ and $50 M_{\odot}$ near R CrA (Harju et al. 1993). Surveys of the clumps have revealed a number of embedded protostars, with IRS7 in the clump to the southeast of R CrA and IRS5 in the clump to the west. Taylor \& Storey (1984) report that IRS7 is the most reddened source in the region, with a visual extinction of more than 25 mag. Brown (1987) split IRS7 into two sources separated by $14^{\prime \prime}$, using VLA $6 \mathrm{~cm}$ observations. For a more complete description and references, see Neuhäuser \& Forbrich (2008).

The $\mathrm{R}$ CrA region (including IRS7) has been studied at FIR wavelengths using the ISO telescope (Lorenzetti et al. 1999; Giannini et al. 1999), detecting lines from CO, OH, O I, and C II. However, these studies were limited by the ISO angular resolution of $80^{\prime \prime}$, which made it impossible to separate the sources in the region. Sicilia-Aguilar et al. (2013) present $100 \mu \mathrm{m}$ and $160 \mu \mathrm{m}$ photometry maps of the CrA region observed with Herschel/PACS. The IRS7 clump is positioned with its centre between the two Herbig Ae/Be stars R CrA and $\mathrm{T} \mathrm{CrA}$, and harbours a handful of Class 0/I Young Stellar Objects (YSOs). Nutter et al. (2005) report detections of four cores within the IRS7 and IRS5 clumps in SCUBA $450 \mu \mathrm{m}$ and $850 \mu \mathrm{m}$ data: SMM 1A, with no mid-infrared (MIR) counterpart, proposed to be a pre-stellar core; SMM 1B, coincident with the MIR source IRS7B; SMM 1C, a Class 0 protostar; and SMM 4, coincident with the IRS5 clump. Peterson et al. (2011) report SMA $1.3 \mathrm{~mm}$ point-source continuum detections at the positions of SMM 1B (IRS7B), SMM 1C, and SMM 4 (IRS5N). The IRS5 clump contains two protostellar sources, IRS5A and IRS5N, and is situated at a slightly greater projected distance from R CrA than is IRS7. The source IRS5A is not detected in SMA $1.3 \mathrm{~mm}$ continuum observations, whereas IRS5N shows significantly fainter continuum emission than the IRS7 sources (95 mJy in IRS5N, $320 \mathrm{mJy}$ in IRS7B; Peterson et al. 2011).

Through single-dish APEX observations, Schöier et al. (2006) found elevated $\mathrm{H}_{2} \mathrm{CO}$ and $\mathrm{CH}_{3} \mathrm{OH}$ abundances and rotational temperatures in IRS7A and IRS7B, which were suggested to be caused by increased internal heating or outflows. Van Kempen et al. (2009a) used mid- $J$ CO observations to find an EW outflow centred at IRS7A, but also found that the CO line fluxes in the region are too high to originate from heating by the embedded protostars, and proposed that the heating originates from R CrA. This was confirmed by Lindberg \& Jørgensen (2012), who found large-scale ( $10000 \mathrm{AU}) \mathrm{H}_{2} \mathrm{CO}$ emission heated to $40-60 \mathrm{~K}$ by external irradiation from R CrA. The IRS5 sources are found to be less affected by the irradiation from $\mathrm{R} \mathrm{CrA} \mathrm{(the} \mathrm{H}_{2} \mathrm{CO}$ rotational temperature in a $29^{\prime \prime}$ beam is $47 \mathrm{~K}$ for IRS7B and $28 \mathrm{~K}$ for IRS5A; Lindberg et al., in prep.).

In Sect. 2 we will describe the methods of observations and data reductions and show the first results. A discussion of our deconvolution algorithm and the results thereof follows in Sect. 3. In Sect. 4 we investigate models of the excitation conditions of the gas. In Sect. 5 we discuss the interpretations of our results, and in Sect. 6 we list our conclusions.

\section{Observations and data description}

In this section we first describe the observations and methods of data reduction. A more detailed description can be found in
Green et al. (2013). We then discuss the first results of these reductions.

\subsection{Observation setup and data reduction}

Integral-field spectroscopy observations in the FIR regime that cover parts of the R CrA star-forming region were performed by the Photodetector Array Camera and Spectrometer (PACS; Poglitsch et al. 2010) on board the ESA Herschel Space Observatory (Pilbratt et al. 2010), with a spectral range from $55 \mu \mathrm{m}$ to $210 \mu \mathrm{m}$. The observations were carried out in range-spectroscopy mode. The spectral resolution varies between $0.013 \mu \mathrm{m}$ and $0.13 \mu \mathrm{m}\left(\Delta v \approx 55-318 \mathrm{~km} \mathrm{~s}^{-1}, \lambda / \Delta \lambda \approx\right.$ 950-5500).

These observations are part of the Dust, Ice, and Gas in Time (DIGIT; PI: N. Evans) open-time key programme, a survey of the change of FIR spectral features through the evolution of young stellar objects. For details of the DIGIT key programme, see Green et al. (2013). The observed PACS fields are shown compared to the point-source positions and a Spitzer $4.5 \mu \mathrm{m}$ image in Fig. 1, and the telescope pointings are found in Table 1. The configuration of the 9.' $4 \times 99^{\prime} 4$ spaxels shown in Fig. 1 is a simplified model: in reality, no spaxels overlap. This is taken into account in the deconvolution method described in Sect. 3.1.

The spectral line fluxes in each spaxel were calculated using the HIPE 8.0.2489 reduction of the data, but corrected by the continuum value in the HIPE 6.1 reduction. The HIPE 8.0.2489 reduction was found to provide on average a $40 \%$ better signalto-noise ratio (spanning from almost no improvement up to about a factor of 2 at specific wavelengths), whereas the HIPE 6.1 reduction produced more accurate and reliable spectral energy distributions (SEDs) by $\sim 20 \%$. These SEDs are consistent with PACS photometry within $\sim 10 \%$ (Green et al. 2013). The method of combining these two reductions is thoroughly described in Green et al. (2013). For the continuum flux densities, the HIPE 6.1 reduction was used.

For each spectral line in each spaxel, a first-degree polynomial baseline calculated from the surrounding line-free channels was subtracted from the spectrum. The total line flux was then calculated by summing the channel flux densities within a typical linewidth (mostly $0.1-0.5 \mu \mathrm{m}$ ) and multiplying this sum by the channel width. Of the $\mathrm{OH}$ doublets, only four out of nine are resolved. In the unresolved cases, the combined total flux for both $\mathrm{OH}$ lines is calculated, and half of this value is used for each of the components in the rotational diagram analysis. The statistical errors are calculated from the rms noise around each spectral line (any lines weaker than $3 \sigma$ are ignored) to get the total standard deviation of the spectral line flux. If nothing else is stated, all errors given in this paper are at the $1 \sigma$ level. In addition to the statistical errors, a systematic calibration error at $20 \%$ of the flux (see Green et al. 2013) is used when calculating quantities such as the rotational temperatures and total number of molecules from the rotational diagrams.

The two bands below $103 \mu \mathrm{m}$ are considerably noisier than the bands above this wavelength, and between approximately $94 \mu \mathrm{m}$ and $103 \mu \mathrm{m}$ the noise makes line fluxes very difficult to estimate. The spectrometer is affected by leakage in the wavelength ranges $70-73 \mu \mathrm{m}, 98-105 \mu \mathrm{m}$, and above $190 \mu \mathrm{m}$, which produces ghost images of lines from the next higher grating order. Fluxes at these wavelengths are thus less reliable than at other wavelengths (Herczeg et al. 2012).

In addition to the Herschel data, we also used the ISIS spectrograph on the William Herschel Telescope (WHT) on 6 August 2012 to obtain a low-resolution $(R \sim 1000)$ optical spectrum of 


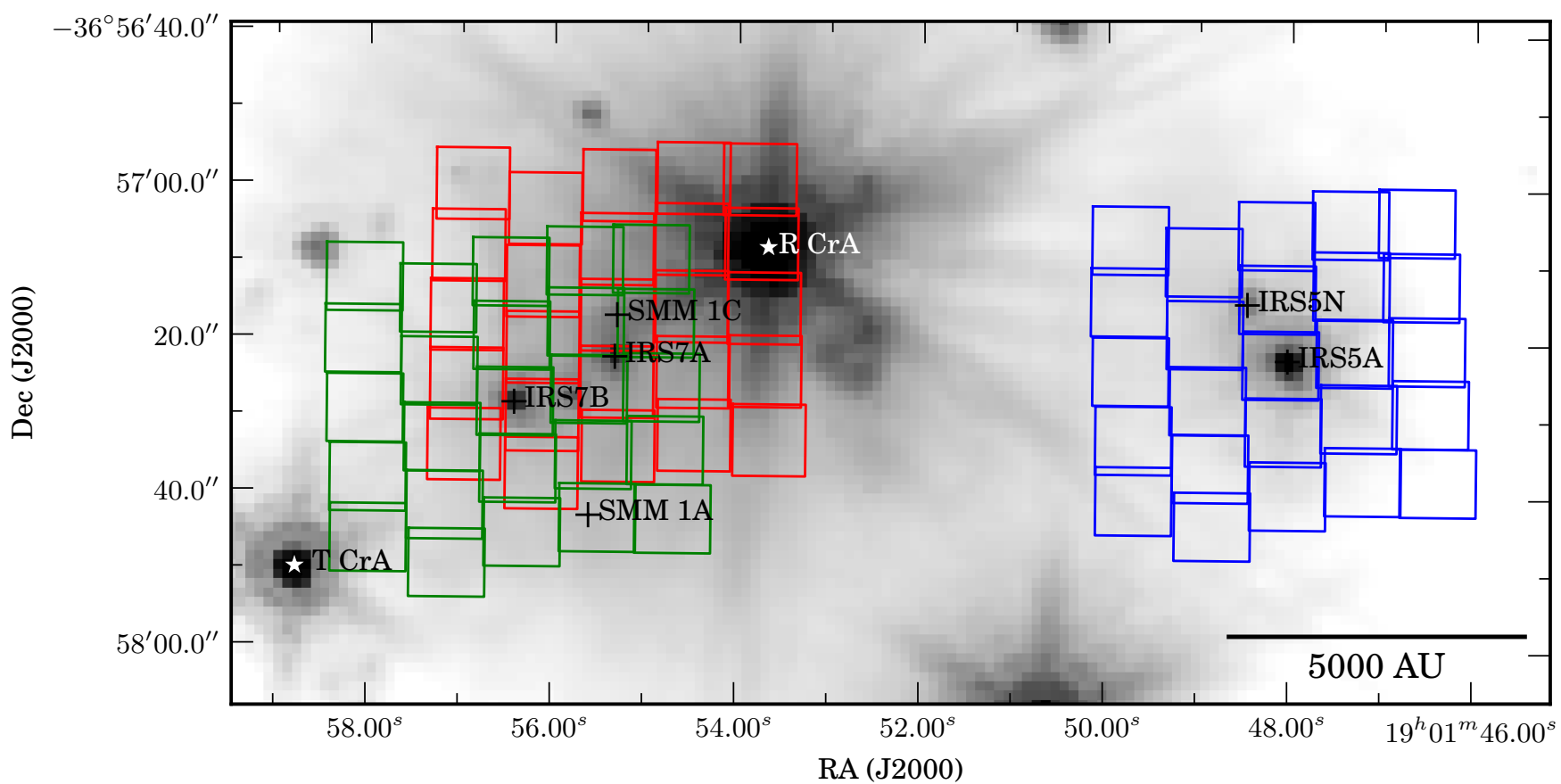

Fig. 1. Schematic overview of the three PACS footprints, centred at SMM 1C (red), IRS7B (green), and IRS5A (blue), overlaid on a Spitzer $4.5 \mu \mathrm{m}$ image. The spaxel positions as recorded by PACS on the sky are at the centre of each spaxel, and each spaxel measures 9.' $4 \times 9$ '. 4 in this representation.

Table 1. Observational parameters.

\begin{tabular}{lllrll}
\hline \hline Region & $\begin{array}{l}\text { RA } \\
(\mathrm{J} 2000.0)\end{array}$ & $\begin{array}{l}\text { Dec } \\
(\mathrm{J} 2000.0)\end{array}$ & \multicolumn{1}{l}{$\begin{array}{l}\mathrm{PA}^{a} \\
\left.{ }^{\circ}\right]\end{array}$} & Observing IDs & Date of observation \\
\hline IRS7A & $19: 01: 55.3$ & $-36: 57: 17.0$ & -0.22 & 1342206990,1342206989 & $2010-10-23$ \\
IRS7B & $19: 01: 56.4$ & $-36: 57: 28.3$ & 2.47 & 1342207807,1342207808 & $2010-11-02$ \\
IRS5A & $19: 01: 48.1$ & $-36: 57: 22.7$ & 2.40 & 1342207806,1342207805 & $2010-11-01$ \\
\hline
\end{tabular}

Notes. Positions give the centre coordinates of the central spaxel. All footprints are $5 \times 5$ spaxel grids with approximately 9 '. 25 separation between the spaxel centres. ${ }^{(a)}$ Position angle with respect to an equatorial grid coordinate system.

R CrA covering 3000-10000 A. The spectrum was flux calibrated against the spectrophotometric standard LTT 7987. The analysis of this spectrum will be discussed in Sect. 4.1.1.

\subsection{First look}

In this section, we provide a first look at the PACS continuum and spectral maps before they are treated by our deconvolution algorithm.

\subsubsection{Continuum maps}

The total emission continuum maps of IRS7 and IRS5 at three representative wavelengths can be found in Figs. 2 and 3, and continuum maps at $20 \mu \mathrm{m}$ steps can be found in Figs. A.1 and A.2. These continuum maps show extended emission of the order of 30-60" in size. The larger sizes are found in the longer wavelengths (which could be suggested to be attributed only to the larger beam size at these wavelengths, but the shapes of the emission suggest differently; see also Sect. 3.2). The highest continuum flux densities in the strongest illuminated spaxels are found around $85 \mu \mathrm{m}$ in IRS7 and $105 \mu \mathrm{m}$ in IRS5, but the spatially integrated flux density is strongest around $120 \mu \mathrm{m}$ in IRS7 and $155 \mu \mathrm{m}$ in IRS5 (since the longer wavelength data have more extended PSFs, which spreads the signal over a larger solid angle). It is, however, difficult to come to any conclusions about the stages of evolution of the individual sources from the total emission, since it is a combination of several compact sources and any extended emission. For a study of this kind, deconvolution of the emission is necessary.

The continuum emission in the IRS7 region is more extended than the line emission (see Sect. 2.2.2), and is detected across most of the two PACS fields that cover the region (see Figs. 2 and 3). Without any deconvolution, it is impossible to distinguish the point sources detected in MIR and mm data (IRS7A, IRS7B, SMM 1C, and R CrA).

The IRS5 core consists of two separate sources, of which the northern source IRS5N is detected in SMA continuum, whereas the southern IRS5A is detected in Spitzer continuum and line emission. It is difficult to distinguish the two sources in the PACS data.

\subsubsection{Spectral line maps}

The sum of the PACS spectra of the spaxels closest to IRS7A and SMM 1C is shown in Fig. 4. The detected species are indicated 

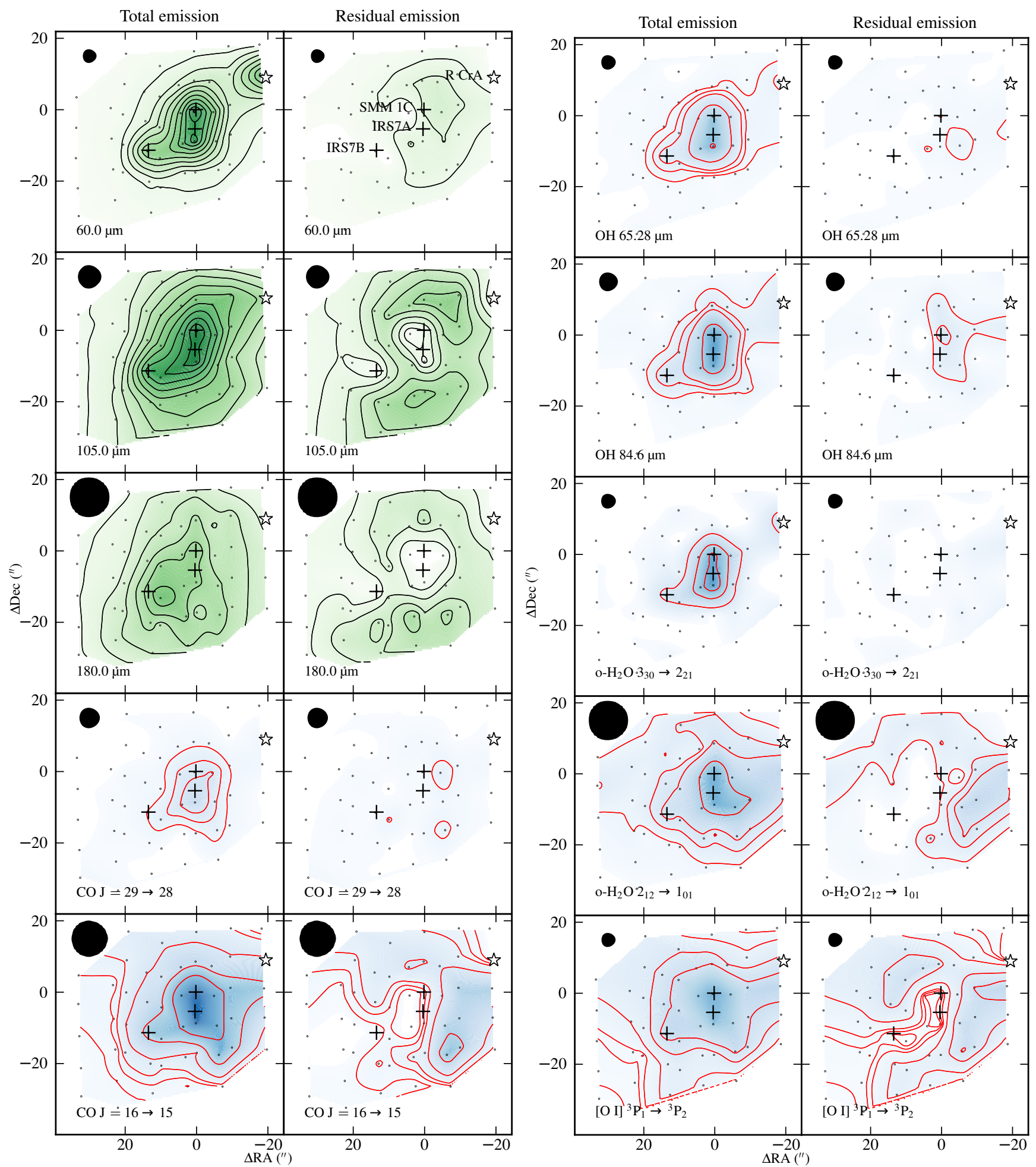

Fig. 2. IRS7 contour maps of continuum emission at three wavelengths (green with black contours), and some important spectral lines (the same as in Table 4 of Green et al. 2013, blue with red contours). The first column in each panel shows the total emission, whereas the second column in each panel shows the residual emission after deconvolution (emission not associated with the point sources). These maps of the whole band of continuum and all spectral lines can be found in Appendices A and B. The point sources used for the deconvolution are marked with crosses, except for R CrA which is marked with a star symbol (the sources are identified in the top row). The dots indicate the PACS spaxel centres. The Herschel PSF for each observation is shown in the top left corner. Contour levels are at $10 \mathrm{Jy}$ for the continuum maps, and at $5 \sigma, 10 \sigma, 15 \sigma, 30 \sigma$, $60 \sigma$, and $90 \sigma$ for the line maps. The blue colour maps have the same ranges for each separate molecule, with maximum fluxes of $7 \times 10^{-16} \mathrm{~W} \mathrm{~m}^{-2}$ for $\mathrm{CO}, 1 \times 10^{-15} \mathrm{~W} \mathrm{~m}^{-2}$ for $\mathrm{OH}, 4 \times 10^{-16} \mathrm{~W} \mathrm{~m}^{-2}$ for $\mathrm{o}-\mathrm{H}_{2} \mathrm{O}$, and $1 \times 10^{-14} \mathrm{~W} \mathrm{~m}^{-2}$ for $[\mathrm{OI}]$.

by different colours. The total emission spectral line emission maps of some important lines are found in Figs. 2 and 3, and all spectral line emission maps can be found in Figs. B.1-B.10. The
CO emission in IRS7 is generally found to be more extended than the $\mathrm{OH}$ and $\mathrm{H}_{2} \mathrm{O}$ emission. The emission from the atomic species C II and O I detected in IRS7 is even more extended. In 
J. E. Lindberg et al.: Warm gas towards young stellar objects in Corona Australis
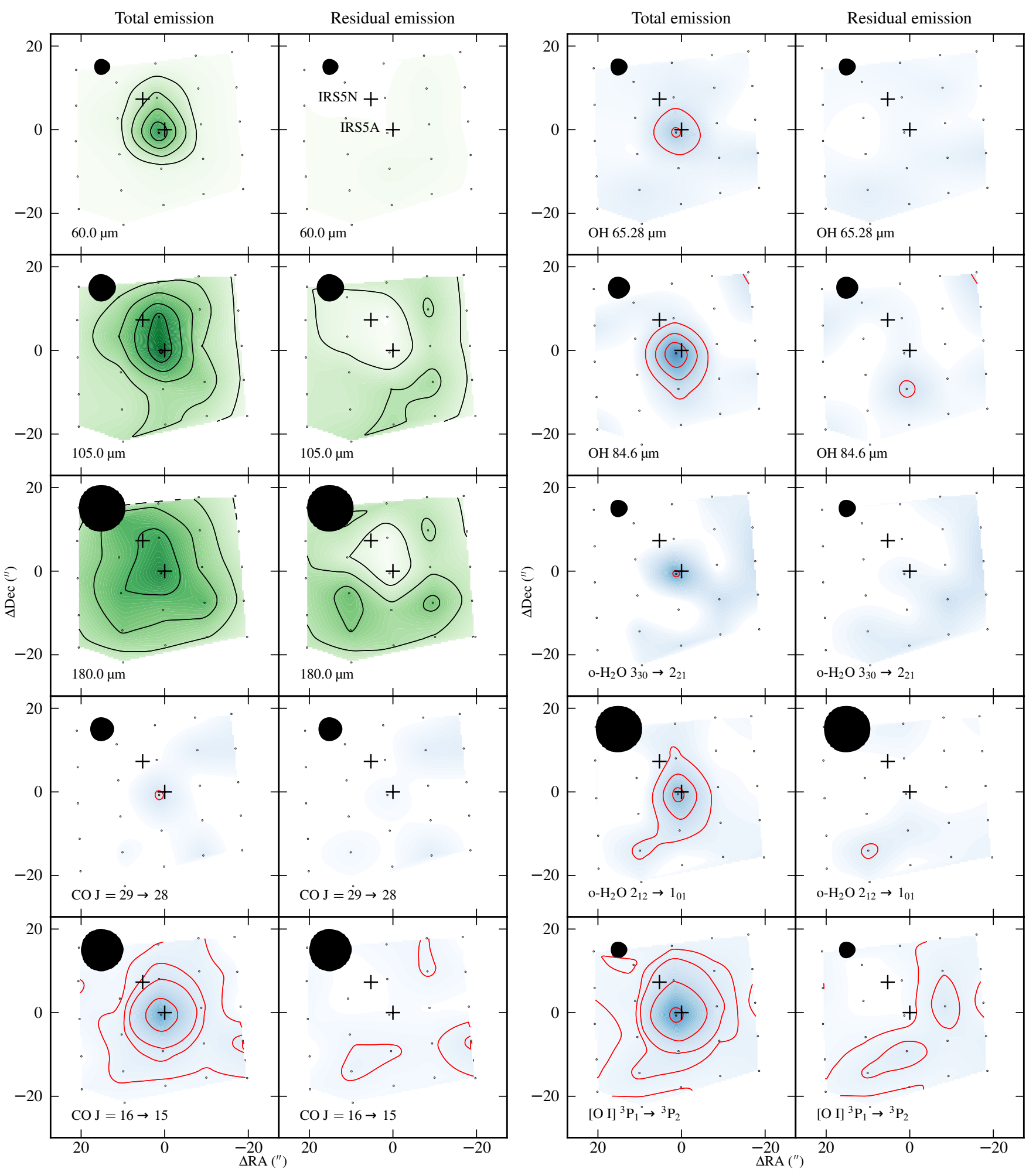

Fig. 3. As in Fig. 2, but for IRS5. Contour levels are at 3 Jy for the continuum maps, and as in Fig. 2 for the line maps. The blue colour maps have the same ranges for each separate molecule, with maximum fluxes of $1.5 \times 10^{-16} \mathrm{~W} \mathrm{~m}^{-2}$ for $\mathrm{CO}, 2 \times 10^{-16} \mathrm{~W} \mathrm{~m} \mathrm{~m}^{-2}$ for $\mathrm{OH}, 1 \times 10^{-16} \mathrm{~W} \mathrm{~m}{ }^{-2}$ for $\mathrm{o}-\mathrm{H}_{2} \mathrm{O}$, and $2 \times 10^{-15} \mathrm{~W} \mathrm{~m}^{-2}$ for $[\mathrm{OI}]$.

particular, the [C II] line emission peaks off-source in a position east of IRS7A. Compared to the emission from $\mathrm{CO}, \mathrm{OH}$, and $\mathrm{H}_{2} \mathrm{O}$ in the IRS7 cloud, which is mainly centred on the three point sources, the [C II] emission in IRS7 is more extended in the EW-direction. The [OI] emission is similar to the $\mathrm{CO}$ and $\mathrm{OH}$ emission, but apparently much stronger in SMM 1C than in
IRS7A. There is also a relatively strong band of extended emission in a band $\mathrm{W}$ and NE of IRS7A. For IRS5, the spectral line emission appears unresolved.

The $\mathrm{CO}, \mathrm{OH}$, and $\mathrm{H}_{2} \mathrm{O}$ emission detected by PACS in the IRS7 field is centrally peaked on the three protostellar sources in the IRS7 field (SMM 1C, IRS7A, and IRS7B), with the strongest 


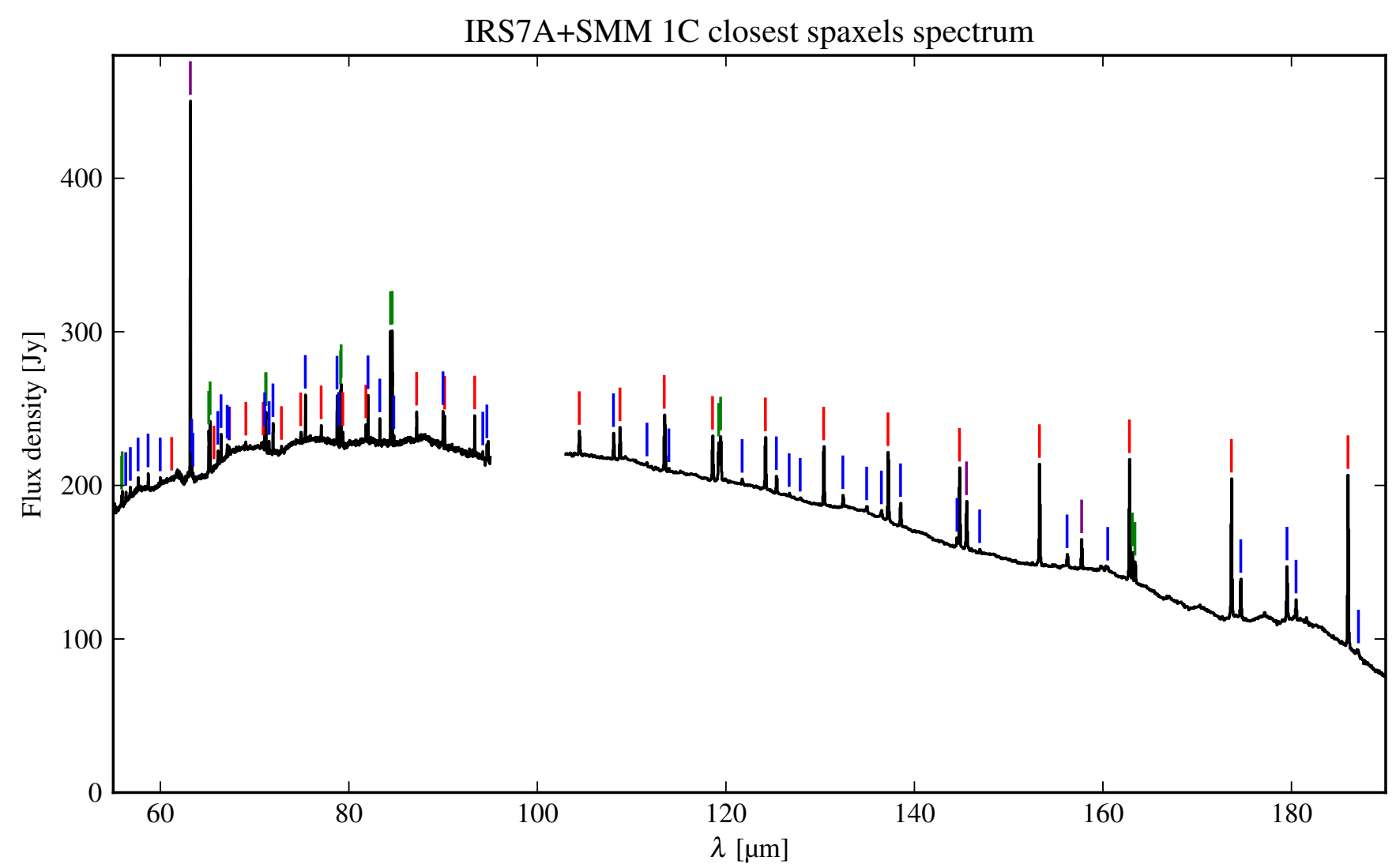

Fig. 4. Sum of the spectra of the spaxels closest to IRS7A and SMM 1C. No deconvolution or correction factors have been applied to the spectrum. It thus appears fainter in the red part compared to the true spectrum. The coloured vertical lines indicate the detected spectral line species: $\mathrm{CO}$ (red), $\mathrm{OH}$ (green), $\mathrm{H}_{2} \mathrm{O}$ (blue), and atomic species (purple).

emission from SMM 1C and IRS7A. This is consistent with the centrally peaked (but also extended) $\mathrm{CO} J=6 \rightarrow 5$ and $\mathrm{HCO}^{+}$ emission found by van Kempen et al. (2009a,b), but different from the very extended $\mathrm{H}_{2} \mathrm{CO}$ and $\mathrm{CH}_{3} \mathrm{OH}$ emission detected in SMA (Submillimeter Array) and APEX (Atacama Pathfinder Experiment) observations (Lindberg \& Jørgensen 2012). Very little or no FIR line emission is seen around SMM 1A, although this region shows very strong $\mathrm{H}_{2} \mathrm{CO}$ and $\mathrm{CH}_{3} \mathrm{OH}$ emission in the SMA mm data.

\section{Image analysis}

As is clearly seen in the total emission continuum and line maps (Figs. 2 and 3), the emission originating from the different sources in the IRS7 and IRS5 fields cannot be easily separated, and a deconvolution method needs to be applied. To test the hypothesis that most of the emission can be accounted for by the previously known point sources, we need to deconvolve the data with the point spread function (PSF) of the observations.

\subsection{Deconvolution method}

The effective diameter of the Herschel Space Observatory is $3.28 \mathrm{~m}$. The Herschel PSF is slightly triangular projected on the sky because of the three-point mount of the telescope dish (see Fig. C.1), but it can be roughly approximated by a Gaussian with a full width at half maximum (FWHM) between $4^{\prime \prime}$ and $12^{\prime \prime}$ depending on the wavelength. For our deconvolution algorithm we instead use PSFs constructed from the simulated telescope PSF (see Appendix C). The PACS spectrometer detector array consists of 25 spaxels, positioned in a square $5 \times 5$ pattern. The separations between the spaxel centres are on average approximately 9.'25. This has the effect that emission from a point source will spill over to adjacent spaxels, particularly for the longer wavelengths, since the telescope PSF will be larger than the spaxel size. This is normally easy to correct for when observing a single well-centred point source, because it is then in principle sufficient to use the central spaxel flux (or flux density) multiplied by a wavelength-dependent PSF correction factor $^{1}$. However, if observing off-centred point sources, several point sources in the same field, or extended emission (or combinations of these), the interpretation of the data becomes more difficult. We find several point sources in the IRS7 field, some of them not well-centred on spaxel centres, as well as signs of extended emission in the PACS field, and we therefore need another method to separate the emission from the different possible origins. The IRS7 field was observed in two partially overlapping PACS pointings, which creates an almost Nyquist-sampled map for all but the shortest wavelengths in the overlap region.

To be able to distinguish point-source emission from more extended emission, the signal must be deconvolved from the PSF. However, the spatial resolution of the signal is limited by the design of the PACS instrument, only providing 25 data points for this signal per pointing. We have developed a method that can still provide deconvolution of point sources from the PACS

\footnotetext{
1 See the PACS manual v. 2.4 for a detailed description of the PSF correction factor and the PACS beam: http://herschel. esac. esa. int/Docs/PACS/html/pacs_om.html
} 
Table 2. Continuum point sources in MIR and/or (sub)mm data.

\begin{tabular}{llll}
\hline \hline Name & $\begin{array}{l}\text { RA } \\
(\mathrm{J} 2000.0)\end{array}$ & $\begin{array}{l}\text { Dec } \\
(\mathrm{J} 2000.0)\end{array}$ & Other names \\
\hline IRS7A $^{a}$ & $19: 01: 55.33$ & $-36: 57: 22.4$ & IRS7W, IRS7 \\
SMM 1C $^{a}$ & $19: 01: 55.31$ & $-36: 57: 17.0$ & SMA 2, Brown 9 \\
IRS7B $^{a}$ & $19: 01: 56.42$ & $-36: 57: 28.4$ & $\begin{array}{l}\text { IRS7E, SMM 1B, } \\
\end{array}$ \\
& & & SMA 1 \\
CXO 34 $^{a}$ & $19: 01: 55.78$ & $-36: 57: 27.9$ & FP-34 \\
R CrA $^{b}$ & $19: 01: 53.67$ & $-36: 57: 08.0$ & \\
IRS5A $^{b}$ & $19: 01: 48.03$ & $-36: 57: 22.2$ & CrA-19, IRS5ab \\
IRS5N $^{c}$ & $19: 01: 48.47$ & $-36: 57: 14.9$ & CrA-20, SMM 4, \\
& & & FP-25 \\
\hline
\end{tabular}

Notes. ${ }^{(a)}$ Coordinates from ALMA $0.8 \mathrm{~mm}$ continuum map (Lindberg et al. 2013). ${ }^{(b)}$ Coordinates from Spitzer $4.5 \mu \mathrm{m}$ image data (Peterson et al. 2011). ${ }^{(c)}$ Coordinates from SMA $1.3 \mathrm{~mm}$ maps (Peterson et al. 2011).

data, called POMAC ${ }^{2}$. The method is based on the CLEAN algorithm (Högbom 1974), often used to deconvolve undersampled maps in radio interferometry. Because of the low number of data points, our method relies on a priori knowledge about the point-source positions. In this case, we employ ALMA data from Lindberg et al. (2013), and SMA and Spitzer data from Peterson et al. (2011) to establish the positions of the YSOs with an accuracy of $\lesssim 1^{\prime \prime}$. Based on the larger sample of more isolated sources (Green et al. 2013), we expect that a major part of the emission seen with Herschel, both continuum and line, will originate from the point sources seen at mid-infrared and submillimetre wavelengths, and we use the deconvolution to test this hypothesis. The CLEAN algorithm is used, but has been modified so that it is only allowed to identify these pre-defined point sources as sources of emission. The algorithm then iterates over the PACS data with customary break criteria (such as avoiding subtraction below the noise floor in any spaxel). After this, the residual map can be studied to identify previously unknown point sources as well as extended emission. Repeating the process after adding new point sources will eventually leave all extended emission in the residual map (however, still convolved with the PSF). The algorithm was tested on the PACS spectrometer continuum of the disc source HD 100546 (Sturm et al. 2010), which is not expected to differ significantly from a point source in the continuum. The deconvolution produced results within errors of those obtained using the PACS PSF correction factor across the whole PACS band, and no significant residuals were noted. A more detailed explanation of the POMAC algorithm, including a description of how the telescope PSFs were generated, can be found in Appendix C.

\subsubsection{Definition of point sources}

The sources treated in this paper have been studied in several previous papers, and have been given many different names. To facilitate comparison with other work, a list of the most common names for these point sources found in the literature can be found in Table 2. We will maintain the name usage of Lindberg \& Jørgensen (2012).

To match the line and continuum emission with compact objects we rely on a priori position data from other observations with better resolution obtained in other wavebands. To

\footnotetext{
2 Poor Man's CLEAN.
}

cover all sources that could be visible in the FIR, we used both longer (submillimetre) and shorter (mid-infrared) wavelength observations to identify possible point sources. Peterson et al. (2011) used SMA $226 \mathrm{GHz}$ continuum observations to identify four continuum peaks in the IRS7 and IRS5 regions (IRS7B, SMA2/SMM 1C, IRS5N, and R CrA); and Spitzer $4.5 \mu \mathrm{m}$ observations to find five continuum peaks in the same fields ( $\mathrm{R} \mathrm{CrA}$, CrA-19/IRS5A, IRS7B, IRS7A, and CXO 34). We identified four of these sources (IRS7B, SMM 1C, IRS7A, and CXO 34) in ALMA Cycle 0 observations of the $342 \mathrm{GHz}$ continuum centred at IRS7B (Lindberg et al. 2013). The ALMA coordinates are of superior accuracy, and will be used for these sources. The coordinates for these sources can be found in Table 2 .

From these point-source positions, we selected the six most prominent sources (only excluding CXO 34, which is almost an order of magnitude weaker at $4.5 \mu \mathrm{m}$ than the second weakest infrared source) to use for the deconvolution of both the continuum and the line emission. The excluded source CXO 34 is not only very weak, but also situated between the much stronger sources IRS7B and IRS7A, and it would not be possible to disentangle any FIR emission originating from CXO 34 from the emission of the surrounding sources.

The sources IRS7A and SMM 1C have an angular separation of only $5^{\prime \prime}$, i.e. not spatially resolved by the PACS array. We attempted to separate the emission from IRS7A and SMM 1C, but we found that the results were unreliable, even for the shorter wavelengths. Therefore, we have decided to treat IRS7A and SMM 1C together, summing the fluxes given for the two sources. This is not ideal, however, since it has been suggested that they are of different types (Class 0 and Class I, respectively). Since both the line emission and the continuum emission in this region are extended in the north-south direction it is very likely that both sources contribute to this emission.

\subsection{Results of the continuum deconvolution}

Deconvolved and non-deconvolved spectra of the point sources are found in Fig. 5. When comparing the non-deconvolved and deconvolved spectra, we find that the spectral lines are stronger than the continuum in the deconvolved data (especially in the IRS7 sources), indicating that the spectral line emission is less extended than the continuum emission, a pattern which is also seen in the larger DIGIT sample of embedded objects (hereby referred to as the DIGIT sample; Green et al. 2013), although data of more crowded regions (e.g. Serpens; Dionatos et al. 2013) or strong outflow sources (e.g. L1448-MM; Lee et al. 2013) show spatial extent. As in the other sources in the DIGIT sample, the emission from $[\mathrm{OI}]$ is more extended than that from the other species. We also find that IRS5N only shows a few spectral lines, but has a continuum as strong as that of IRS5A. The bumps in the spectra around $180 \mu \mathrm{m}$ are caused by an instrument leakage effect.

The continuum deconvolution is performed on averaged linefree spectral boxes every $5 \mu \mathrm{m}$. These results are compared to the continuum level found in the channel-by-channel deconvolution (see above and Fig. 5) to check the consistency, and the two methods are found to be in good agreement.

The total emission maps and the deconvolution residual maps at three different wavelengths can be found in the first six panels in Figs. 2 and 3. Continuum total emission maps and residual maps in $20 \mu \mathrm{m}$ steps can be found in Figs. A.1 and A.2.

Running the POMAC deconvolution algorithm on the continuum data leaves strong residual emission in a shape that remains constant across most of the PACS spectral band, except for 

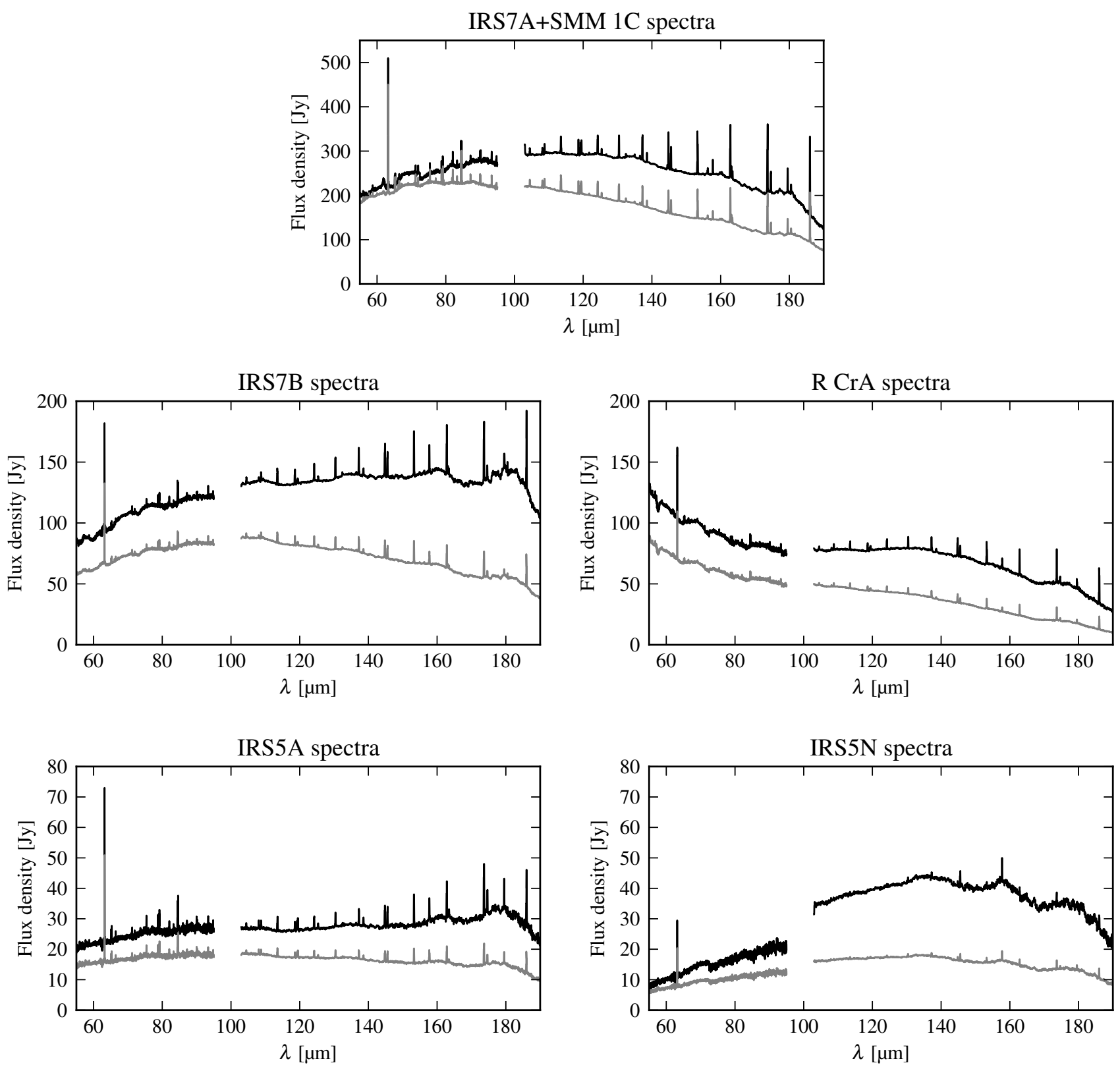

Fig. 5. Spectra of the deconvolved point-source emission (black) and the non-deconvolved data (grey). We note that since the flux density in the line channels are sums of the line and continuum flux densities, these will not be properly cleaned (line strengths are under-estimated in these spectra owing to the more extended continuum). The deconvolved spectra shown here are used to check for consistency with the continuum deconvolution (see Sect. 3.2), but not for extracting line fluxes (see Sect. 3.3 for a description of the method used for the line flux deconvolution).

the shortest wavelengths. We only find a little continuum residual emission in the centre of the IRS7 field, but strong residual emission is found in two ridges north and south of the YSOs. This could be an effect of the stop criteria used by the POMAC algorithm; these criteria assume that all emission on the pointsource positions originates from the point sources, and should thus not leave considerable residuals on-source. We did several POMAC runs with different stop criteria. None of them could reproduce a smooth continuum residual, but they either showed the ridges or left significant emission on the point source positions. The continuum residual at $110 \mu \mathrm{m}$ is shown in Fig. 6, which is used to define the residual regions Res N-c and Res S-c (c for continuum). These regions are chosen to coincide with the spaxels with a residual continuum spectral flux density of at least $30 \mathrm{Jy}$ at $110 \mu \mathrm{m}$. Also shown are the ridges of molecular gas detected in $\mathrm{H}_{2} \mathrm{CO}$ and $\mathrm{CH}_{3} \mathrm{OH}$ (Lindberg \& Jørgensen 2012), which bear resemblance in shape and position to the PACS continuum residuals. The Res S-c region coincides partially with the pre-stellar core SMM 1A detected in SCUBA submm observations (Nutter et al. 2005). These similarities indicate that the deconvolution algorithm and the stop criteria used produce accurate results. Some residual continuum emission is found also in the IRS5 field, but only at wavelengths longer than $100 \mu \mathrm{m}$.

\subsection{Results of the spectral line deconvolution}

For the deconvolution of the spectral lines, the flux of each spectral line in each spaxel is first measured after the subtraction of linear baselines, producing total line flux maps. These total line flux maps are then the input data to the POMAC algorithm. The 


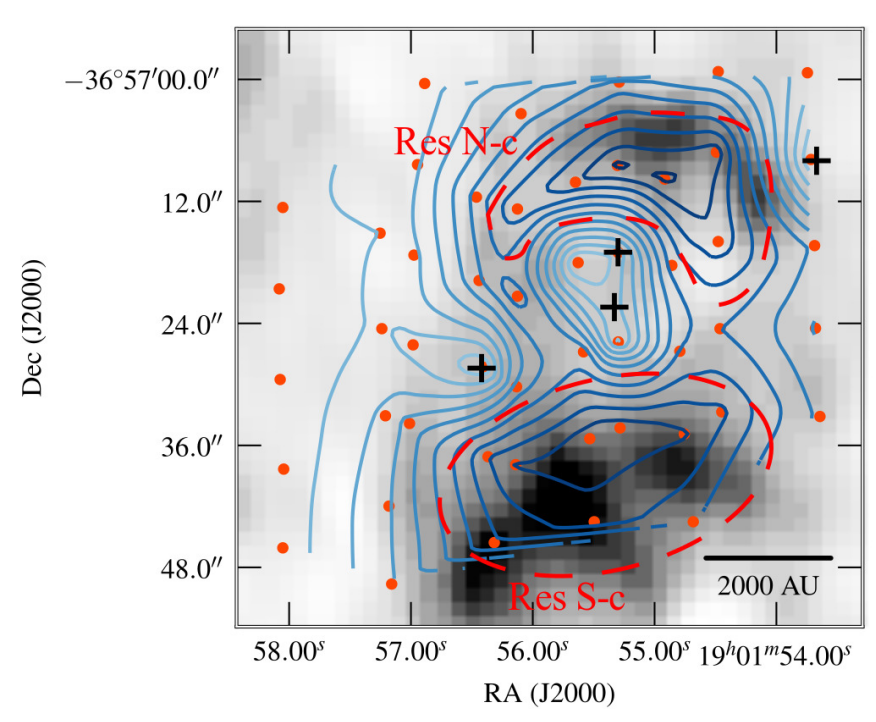

Fig. 6. The $110 \mu \mathrm{m}$ continuum residual map (coloured contours) of IRS7 overplotted on the $\mathrm{H}_{2} \mathrm{CO}_{303} \rightarrow 2_{02}$ emission (greyscale) from SMA+APEX observations (Lindberg \& Jørgensen 2012). The orange dots show the PACS spaxel centres. Contour levels are at $5 \mathrm{Jy}$ intervals and go from light blue to dark blue as the level of emission increases (i.e. the residual emission is at a minimum near the centre of the figure). The red dashed lines illustrate our definition of the two extended (residual) ridge regions (Res N-c and Res S-c) in IRS7 continuum. The crosses show the point sources used for the deconvolution.

line strengths are somewhat higher (approximately 20\%) than those found when extracting line strengths from the continuumsubtracted channel-by-channel-deconvolved spectra (Fig. 5). This is due to the stop criteria, making the algorithm reach the noise floor earlier in the channel-by-channel data than in the line flux data, since the signal-to-noise $(\mathrm{S} / \mathrm{N})$ level is lower for the individual channels than for the total line fluxes.

The line flux maps and the deconvolution residual maps of seven important spectral lines can be found in the last 14 panels in Figs. 2 and 3. All total line intensity maps and residual maps can be found in Figs. B.1 and B.10 in Appendix B. The extracted point source line fluxes are listed in Table 9. We note that, as in the case of the continuum deconvolution, the POMAC algorithm will attribute all on-source emission to the point sources, and not leave residuals at the point-source positions. One could also assume that the point sources are sitting on a plateau of extended emission, but attempts to model that situation with different stop criteria have been unsuccessful. Nevertheless, since the residual emission is much fainter than the point-source emission, this solution would not change the results of excitation analysis of the point sources dramatically. It is difficult to give an exact estimate of this contribution, since the residual emission is primarily found west of the IRS7 point sources, and a smooth distribution of the extended emission around these sources is thus not possible. For $\mathrm{CO}$, the molecule with the most prominent extended emission, the point source line fluxes would be $\lesssim 25 \%$ lower assuming a flat distribution of the extended emission as strong as the residual west of the point sources, but it should be noted that this is a worst-case scenario. Our estimates show that the errors in the rotational temperatures (Sect. 4.2) would increase by $\sim 60 \%$ assuming this scenario. The reported extended emission should, on the other hand, be seen as a lower limit on the amount of extended gas.
Some spectral lines are heavily affected by line blending, and will not be considered in the additional analysis. These are listed in Table 3. In addition, only spectral lines between $55 \mu \mathrm{m}$ and $100 \mu \mathrm{m}$, and $103 \mu \mathrm{m}$ and $195 \mu \mathrm{m}$ are considered, because of leakage and/or a high noise level in the outer parts of the bands. The instrument is also affected by leakage in the ranges 70 $73 \mu \mathrm{m}$ and $98-105 \mu \mathrm{m}$, so line strengths in these bands are less reliable than those in other bands.

\subsubsection{CO emission patterns}

We find CO emission associated with all the pre-defined point sources in the deconvolution, although only very faint emission is found to originate from IRS5N.

Studying the residual maps (Figs. 6 and B.1-B.2), considerable extended $\mathrm{CO}$ emission is found southwest, east, north, and west of the point sources in the IRS7 field for the lower$J$ COlines $(J \lesssim 25)$. The $\mathrm{S} / \mathrm{N}$ is too low to detect more than traces of higher- $J$ CO lines, but this will be discussed further in Sect. 4.2.2. In the IRS5 field, the CO emission is completely point-like, leaving no residuals after the deconvolution.

The relatively strong extended emission in the SW part could also be explained by a $\mathrm{CO}$ point source in a position not associated with any YSO. No corresponding point source has been identified in the SMA data, in the Spitzer data, or in any source catalogue, but we cannot rule out that this is a very faintly emitting YSO. However, we do not consider this to be a point source, and the excitation conditions of this emission will be treated together with that of the other extended CO emission in Sect. 4.2.2. This peak bears resemblance to an outflow front, but it does not align well with the EW outflow found by van Kempen et al. (2009a). The origin is thus uncertain.

The CO line emission in IRS5 is well-centred on the spaxel corresponding to IRS5A (see CO emission maps and CO residual maps in Figs. 3 and B.2), and when running the POMAC code on this data assuming IRS5A as the only point source, only marginal residuals are found. It is thus reasonable to believe that IRS5N produces only a very low amount of $\mathrm{CO}$ emission.

\subsection{2. $\mathrm{OH}$ and $\mathrm{H}_{2} \mathrm{O}$ emission patterns}

The line emission from $\mathrm{OH}$ and $\mathrm{H}_{2} \mathrm{O}\left(\mathrm{p}-\mathrm{H}_{2} \mathrm{O}\right.$ and o- $\left.\mathrm{H}_{2} \mathrm{O}\right)$ will be treated in the same section because of their similar emission patterns. The sources IRS7A and SMM 1C are strong emitters of lines from these molecules, whereas IRS7B is much weaker in the $\mathrm{OH}$ and $\mathrm{H}_{2} \mathrm{O}$ transitions. The $\mathrm{OH}, \mathrm{p}-\mathrm{H}_{2} \mathrm{O}$, and $\mathrm{o}-\mathrm{H}_{2} \mathrm{O}$ line emission maps and residual maps are found in Figs. B.3-B.8. There is less extended emission in the $\mathrm{OH}$ and $\mathrm{H}_{2} \mathrm{O}$ line data than in the $\mathrm{CO}$ data; in particular, there is no considerable extended emission southwest of IRS7. There is, however, some extended $\mathrm{OH}$ and $\mathrm{H}_{2} \mathrm{O}$ emission west of IRS7.

As in the case of CO, IRS5A seems to be the dominant emitter of $\mathrm{OH}$ and $\mathrm{H}_{2} \mathrm{O}$ in the IRS5 field; IRS5N does not contribute with any significant emission.

\subsubsection{Atomic line emission patterns}

Compared to the $\mathrm{CO}, \mathrm{OH}$, and $\mathrm{H}_{2} \mathrm{O}$ emission in the IRS7 cloud, which is mainly centred on the three point sources, the [C II] emission is more extended in the EW direction and peaks in different positions from the other spectral lines in the IRS7 field. However, the [C II] PACS data of protostellar sources are often affected by emission in the off-positions. We have 
Table 3. Spectral lines in the observed spectral band which are not considered in the analysis since they are affected by line blending.

\begin{tabular}{lllll}
\hline \hline Species & Transition & $\begin{array}{l}\text { Wavelength } \\
{[\mu \mathrm{m}]}\end{array}$ & Blend & Transition \\
\hline $\mathrm{o}-\mathrm{H}_{2} \mathrm{O}$ & $6_{25} \rightarrow 5_{14}$ & 65.2 & $\mathrm{OH}$ & ${ }^{2} \Pi_{3 / 2}(J=9 / 2-\rightarrow 7 / 2+)$ \\
$\mathrm{CO}$ & $J=35 \rightarrow 34$ & 74.9 & $\mathrm{o}-\mathrm{H}_{2} \mathrm{O}$ & $7_{25} \rightarrow 6_{34}$ \\
$\mathrm{o}-\mathrm{H}_{2} \mathrm{O}$ & $7_{25} \rightarrow 6_{34}$ & 74.9 & $\mathrm{CO}$ & $J=35 \rightarrow 34$ \\
$\mathrm{CO}$ & $J=31 \rightarrow 30$ & 84.4 & $\mathrm{OH}$ & ${ }^{2} \Pi_{3 / 2}(J=7 / 2+\rightarrow 5 / 2-)$ \\
$\mathrm{OH}$ & ${ }^{2} \Pi_{3 / 2}(J=7 / 2+\rightarrow 5 / 2-)$ & 84.4 & $\mathrm{CO}$ & $J=31 \rightarrow 30$ \\
$\mathrm{o}-\mathrm{H}_{2} \mathrm{O}$ & $6_{25} \rightarrow 6_{16}$ & 94.6 & $\mathrm{o}-\mathrm{H}_{2} \mathrm{O}$ & $4_{41} \rightarrow 432$ \\
$\mathrm{o}-\mathrm{H}_{2} \mathrm{O}$ & $4_{41} \rightarrow 4_{32}$ & 94.7 & $\mathrm{o}-\mathrm{H}_{2} \mathrm{O}$ & $6_{25} \rightarrow 6_{16}$ \\
$\mathrm{CO}$ & $J=23 \rightarrow 22$ & 113.4 & $\mathrm{o}-\mathrm{H}_{2} \mathrm{O}$ & $4_{14} \rightarrow 3_{03}$ \\
$\mathrm{o}-\mathrm{H}_{2} \mathrm{O}$ & $4_{14} \rightarrow 3_{03}$ & 113.5 & $\mathrm{CO}$ & $J=23 \rightarrow 22$ \\
$\mathrm{p}-\mathrm{H}_{2} \mathrm{O}$ & $3_{22} \rightarrow 3_{13}$ & 156.2 & $\mathrm{o}-\mathrm{H}_{2} \mathrm{O}$ & $5_{23} \rightarrow 4_{32}$ \\
$\mathrm{o}-\mathrm{H}_{2} \mathrm{O}$ & $5_{23} \rightarrow 4_{32}$ & 156.3 & $\mathrm{p}-\mathrm{H}_{2} \mathrm{O}$ & $3_{22} \rightarrow 3_{13}$ \\
\hline
\end{tabular}

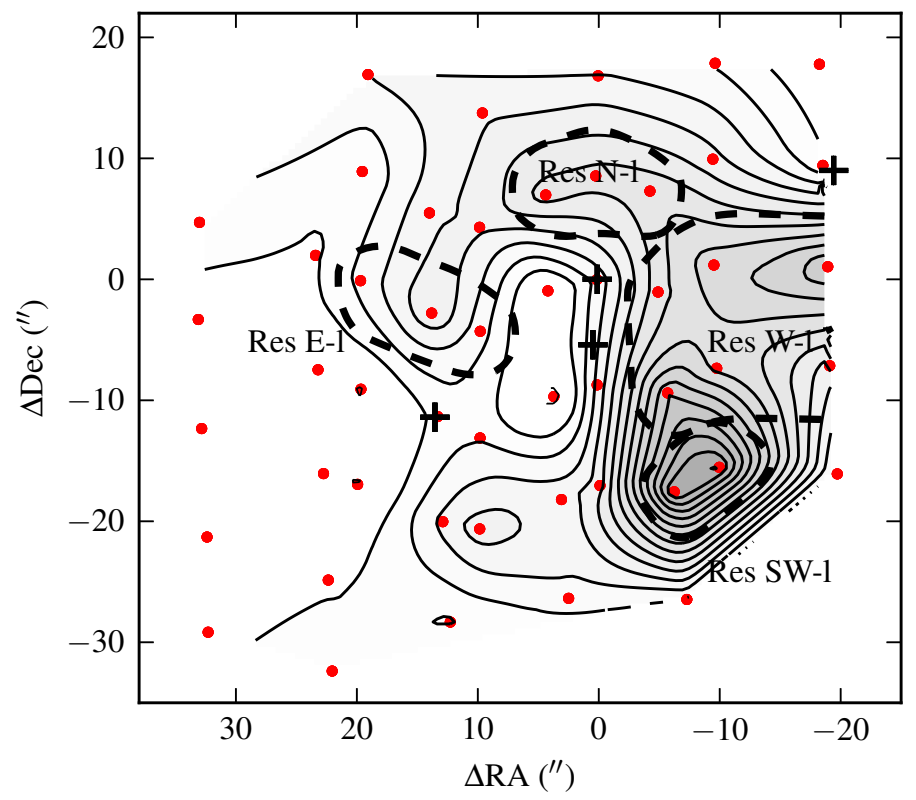

Fig. 7. The dashed lines illustrate our definition of the four extended (residual) regions Res SW-1, Res E-1, Res N-1, and Res W-1 in IRS7 $\mathrm{CO}$ and $\mathrm{OH}$. The contour map shows the $\mathrm{CO} J=19 \rightarrow 18$ residual map. The red dots show the PACS spaxel centres. Contours are $3 \sigma=$ $1.55 \times 10^{-17} \mathrm{~W} \mathrm{~m}^{-2}$.

investigated the signal using the two different nod positions, and the general structure is similar but not identical in these two data sets, which suggests that the detected [C II] morphology may partly be an observational effect. The [C II] data could thus be unreliable, and will not be discussed further.

The [O I] emission peaks on the point sources, but also shows strong extended emission in the whole IRS7 field, with residual peaks similar to those of $\mathrm{OH}$ and $\mathrm{H}_{2} \mathrm{O}$, indicating that they trace the same extended gas. This is a good indicator of largescale photo-dissociation region (PDR) activity or, alternatively, outflow-associated shocks, since $\mathrm{H}_{2} \mathrm{O}$ can be photo-dissociated into $\mathrm{OH}$ and $\mathrm{O}$ (Hollenbach \& Tielens 1997). The [O I] data are not affected by off-position emission to a significant level. The line ratio between the $145 \mu \mathrm{m}$ and $63 \mu \mathrm{m}$ [O I] lines varies between 0.06 and 0.11 for the point sources, which is also indicative of a strong radiation field (i.e. PDR activity; Kaufman et al. 1999).

Around IRS5, however, the [C II] and [O I] emission can be explained by two point sources centred at IRS5A and IRS5N.

\subsection{Comparing line and continuum emission}

Using our deconvolution algorithm POMAC, we find that the FIR line emission mostly originates from the (sub)mm/MIR continuum point sources (but there is also some $\mathrm{CO}$ and $\mathrm{OH}$ line emission from residual regions: Res SW-1, Res E-1, Res N-1, and Res W-1), whereas the FIR continuum shows a much more extended shape (see Fig. 6). After using the deconvolution algorithm we find that most of the continuum emission not associated with point sources can be found in two ridges extending in the east-west direction, positioned north and south of the YSO point sources. These ridges coincide with molecular $\left(\mathrm{H}_{2} \mathrm{CO}\right.$ and $\mathrm{CH}_{3} \mathrm{OH}$ ) emission detected in millimetre data. It has been proposed that these ridges are heated by external irradiation from the Herbig Be star R CrA (Lindberg \& Jørgensen 2012).

The northern ridge (Res N-c and Res N-l) coincides not only with the northern $\mathrm{H}_{2} \mathrm{CO}$ ridge observed by Lindberg \& Jørgensen (2012), but also to some extent with $\mathrm{HCO}^{+} J=3 \rightarrow 2$ emission (Groppi et al. 2007) and X-ray emission (Forbrich \& Preibisch 2007). Thus, another possible explanation of the physical conditions found in the region could be that the gas is dominated by X-ray irradiation.

\section{Analysis}

\subsection{Analysis of the spectral energy distributions}

The SEDs of the low-mass point sources, can be found in Fig. 8, where continuum data points from Spitzer and SCUBA measurements as well as the deconvolved Herschel/PACS spectra have been included. Bolometric luminosities are calculated from integration of a first degree spline fit to the SED data points (including line-free points across the Herschel spectrum) and bolometric temperatures are calculated from the mean frequencies of these spline fits (see Myers et al. 1998). The results are shown in Table 4. For the sources with strong submm emission, the SCUBA data have been linearly extrapolated to allow for integration of the SEDs up to $1.3 \mathrm{~mm}$ (cf. Jørgensen et al. 2009). The SED of R CrA is treated in Sect. 4.1.1.

The luminosities of the observed low-mass sources (IRS7A+SMM 1C, IRS7B, IRS5A, and IRS5N) are all of the order of $1-10 L_{\odot}$. All these sources fulfil the $L_{\text {bol }} / L_{\text {submm }}<200$ criterion for Class 0 sources (André et al. 1993). Nonetheless, the low-mass sources all have bolometric temperatures that fall in the Class I range, except IRS5N, which is a Class 0 source. However, as discussed previously, IRS7A+SMM 1C is a binary unresolved by Herschel, where the components have very different MIR and submm SEDs. It is thus likely that the 

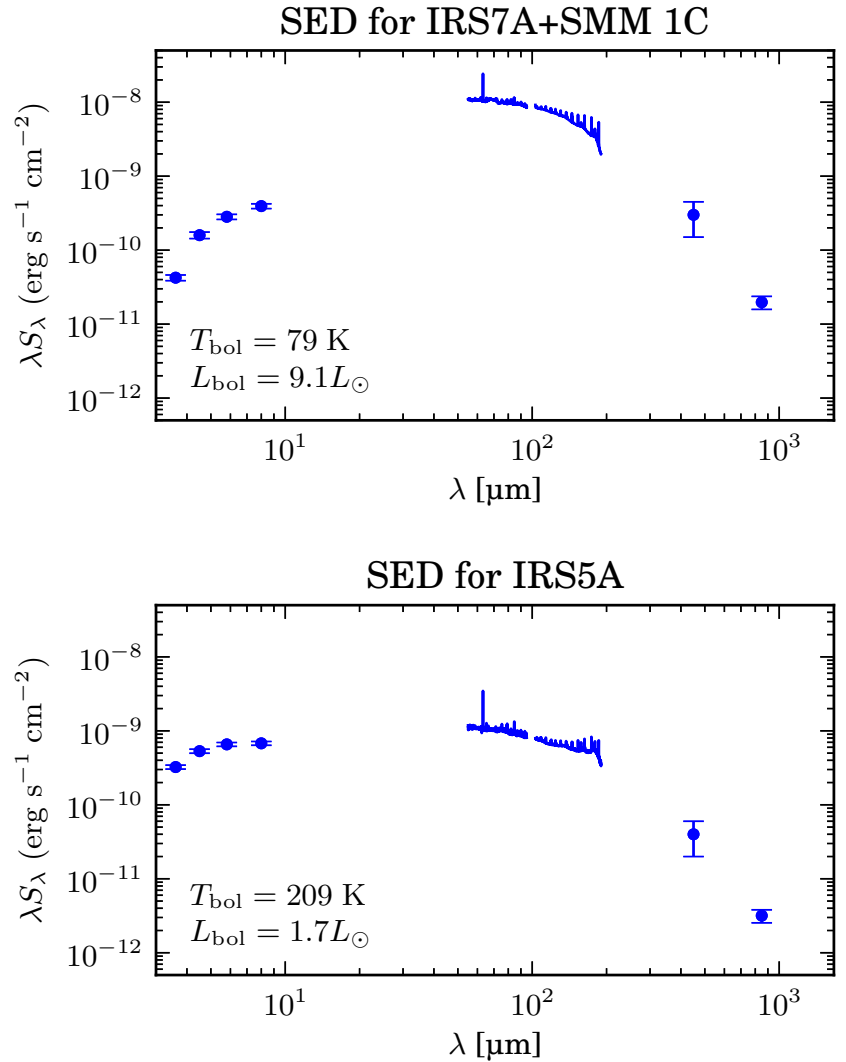
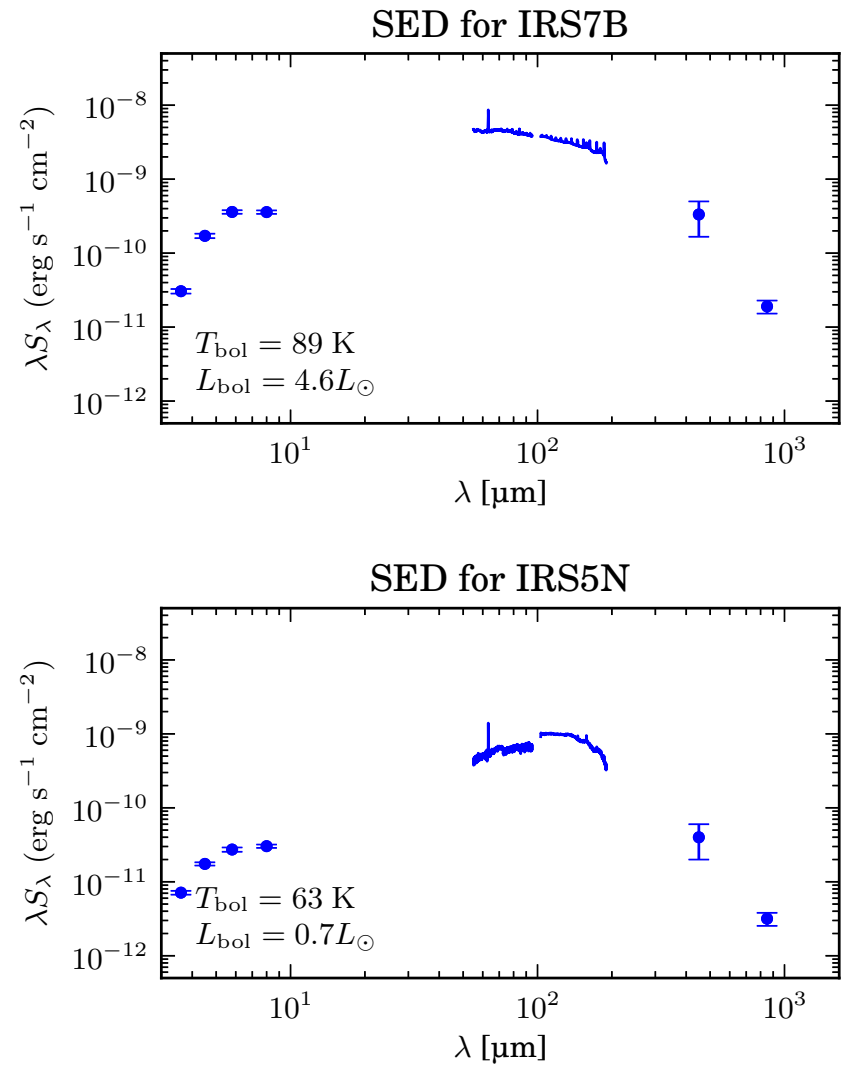

Fig. 8. Spectral energy distributions of the deconvolved point sources. The continuum data points come from Spitzer and SCUBA data. The spectra are deconvolved PACS spectra. See Table D.1 for details and references.

Table 4. Results of SED fits.

\begin{tabular}{lrrc}
\hline \hline Source & $\begin{array}{r}T_{\text {bol }} \\
{[\mathrm{K}]}\end{array}$ & $\begin{array}{r}L_{\text {bol }} \\
{\left[L_{\odot}\right]}\end{array}$ & $L_{\text {bol }} / L_{\text {submm }}$ \\
\hline IRS7A+SMM 1C & 79 & 9.1 & 99 \\
IRS7B & 89 & 4.6 & 48 \\
R CrA & 889 & 53.4 & $\ldots$ \\
IRS5A & 209 & 1.7 & 135 \\
IRS5N & 63 & 0.7 & 55 \\
\hline
\end{tabular}

bolometric temperature of SMM 1C is lower, and that of IRS7A is higher.

Differences between infrared and submillimetre continuum emission are also found between IRS5A and IRS5N in the IRS5 field. It has been suggested that IRS5A, because it is a binary $(\sim 100 \mathrm{AU})$, has all the dust in the disc cleared away (Jensen et al. 1996; Peterson et al. 2011), explaining why it is not detected by SMA mm observations. According to Peterson et al. (2011), both IRS5A and IRS5N are Class I sources or younger, with IRS5N having a steeper MIR spectral slope $\alpha$ than IRS5A. The observations of IRS5 can be used as a comparison for the IRS7 sources, since they should be less affected by the irradiation from R CrA. Although IRS5A has a MIR luminosity a few times higher than that of IRS7A and IRS7B, it shows only moderate line emission in the FIR (Herschel) data.

The SEDs of the residual structures Res N-c and Res S-c within the PACS band (Fig. 9) are found to peak at around the same wavelength as typical embedded YSOs do (such as the Class 0 source IRS5N). The SED peak around $100 \mu \mathrm{m}$ corresponds to a black-body dust temperature of $\sim 40 \mathrm{~K}$.

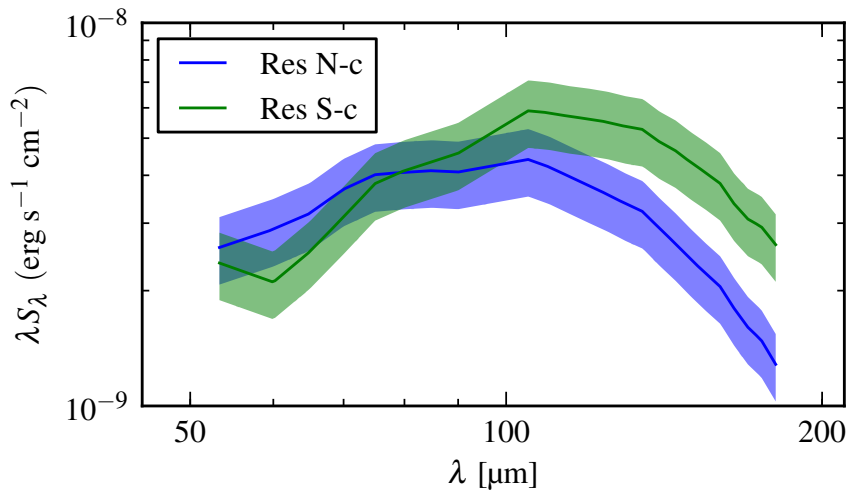

Fig. 9. Spectral energy distributions of the continuum residual regions Res N-c and Res S-c within the PACS band. The shading shows the $20 \%$ calibration uncertainty of the Herschel/PACS spectrometer.

\subsubsection{Spectral classification and SED of R CrA}

The WHT optical spectrum of R CrA is consistent with a photosphere of a star with spectral type B3-A0, after a comparison with the Pickles (1998) compilation of photospheric templates. The higher Balmer lines are seen in absorption and are used for the spectral comparison. The large range in acceptable spectral types is caused by the possibility of emission and absorption affecting the Balmer line equivalent widths. Strong emission is detected in $\mathrm{H} \alpha$ and $\mathrm{H} \beta$, and P-Cygni and inverse P-Cygni absorption are detected in some lines by Brown et al. (2013). Both emission and absorption may affect the equivalent widths in the photospheric lines. A high-resolution spectrum would be required to improve the spectral type. 


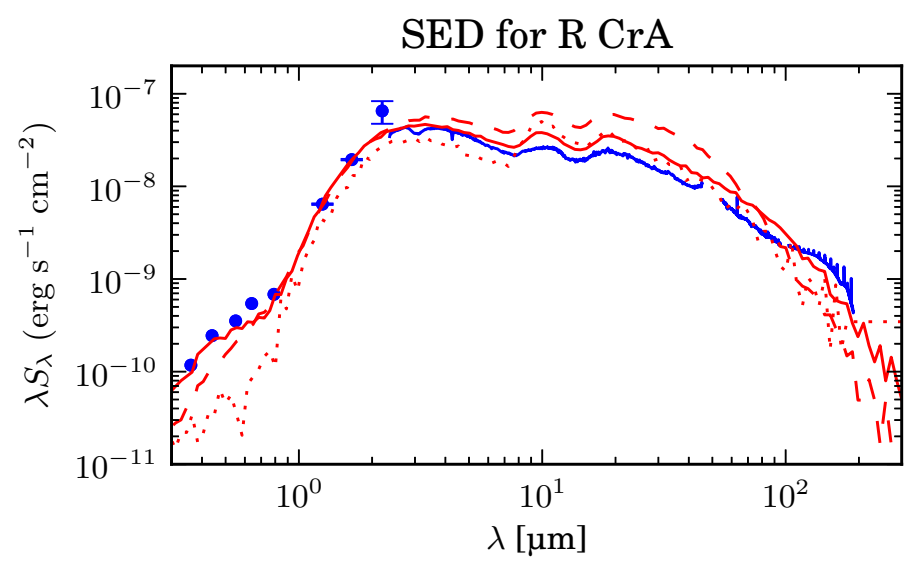

Fig. 10. The R CrA SED (blue) with optical (SAAO) and 2MASS data points, and ISO SWS and Herschel/PACS spectra. We note that this SED has a different $x$-axis than the SEDs in Fig. 8. Overplotted are the best, fifth best, and tenth best fits from the Robitaille model (red solid, dashed, and dotted). The best fit corresponds to Robitaille model ID 3011150 , with a disc observed at an inclination of $\sim 81^{\circ}$, an $A_{V}=3.27$, the distance $130 \mathrm{pc}$, and apertures similar to those of the instruments used for the actual observations. The sawtooth pattern at long wavelengths is present because the model is not very accurate at these wavelengths. The ten best fits all correspond to sources with luminosities of at least $480 L_{\odot}$ and high inclinations $\left(>80^{\circ}\right)$. For the PACS fit, the continuum flux densities at $70 \mu \mathrm{m}, 100 \mu \mathrm{m}$, and $160 \mu \mathrm{m}$ have been used.

This spectral type is consistent with the most reliable literature spectral types of B5 (Gray et al. 2006) and B8 (Bibo et al. 1992), which were also obtained using blue spectra. Other spectral types range from A0-F5 (e.g. Joy 1945; Greenstein \& Aller 1947; Hillenbrand et al. 1992; Vieira et al. 2003), but are typically based on red spectra, which are much less sensitive to the spectral type of hot stars. The spectrum cannot be well fit with the median interstellar extinction law, using a total-to-selective extinction ratio $R_{V}=3.1$. For the Weingartner \& Draine (2001) extinction law with $R_{V}=5.5$, the $A_{V}=4.5$ for an A0 spectral type and 5.3 for a B3 spectral type. A higher $A_{V}$ may be obtained with a higher $R_{V}$ (Manoj et al. 2006); however, such a high $R_{V}$ is not necessary to explain the shape of the optical spectrum.

The $V$ magnitude at the time of our observation was $\sim 13.2$ mag., as measured in our spectrum. The parameters B6 spectral type, $A_{V}=5.0$, and $d=130 \mathrm{pc}$ lead to a luminosity of $22 L_{\odot}$, which is much smaller than that inferred from the total SED and much smaller than that expected for a young B star. The $V$-band magnitude is variable by $\sim 3 \mathrm{mag}$. (Bibo et al. 1992). At its brightest, the star could be $350 L_{\odot}$, assuming no change in the measured extinction. Alternatively, the measured luminosity may be much lower than the real luminosity if the star is seen edge-on, as found in the Robitaille models (see below).

The SED of R CrA including SAAO, 2MASS, ISO SWS, and Herschel/PACS data points, is shown in Fig. 10 (blue data points and spectra). We find that R CrA has a bolometric temperature of $\sim 900 \mathrm{~K}$, making it a Class II YSO; and a luminosity of $53 L_{\odot}$, significantly lower than the previous value $99-166 L_{\odot}$ (Bibo et al. 1992; the lower value is from integration of the SED and the higher value is from a model of the extinction-free SED). Most of this discrepancy is present because Bibo et al. (1992) used KAO data for the FIR data points, and the large KAO beam included most of the R CrA cloud. As a result, the KAO $100 \mu \mathrm{m}$ flux is 7 times higher than our deconvolved value for R CrA measured with PACS. Another contribution to the large spread in the spectral classification data in the literature (F5 to B5) could be variability of the source (see e.g. Herbst \& Shevchenko 1999).

Here we attempt to constrain the physical properties of R CrA by the use of another method: using a database of SED models of YSOs (Robitaille et al. 2006) and an online ${ }^{3}$ fitting tool (Robitaille et al. 2007), we find that the observed SED can best be explained by a source with stellar mass $M \approx 6 M_{\odot}$, a stellar temperature corresponding to a B3 star, a total stellar luminosity $L \approx 900 L_{\odot}$, a nearly edge-on disc (inclination $80^{\circ}$ ), and an extinction of $A_{V} \approx 3.3$. The fit is found in Fig. 10, together with the fifth and tenth best fits from this model. The ten best fits all correspond to sources with $L_{\text {bol }} \gtrsim 480 L_{\odot}$ and nearly edge-on discs. We also investigate the model SED of a star with the same properties as the best fit, but with a face-on disc. It is found to have flux densities more than an order of magnitude higher in the UV/optical and a few times higher in the infrared/submm than the edge-on counterpart. Thus, assuming that $\mathrm{R} \mathrm{CrA}$ behaves like this model star, it will heat some parts of the surrounding regions much more efficiently than other parts, perhaps giving rise to the ridge-like structures of heated gas and dust. Differences in the density distribution could also contribute to the uneven temperatures in the region. Since the SED database is not exhaustive, and because of the large number of free parameters, the use of this model and the resulting interpretation could be unreliable. Lindberg \& Jørgensen (2012) estimate a minimum luminosity for R CrA of $100 L_{\odot}$ in order to heat the molecular gas to the measured temperatures.

\subsection{Rotational diagram analysis}

If the line emission is optically thin, the flux of a spectral line can be converted into a population of molecules in the upper state of the rotational transition it represents. Rotational temperatures and the total number of emitting molecules can be estimated by fitting a line to a plot of upper-state population versus upperstate energy (Goldsmith \& Langer 1999; Green et al. 2013). To evaluate the line flux of each spectral line for each YSO, one could either use the line flux in the spaxel closest to the YSO (with a wavelength-dependent correction factor ${ }^{4}$ applied), or the POMAC method described in Sect. 3 and Appendix C. When studying an isolated point source, the difference in the results of these two methods should be small, at least if the amount of extended emission is reasonably low. However, in a field with several YSOs (like IRS7), the emission from the YSOs would spill over into each other's spaxels, so that a strong emitter could influence the measured flux in a weaker nearby source. This spill-over contribution is minimised when the POMAC method is used. In this section, all rotational diagrams are calculated with fluxes estimated from the POMAC algorithm.

\subsubsection{CO - point-source emission}

In the non-deconvolved YSO spaxels, up to $28 \mathrm{CO}$ lines are detected, from the $J=13 \rightarrow 12$ line at $200 \mu \mathrm{m}$ to the $J=$ $40 \rightarrow 39$ line at $66 \mu \mathrm{m}$. However, some CO lines, including the $J=13 \rightarrow 12$ line, lie in the leakage spectral region (Green et al. 2013), and others are blended with other spectral lines (see Table 3). These lines will not be used in the rotational diagrams. We also detect five ${ }^{13} \mathrm{CO}$ lines $(J=14 \rightarrow 13$ to $J=21 \rightarrow 20$;

\footnotetext{
3 http://caravan.astro.wisc.edu/protostars/

4 Refer to the PACS manual v. 2.4, Fig. 4.5: http://herschel. esac. esa.int/Docs/PACS/html/pacs_om.html
} 
Table 5. CO rotational temperatures and total number of molecules.

\begin{tabular}{|c|c|c|c|c|}
\hline YSO & $\begin{array}{l}T_{\text {warr }}{ }^{a} \\
{[\mathrm{~K}]}\end{array}$ & $\begin{array}{l}\mathcal{N}_{\text {warm }}{ }^{a} \\
{\left[10^{48}\right]}\end{array}$ & $\begin{array}{l}T_{\text {hot }^{a}} \\
{[\mathrm{~K}]}\end{array}$ & $\begin{array}{l}\mathcal{N}_{\text {hot }}{ }^{a} \\
{\left[10^{48}\right]}\end{array}$ \\
\hline IRS7A+SMM 1C ${ }^{b}$ & $294 \pm 16$ & $33.0 \pm 3.0$ & $682 \pm 34$ & $2.7 \pm 0.3$ \\
\hline IRS7B & $273 \pm 14$ & $12.0 \pm 1.1$ & $710 \pm 54$ & $0.51 \pm 0.07$ \\
\hline $\mathrm{R} \mathrm{CrA}$ & $287 \pm 16$ & $5.4 \pm 0.5$ & $992 \pm 91$ & $0.27 \pm 0.03$ \\
\hline IRS5A & $293 \pm 17$ & $3.1 \pm 0.3$ & $1417 \pm 780$ & $0.12 \pm 0.04$ \\
\hline IRS5N & $283 \pm 24$ & $0.7 \pm 0.1$ & $\ldots$ & $\ldots$ \\
\hline Res SW-1 & $285_{-33}^{+15}$ & $5.0 \pm 0.5$ & $653_{-69}^{+68}$ & $0.35 \pm 0.06$ \\
\hline Res E-1 & $287_{-33}^{+16}$ & $1.7 \pm 0.2$ & $1015 \pm 198$ & $0.09 \pm 0.02$ \\
\hline Res N-1 & $281_{-32}^{+15}$ & $3.3 \pm 0.3$ & $898 \pm 334$ & $0.11 \pm 0.06$ \\
\hline Res W-1 & $253_{-26}^{+12}$ & $13.0 \pm 1.2$ & $751_{-94}^{+93}$ & $0.40 \pm 0.07$ \\
\hline CrA point-source average & $286 \pm 3^{c}$ & $10.8 \pm 5.2^{c}$ & $950 \pm 148^{c}$ & $0.90 \pm 0.52^{c}$ \\
\hline $\mathrm{CrA}$ extended average & $277 \pm 7^{c}$ & $5.8 \pm 2.2^{c}$ & $829 \pm 69^{c}$ & $0.24 \pm 0.07^{c}$ \\
\hline NGC 1333 IRAS 4B ${ }^{d}$ & 280 & 40 & 880 & 3 \\
\hline Serpens SMM1 ${ }^{e}$ & $337 \pm 40$ & $\ldots$ & $622 \pm 30$ & $\ldots$ \\
\hline Serpens SMM3/4 average ${ }^{f}$ & $260 \pm 10^{c}$ & $49 \pm 6^{c}$ & $800 \pm 60^{c}$ & $2.2 \pm 0.6^{c}$ \\
\hline DIGIT average ${ }^{g}$ & $355 \pm 3^{c}$ & $5.2 \pm 0.4^{c}$ & $814 \pm 29^{c}$ & $1.63 \pm 0.20^{c}$ \\
\hline HOPS average ${ }^{h}$ & $288 \pm 14^{c}$ & $\begin{array}{l}3.2 \pm 0.4 \\
\ldots\end{array}$ & $735 \pm 37^{c}$ & $\begin{array}{l}1.00 \pm 0.20 \\
\ldots\end{array}$ \\
\hline
\end{tabular}

Notes. ${ }^{(a)}$ The methods used for calculating the error estimates are discussed in Sects. 4.2.1 and 4.2.2. ${ }^{(b)}$ The separation between these sources is too small to allow treating their line fluxes independently using POMAC. ${ }^{(c)}$ For the sample averages, standard deviations of the mean (the standard deviation of the sample divided by the square root of the sample size) of the significant fits are given. ${ }^{(d)}$ From Herczeg et al. (2012). ${ }^{(e)}$ From Goicoechea et al. (2012). ${ }^{(f)}$ Average of SMM3b, SMM3c, SMM3r, and SMM4, from Dionatos et al. (2013), assuming a distance of 415 pc. ${ }^{(g)}$ From Green et al. (2013), including all DIGIT embedded sources except the CrA and Serpens SMM3/4 sources. The Serpens region is, like the CrA region, affected by confusion and is treated in a separate paper (Dionatos et al. 2013). ${ }^{(h)}$ From Manoj et al. (2013).

three of the eight lines in this range are blended with stronger spectral lines).

The rotational temperature found by this method will correspond to the kinetic temperature if the cloud is homogeneous and in LTE, and the spectral lines are optically thin. However, in the case of $\mathrm{CO}$, two rotational temperatures fit the data much better than does a single rotational temperature (see Green et al. 2013 for a justification of the use of a two-component fit; and also Manoj et al. 2013; Karska et al. 2013; Dionatos et al. 2013). In the case of $\mathrm{CrA}$, the warm component has a temperature of approximately $300 \mathrm{~K}$ for all point sources, whereas the hot component has a larger spread around 650-1400 K (mainly due to a lower $\mathrm{S} / \mathrm{N}$ ), which are both in the same order as in many other embedded YSOs (see e.g. Green et al. 2013; Manoj et al. 2013; Herczeg et al. 2012).

In Table 5, the calculated $\mathrm{CO}$ rotational temperatures and total number of molecules are given along with the same properties of other similar sources in the literature. The errors in the temperatures and numbers of molecules are calculated assuming a systematic error of $20 \%$ in the line fluxes; even though the line fluxes are from the same spectrum, the systematic errors are assumed to be independent. The $\mathrm{CO}$ rotational diagrams are found in Fig. 11.

The CO rotational temperatures towards IRS5A established from the POMAC fluxes are found to be within the errors of those derived from just using the central spaxel flux and the PSF correction factor, as expected for this relatively isolated point source. The $\mathrm{CO}$ emission of IRS5N is five times fainter than that of IRS5A, but has a similar CO rotational temperature in the warm component. No hot component is detected in IRS5N; if present at a ratio relative to the warm component seen in other sources, it would be below the detection limit.

We also perform a rotational diagram fit for the ${ }^{13} \mathrm{CO}$ data in IRS7A+SMM 1C (see Fig. 12). From the five detected lines, we get a rotational temperature of $266 \pm 35 \mathrm{~K}$, which is consistent with the ${ }^{12} \mathrm{CO}$ temperature $294 \pm 16 \mathrm{~K}$. The number of molecules is found to be $(8.90 \pm 1.58) \times 10^{47}$, but if the temperature is constrained to the ${ }^{12} \mathrm{CO}$ value the number of molecules becomes slightly lower, $7.47 \times 10^{47}$. With the ${ }^{13} \mathrm{CO}$ rotational temperature constrained to the ${ }^{12} \mathrm{CO}$ value, we find the ${ }^{12} \mathrm{CO} /{ }^{13} \mathrm{CO}$ abundance ratio to be $44 \pm 9$, corresponding to an optical depth of $0.56 \pm 0.24$ assuming the local ISM ${ }^{12} \mathrm{C} /{ }^{13} \mathrm{C}$ value of $77 \pm 7$ (Wilson \& Rood 1994). We adopt a CO line width of $\sim 7.5 \mathrm{~km} \mathrm{~s}^{-1}$ from Herschel HIFI observations of the ${ }^{12} \mathrm{CO}$ $J=16 \rightarrow 15$ line (Kristensen et al., in prep.). This value is comparable to the ${ }^{12} \mathrm{CO} J=7 \rightarrow 6$ quiescent component line width $\left(8 \mathrm{~km} \mathrm{~s}^{-1}\right.$; van Kempen et al. 2009a). We use RADEX (van der Tak et al. 2007), a non-LTE radiative transfer code for calculations of line strengths in isothermal homogeneous interstellar clouds, and find that this marginally optically thick result is consistent with a ${ }^{12} \mathrm{CO}$ column density of $\sim 10^{18} \mathrm{~cm}^{-2}$, corresponding to a size of the emitting region of the order of a few arcseconds ( $\sim 500 \mathrm{AU})$.

We have also investigated whether the data can be fitted with a single kinetic temperature component of much higher temperature and lower density, as suggested by Neufeld (2012). RADEX calculations assuming the same line width as above $\left(7.5 \mathrm{~km} \mathrm{~s}^{-1}\right)$ and the column density derived above $\left(\sim 10^{18} \mathrm{~cm}^{-2}\right)$ give a single kinetic temperature component with a best fit at $\sim 5000 \mathrm{~K}(>2500 \mathrm{~K}$ with a $1 \sigma$ certainty $)$ for an $\mathrm{H}_{2}$ density $(2.5 \pm 0.5) \times 10^{4} \mathrm{~cm}^{-3}$ for IRS7A+SMM 1C. For the best solution, the lowest- $J(J \lesssim 17)$ lines are marginally optically thick ( $\tau \sim 1$ ), while the lines with $J \gtrsim 22$ have optical depths $\tau \ll 1$. The reduced $\chi^{2}$ value for the best fit is 1.1 .

\subsection{2. $\mathrm{CO}$ - extended emission}

To investigate if the extended $\mathrm{CO}$ emission in the IRS7 region shows any variation in temperature, it was grouped into four 
$\mathrm{CO}$ rotational diagram of IRS7A+SMM 1C

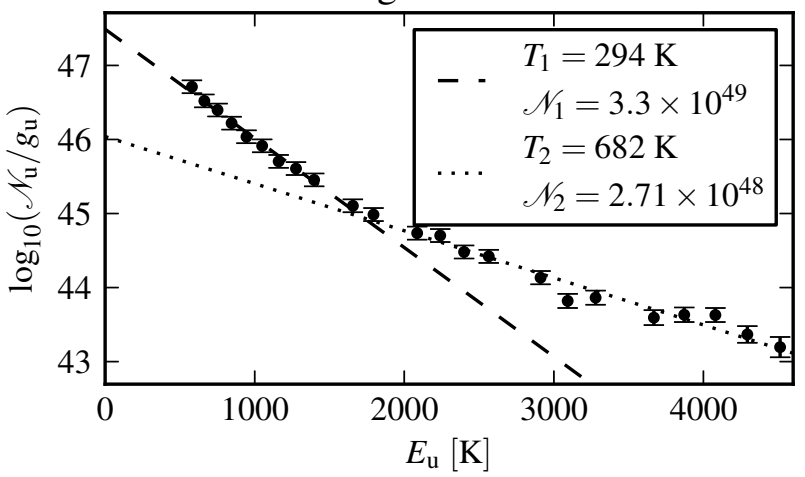

$\mathrm{CO}$ rotational diagram of $\mathrm{R} \mathrm{CrA}$

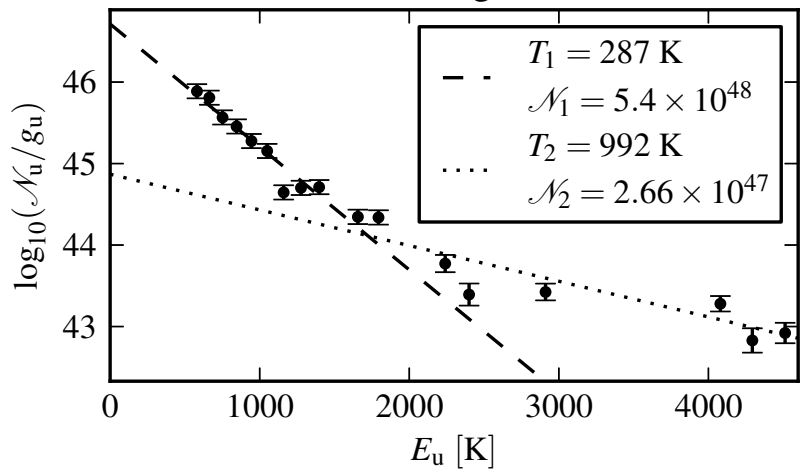

$\mathrm{CO}$ rotational diagram of IRS5N

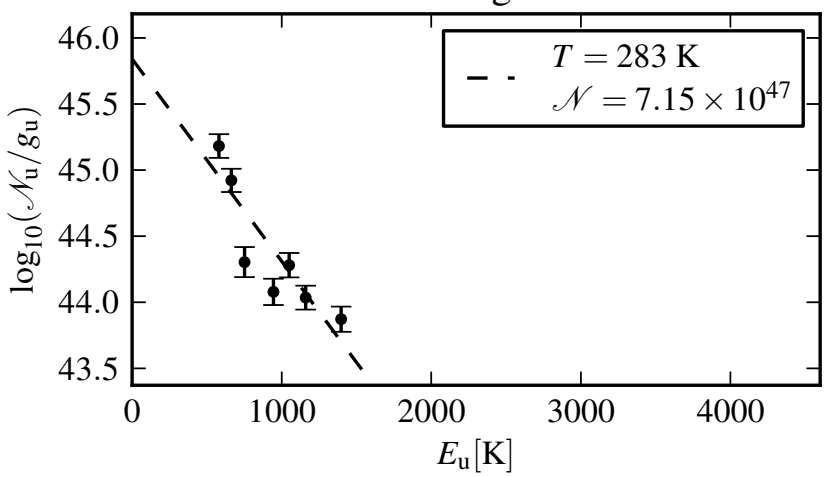

CO rotational diagram of IRS7B

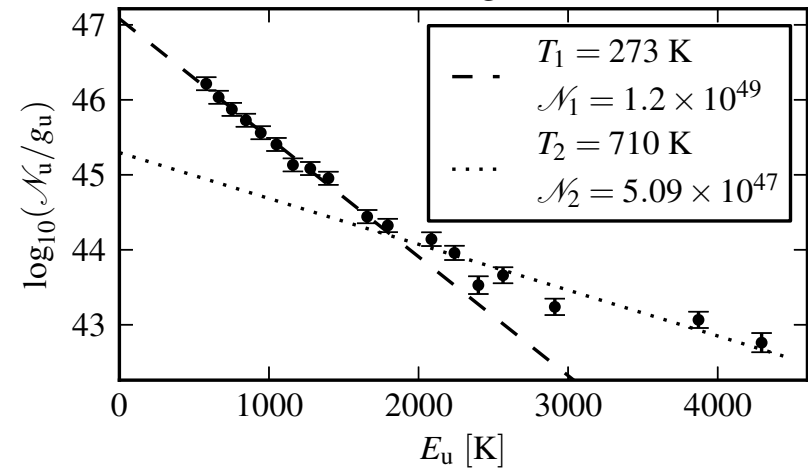

CO rotational diagram of IRS5A

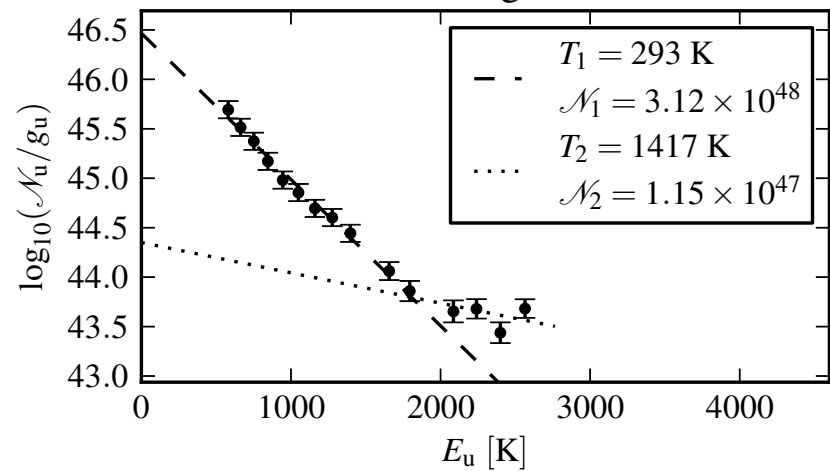

Fig. 11. CO rotational diagrams of the point sources in the deconvolved PACS data. Some high- $J$ data points are missing in some sources because many of these lines lie around the detection limit and the noise level fluctuates. $\mathcal{N}$ is the total number of CO molecules in each source given the rotational fit.

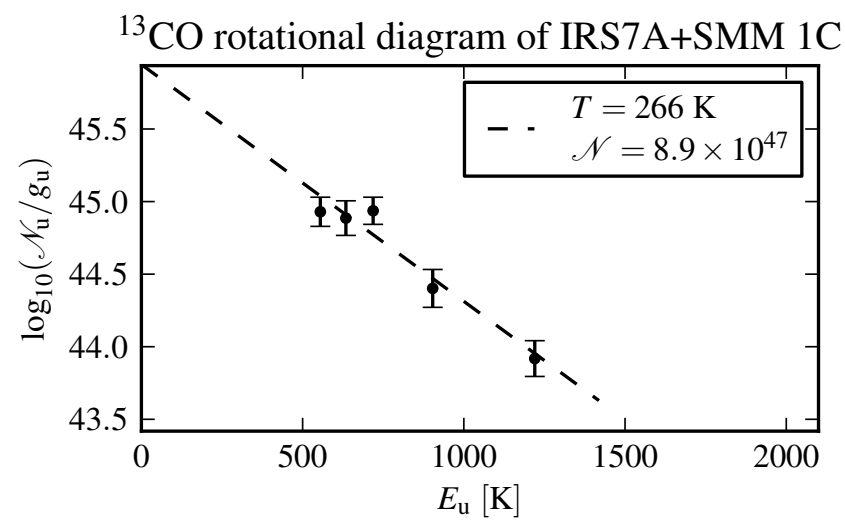

Fig. 12. ${ }^{13} \mathrm{CO}$ rotational diagram of IRS7A+SMM $1 \mathrm{C}$ from the deconvolved PACS data. $\mathcal{N}$ is the total number of ${ }^{13} \mathrm{CO}$ molecules in each source given the rotational fit. areas: southwest, east, northwest, and west of the YSOs (see Fig. 7). We call the four residual emission regions Res SW-1, Res E-1, Res N-1, and Res W-1 (1 for spectral line). Res N-1 overlaps with the central part of the continuum residual region Res N-c, and Res SW-l overlaps with the western part of Res S-c (see Fig. 6). By computing the residual spectral line emission in these regions we can produce rotational diagrams for this extended emission.

The resulting rotational diagrams are found in Fig. 13; as for the point-source emission, a warm and a hot component with rotational temperatures around $300 \mathrm{~K}$ and $900 \mathrm{~K}$, respectively, are found. The exact properties of the fits are included in Table 5. The rotational temperatures of the residual emission are within the errors of the point-source rotational temperatures.

On one hand, the extended emission is not deconvolved, and one can thus argue that a PSF correction factor needs to be applied to this data. On the other hand, since these fluxes are sums 
CO rotational diagram of Res SW-1

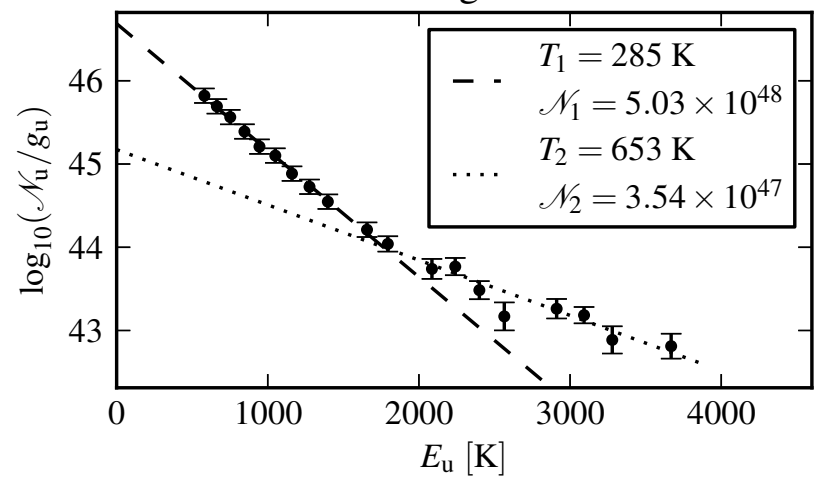

$\mathrm{CO}$ rotational diagram of Res $\mathrm{N}-1$

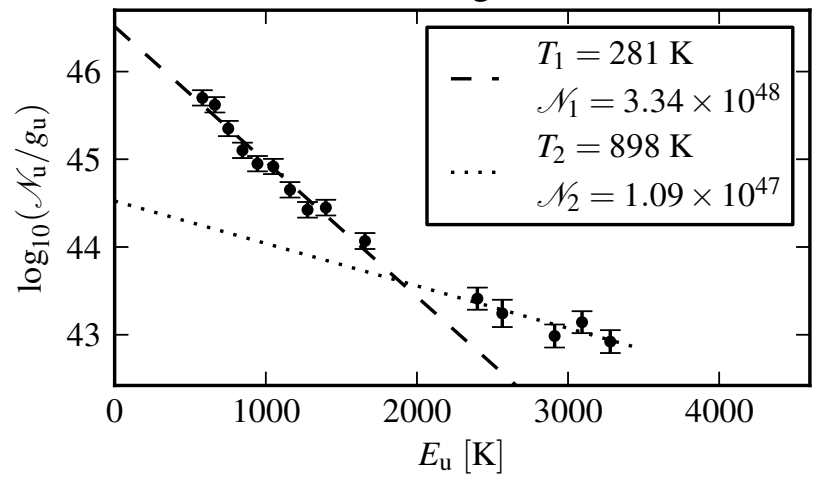

CO rotational diagram of Res E-1

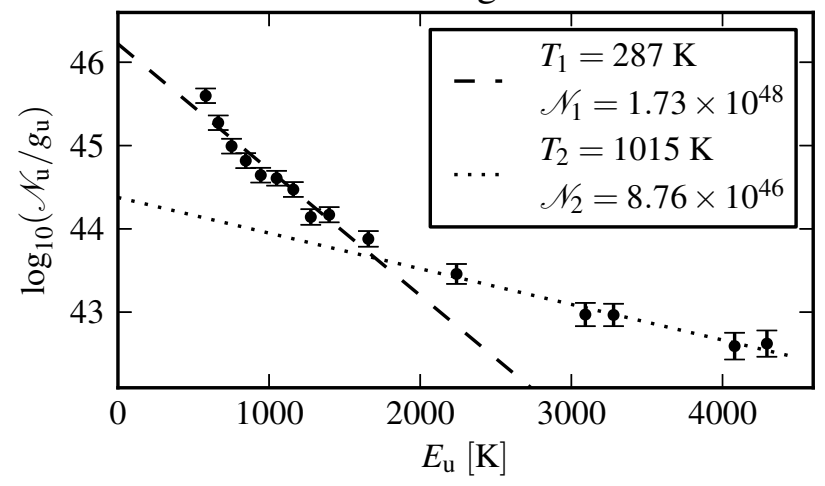

CO rotational diagram of Res W-1

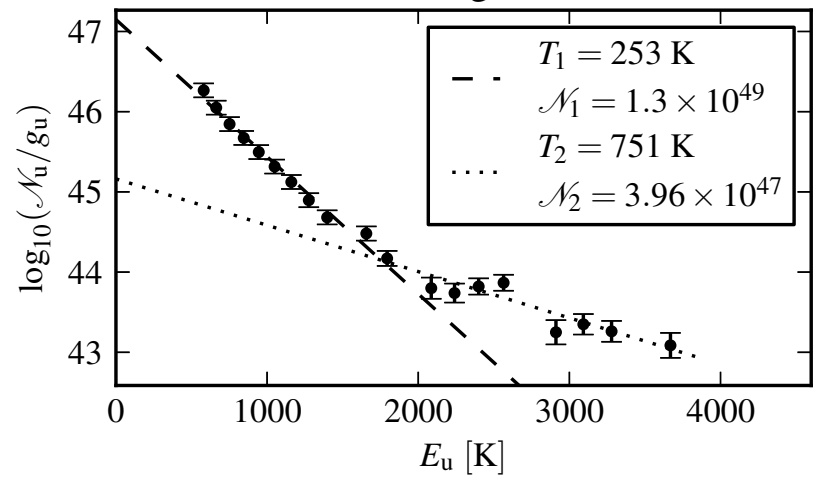

Fig. 13. CO rotational diagrams for the residual emission of the extended regions Res SW-1, Res E-1, Res N-1, and Res W-1. $\mathcal{N}$ is the total number of $\mathrm{CO}$ molecules in each source given the rotational fit.

of emission from several (2-6) spaxels (see Fig. 7), they are clearly in less need of PSF correction than point-source data. However, to take this issue into account, we calculate the rotational temperatures of the extended emission also using the PACS standard PSF correction factor from the PACS manual, and use the result to establish the lower boundary of the error estimate of these temperatures.

\subsection{3. $\mathrm{OH}$ and $\mathrm{H}_{2} \mathrm{O}$}

The excitation conditions of the related species $\mathrm{OH}$ and $\mathrm{H}_{2} \mathrm{O}$ are treated together in this section.

For the unresolved $\mathrm{OH}$ doublets, the sum of both lines is measured and divided by 2 . In the rotational diagram fits we exclude the same lines as Wampfler et al. (2013). These excluded lines are the $119 \mu \mathrm{m}$ doublet (which in similar sources is found to be an optically thick transition), the $84.4 \mu \mathrm{m}$ line (CO blend), and the $98 \mu \mathrm{m}$ and $55 \mu \mathrm{m}$ doublets (lines in leakage regions). They are plotted with open circles in the rotational diagrams.

The $\mathrm{OH}$ rotational diagrams of the point sources are found in Fig. 14, and those of the extended emission in Fig. 15. The derived parameters are listed in Table 6 , where they are also compared to some other embedded sources in the literature. The CrA sources have fairly uniform excitation temperatures, and do not differ significantly from the other sources in the literature. We also produce $\mathrm{OH}$ rotational diagrams of the four extended regions (Fig. 15). The $\mathrm{OH}$ temperatures in the extended emission are similar to the point sources within $3 \sigma$.

For $\mathrm{H}_{2} \mathrm{O}$, we assume an ortho-to-para ratio of 3 (Herczeg et al. 2012). This assumption is accounted for in the rotational diagrams (Figs. 16-17). The $\mathrm{H}_{2} \mathrm{O}$ rotational diagrams show comparably large spreads, which are mainly due to subthermal excitation effects and optical depth effects on some of the lines (Herczeg et al. 2012). The apparent shift between ortho and para lines might be caused by either of these effects, or by an orthoto-para ratio lower than 3 , but without more elaborate radiative transfer models it is impossible to distinguish between these scenarios. The derived rotational temperatures and total numbers of molecules are shown in Table 7. As in the case of OH, non-LTE radiative transfer models can be used to resolve the optical depth effects (Herczeg et al. 2012). Another method would be to exclude spectral lines suspected to be optically thick. The $\mathrm{H}_{2} \mathrm{O}$ excitation temperatures are higher than the $\mathrm{OH}$ temperatures. The higher $\mathrm{H}_{2} \mathrm{O}$ temperature in $\mathrm{R} \mathrm{CrA}$ than in the other sources is probably caused by a smaller number of detected lines, which enhances the temperature since the stronger lines are optically thick. The observed $\mathrm{H}_{2} \mathrm{O}$ temperatures do not vary significantly from the DIGIT average. As in the $\mathrm{CO}$ and $\mathrm{OH}$ cases, the $\mathrm{H}_{2} \mathrm{O}$ rotational temperatures are similar in extended emission and the point sources.

\subsubsection{Comparison of the rotational diagrams}

The rotational temperatures of the three molecules studied in the FIR data $\left(\mathrm{CO}, \mathrm{OH}\right.$, and $\left.\mathrm{H}_{2} \mathrm{O}\right)$ are all different (see Tables 5-7). There are, however, relatively small spreads among the warm $\mathrm{CO}$ and $\mathrm{OH}$ temperatures. The warm $\mathrm{CO}$ temperature average is significantly lower in the CrA point-source sample than in the DIGIT sample (Green et al. 2013), but in agreement with the HOPS (Herschel Orion Protostar Survey) sample (Manoj et al. 2013); whereas the hot CO is in agreement between the CrA and DIGIT samples, but higher than in the HOPS sample. The $\mathrm{OH}$ 

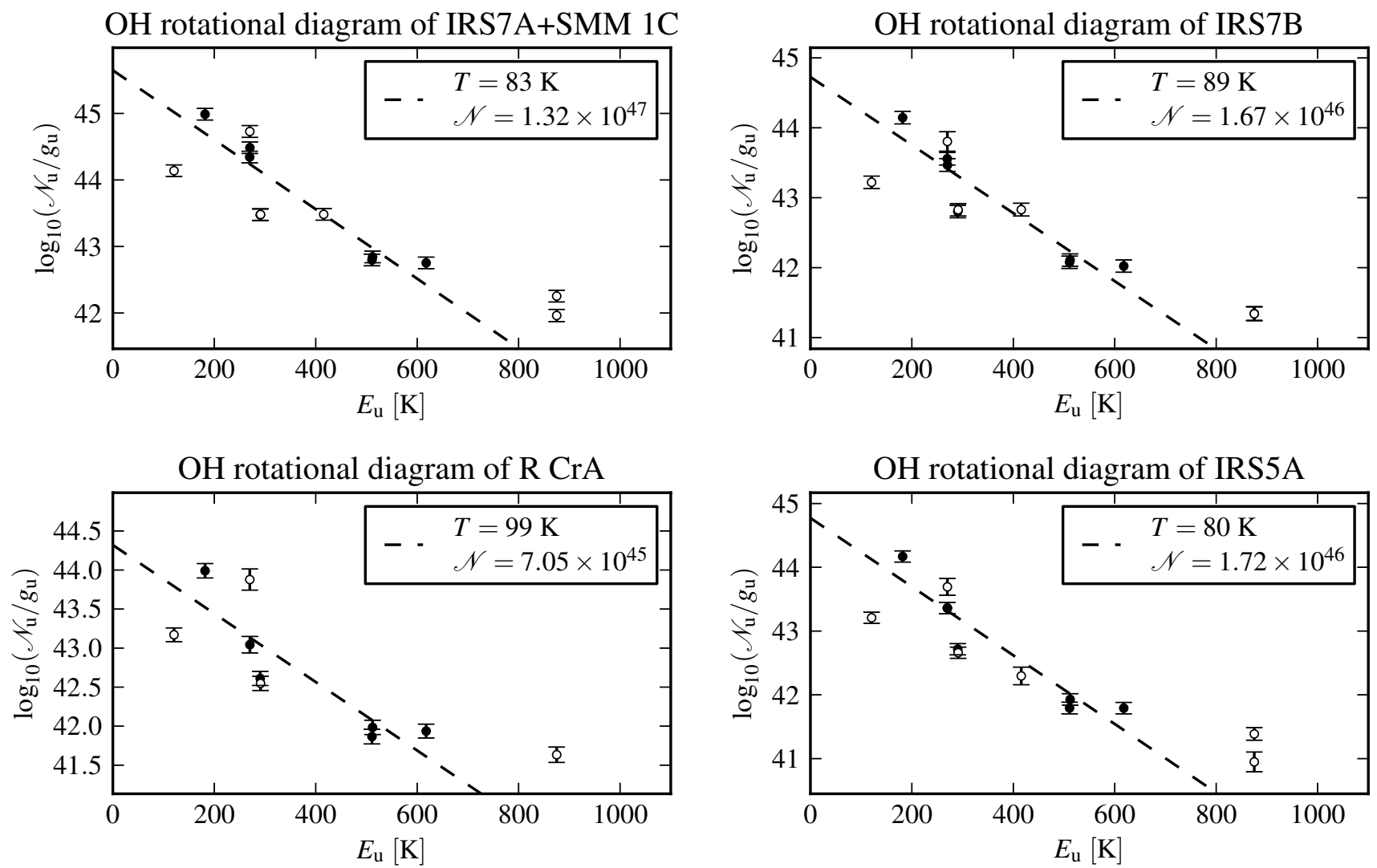

Fig. 14. OH rotational diagrams of the point sources in the deconvolved PACS data. The data points plotted as open circles were not included in the fit because of optical thickness, blends, or leakage. $\mathcal{N}$ is the total number of $\mathrm{OH}$ molecules in each source given the rotational fit.

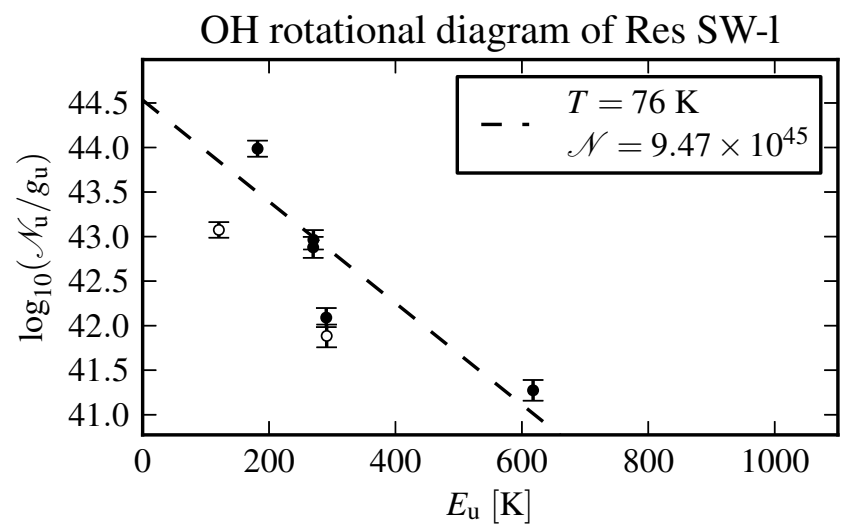

$\mathrm{OH}$ rotational diagram of Res N-1

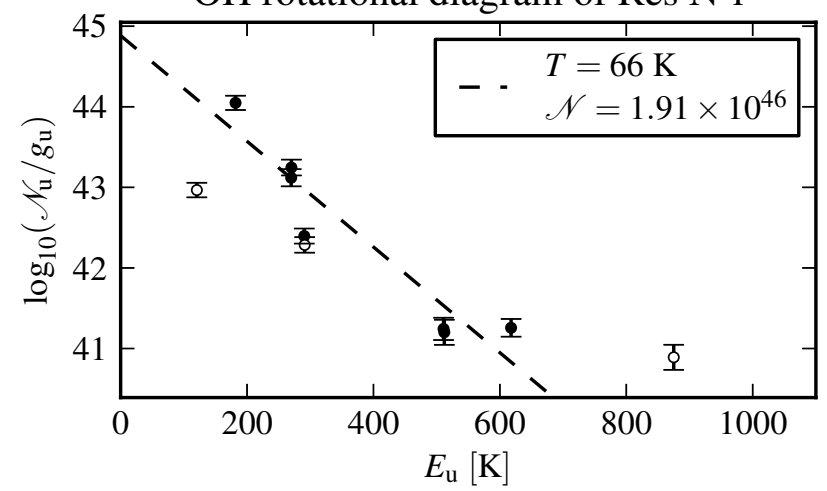

$\mathrm{OH}$ rotational diagram of Res E-1

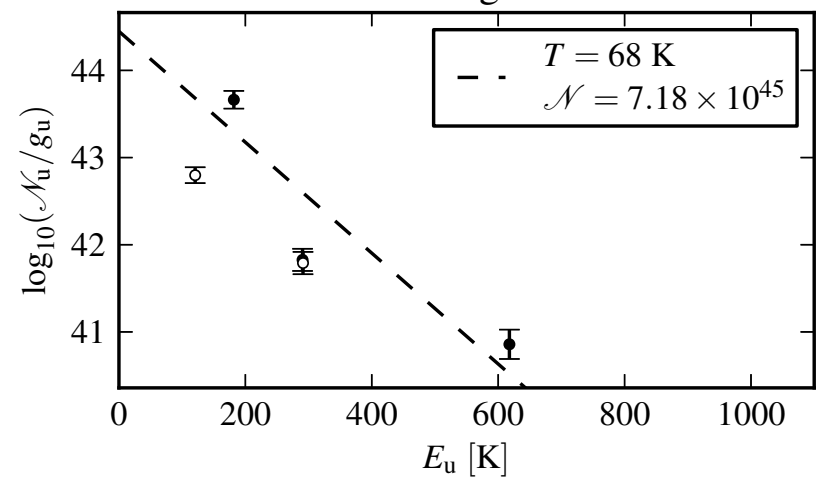

$\mathrm{OH}$ rotational diagram of Res W-1

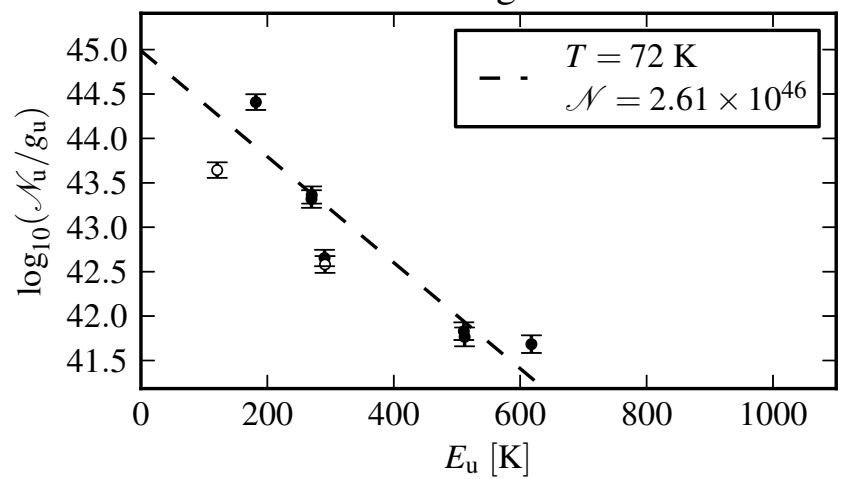

Fig. 15. OH rotational diagrams for the residual emission of the extended regions Res SW-1, Res N-1, Res E-1, and Res W-1. The data points plotted as open circles were not included in the fit because of optical thickness, blends, or leakage. $\mathcal{N}$ is the total number of OH molecules in each source given the rotational fit. 
$\mathrm{H}_{2} \mathrm{O}$ rotational diagram of IRS7A+SMM 1C

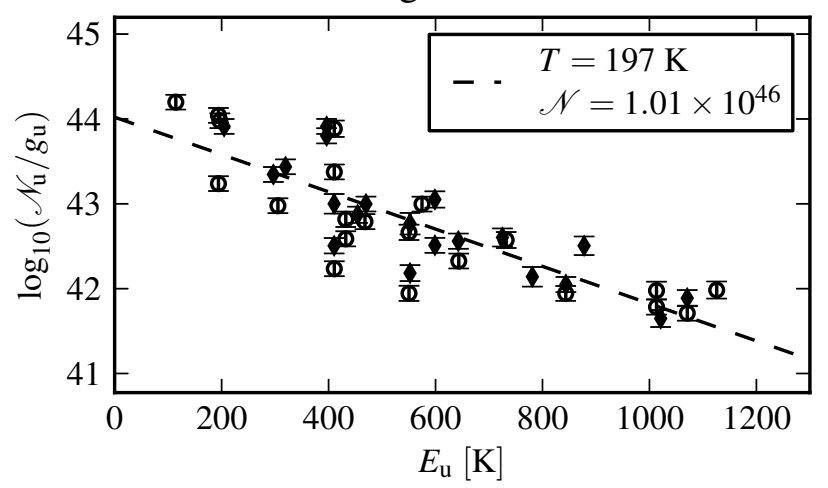

$\mathrm{H}_{2} \mathrm{O}$ rotational diagram of $\mathrm{R} \mathrm{CrA}$

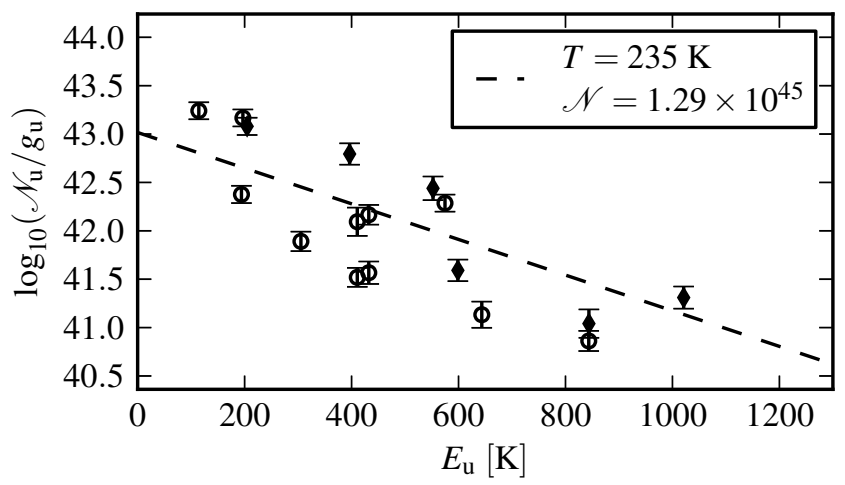

$\mathrm{H}_{2} \mathrm{O}$ rotational diagram of IRS5N

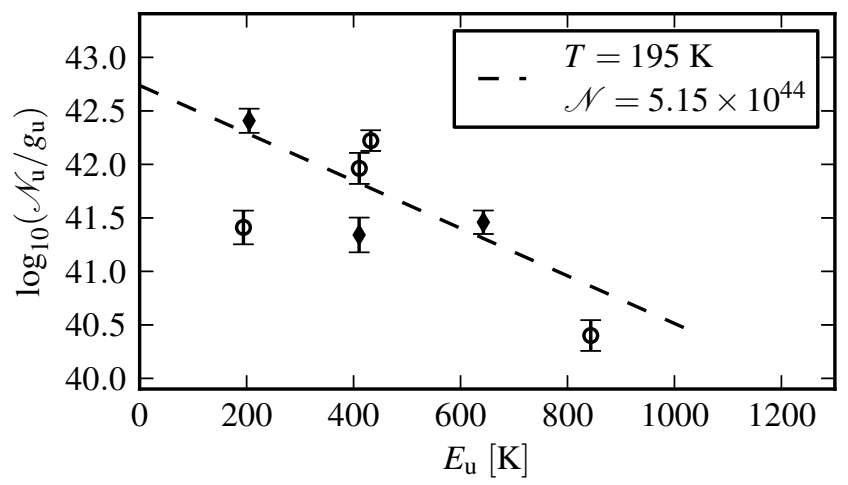

$\mathrm{H}_{2} \mathrm{O}$ rotational diagram of IRS7B

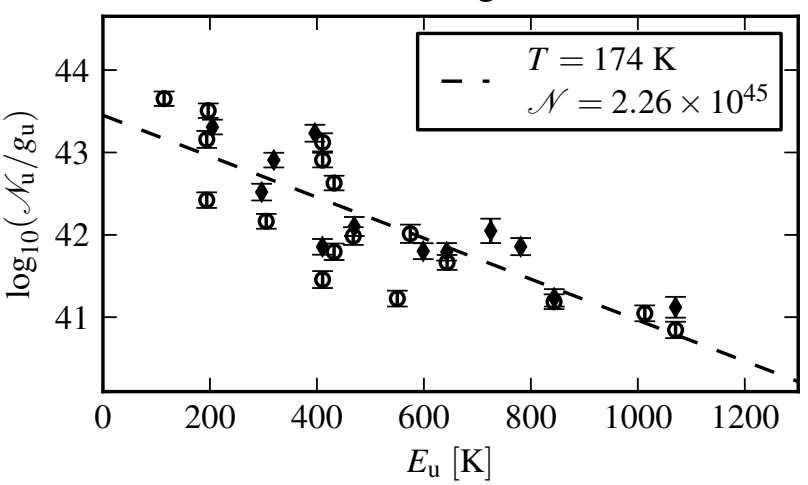

$\mathrm{H}_{2} \mathrm{O}$ rotational diagram of IRS5A

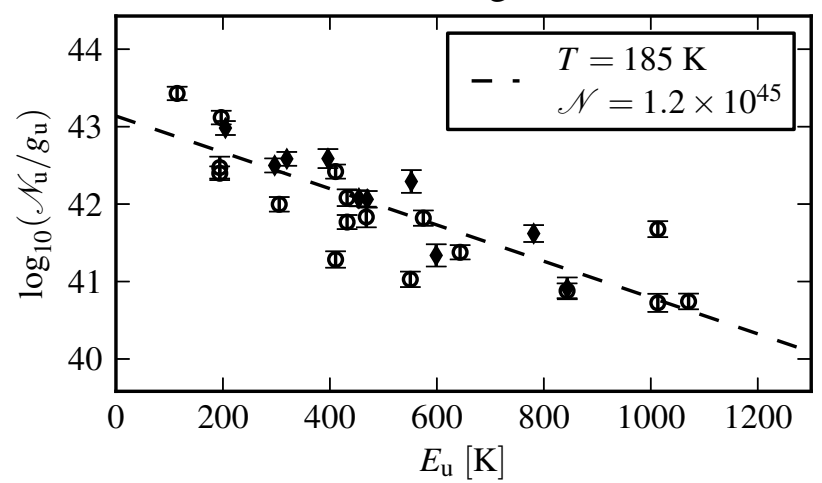

Fig. 16. $\mathrm{H}_{2} \mathrm{O}$ rotational diagrams of the point sources in the deconvolved PACS data. Ortho lines are marked with open circles and para lines with filled diamonds. $\mathcal{N}$ is the total number of $\mathrm{H}_{2} \mathrm{O}$ molecules in each source given the rotational fit.

and $\mathrm{H}_{2} \mathrm{O}$ temperatures are similar between the CrA and DIGIT samples, where the DIGIT sample average for $\mathrm{OH}$ has been recalculated for consistency using only the $\mathrm{OH}$ lines in our fits, and thus does not agree with the average value given by Green et al. (2013).

Comparing the number of molecules per source between the CrA and DIGIT samples (Green et al. 2013) shows that the average number of $\mathrm{CO}$ molecules is larger in the CrA sample, the average number of $\mathrm{OH}$ molecules is larger (but within errors) in the $\mathrm{CrA}$ sample, and the average number of $\mathrm{H}_{2} \mathrm{O}$ molecules is lower in the CrA sample. Comparing these results could however be biased, since it was not possible to construct $\mathrm{OH}$ and $\mathrm{H}_{2} \mathrm{O}$ rotational diagrams for all sources in the DIGIT sample where $\mathrm{CO}$ rotational diagrams could be made. Instead, calculations of line ratios in the whole DIGIT sample will be a better tracer of any difference in abundance ratios (see Sect. 5.4).

\section{Discussion}

\subsection{Survey of source properties}

In Table 8, some important properties of the studied point sources and extended line emission regions are tabulated along with the DIGIT sample averages. The properties of the sources in the CrA sample are found to be fairly typical for low-mass embedded protostars. Since IRS7A and SMM 1C cannot be separated in the PACS data it is difficult to draw any conclusions about the classes of the separate sources. However, their different appearance in continuum data of other bands (IRS7A is detected in MIR but not mm; SMM 1C is detected in mm but not MIR) and their combined $T_{\text {bol }}=80 \mathrm{~K}$ point towards SMM 1C being a Class 0 source; IRS5N is definitely a Class 0 object, but the other low-mass sources in the sample cannot consistently be assigned to Class 0 or Class I. 

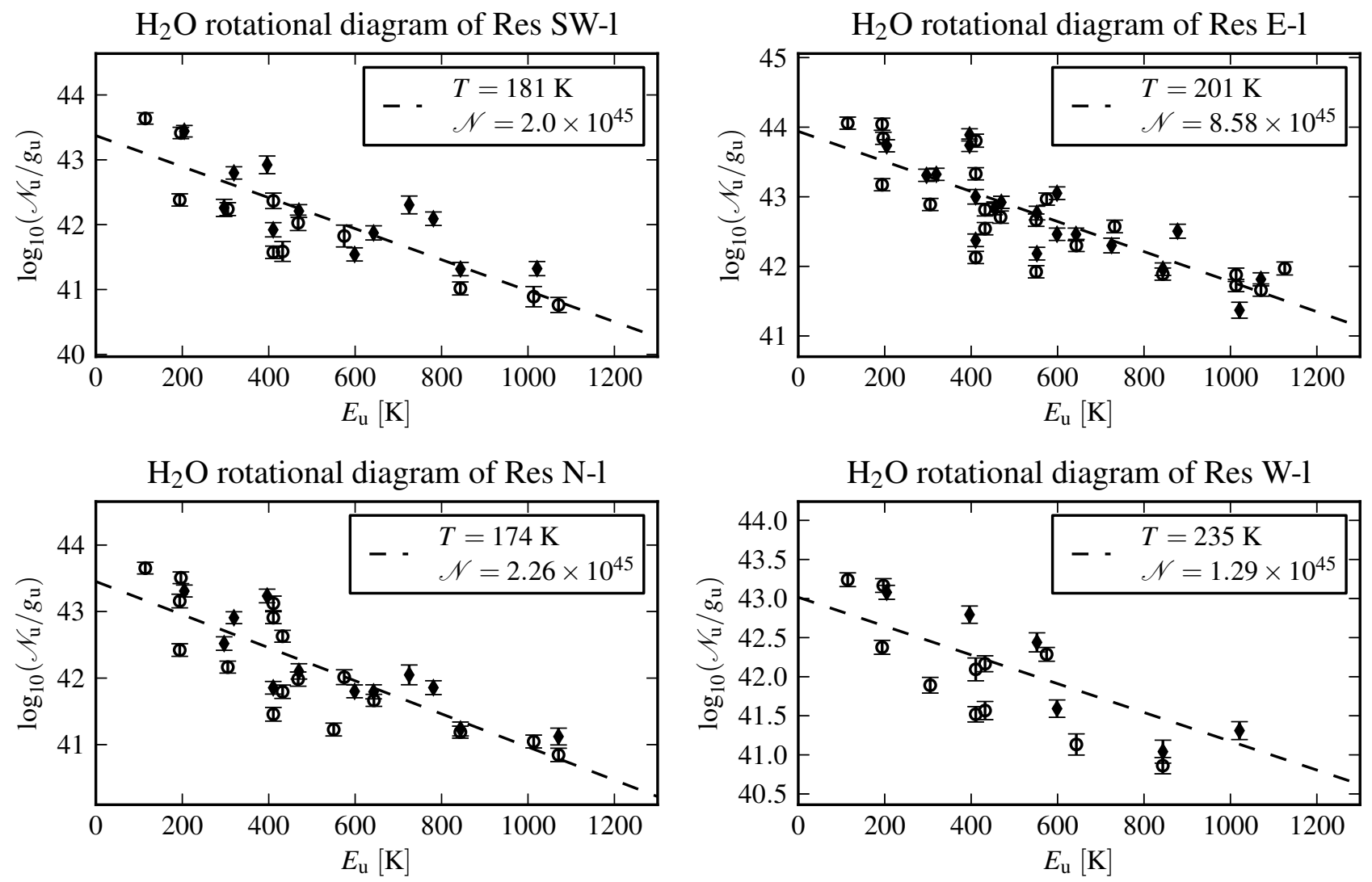

Fig. 17. $\mathrm{H}_{2} \mathrm{O}$ rotational diagrams for the residual emission of the extended regions Res SW-1, Res E-1, Res N-1, and Res W-1. Ortho lines are marked with open circles and para lines with filled diamonds. $\mathcal{N}$ is the total number of $\mathrm{H}_{2} \mathrm{O}$ molecules in each source given the rotational fit.

The SEDs of the extended continuum emission (in Res N-c and Res S-c; see Fig. 9) are similar to that of the Class 0 source IRS5N, which indicates that this gas has similar temperatures to those of very young protostellar cores. The dust black-body temperature of the two continuum ridges is found to be $40-50 \mathrm{~K}$, which is consistent with the $\mathrm{H}_{2} \mathrm{CO}$ temperature, estimated to be 40-60 K in Lindberg \& Jørgensen (2012). Lindberg \& Jørgensen (2012) showed that these temperatures cannot be caused by radiation from the low-mass protostars, but can instead be explained by external irradiation from $\mathrm{R} \mathrm{CrA}$.

\subsection{Excitation conditions}

As pointed out earlier, the $\mathrm{CO}$ excitation temperatures in the point sources are in good agreement with those found in larger samples of low-mass embedded objects (Green et al. 2013; Karska et al. 2013; Manoj et al. 2013). One possible explanation for the excitation conditions being similar towards the externally irradiated protostars and in sources not subject to external irradiation is that the irradiation from $\mathrm{R} \mathrm{CrA}$ is not substantial enough to dramatically change the properties of the high-temperature gas. The excitation conditions of the extended molecular line emission found across the IRS7 field are consistent with those of the compact objects, and do not change significantly across the IRS7 field. The appearance of this extended emission is, however, unusual. The excitation diagrams could be explained by a single-temperature non-LTE fit (Neufeld 2012), assuming a low gas density $\left(n \sim 10^{4} \mathrm{~cm}^{-3}\right)$, and that the gas is collisionally excited to high temperatures $(T \sim 5000 \mathrm{~K})$. However, the observation of strong and extended $\mathrm{H}_{2} \mathrm{CO}$ and $\mathrm{CH}_{3} \mathrm{OH}$ emission in the field makes this low-density scenario unlikely. Furthermore,
$\mathrm{H}_{2}$ densities $\lesssim 10^{6} \mathrm{~cm}^{-3}$ do not fit with the $\mathrm{H}_{2} \mathrm{CO}$ optical depth derived by Lindberg \& Jørgensen (2012). Another possibility is that the molecular gas towards the point sources and the extended gas are excited by different excitation mechanisms.

\subsection{Comparing FIR and $\mathrm{mm}$ spectral line data}

Strong CO $J=6 \rightarrow 5$ and $J=7 \rightarrow 6$ emission found on an east-west line centred at IRS7A (van Kempen et al. 2009a) are consistent with the residual CO regions Res E-1 and Res W-1, although the FIR data is more dominated by the point-source emission. The major difference between the morphology of the $\mathrm{H}_{2} \mathrm{CO}$ and $\mathrm{CH}_{3} \mathrm{OH} \mathrm{mm}$ line data (Lindberg \& Jørgensen 2012) and the FIR (Herschel) line data is that most of the FIR line emission is well-aligned with the mm and MIR continuum point sources, while the mm lines appear in more extended structures, which are not centred on these point sources. However, the residual PACS continuum emission after deconvolution of the pointsource emission (corresponding to extended dust continuum emission) shows shapes very similar to the high-temperature $\mathrm{H}_{2} \mathrm{CO}$ ridges observed in the mm (see Fig. 6). The $\mathrm{H}_{2} \mathrm{CO}$ rotational temperatures measured in the SMA/APEX mm data range from $30 \mathrm{~K}$ to $100 \mathrm{~K}$, but non-LTE modelling shows that the physical temperatures probably are of the order of 40-60 K (Lindberg $\&$ Jørgensen 2012). The PACS SEDs of these ridges show blackbody temperatures ( 40-50 K; see Fig. 9) consistent with the $\mathrm{H}_{2} \mathrm{CO}$ temperatures measured. The $\mathrm{H}_{2} \mathrm{CO}$ ridges are observed on relatively large scales ( $~ 8000 \mathrm{AU})$, and are not associated with the point sources.

The POMAC algorithm shows that most of the FIR molecular line emission originates from the MIR/(sub)mm point sources 
J. E. Lindberg et al.: Warm gas towards young stellar objects in Corona Australis

Table 6. $\mathrm{OH}$ rotational temperatures and total number of molecules.

\begin{tabular}{llc}
\hline \hline YSO & $T^{a}$ & \multicolumn{2}{l}{$\mathcal{N}^{a}$} \\
& {$[\mathrm{~K}]$} & {$\left[10^{45}\right]$} \\
\hline IRS7A+SMM 1C & $83 \pm 3$ & $132 \pm 12$ \\
IRS7B & $89 \pm 4$ & $16.7 \pm 1.5$ \\
R CrA & $99 \pm 5$ & $7.1 \pm 0.7$ \\
IRS5A & $80 \pm 3$ & $17.2 \pm 1.5$ \\
\hline Res SW-1 & $76 \pm 4$ & $9.5 \pm 1.1$ \\
Res E-1 & $68 \pm 4$ & $7.2 \pm 1.2$ \\
Res N-1 & $66 \pm 2$ & $19.1 \pm 2.1$ \\
Res W-1 & $72 \pm 2$ & $26.1 \pm 2.5$ \\
\hline CrA point-source average & $88 \pm 4^{b}$ & $43.3 \pm 25.7^{b}$ \\
CrA extended average & $71 \pm 2^{b}$ & $15.5 \pm 3.8^{b}$ \\
\hline NGC 1333 IRAS 4B $^{c}$ & 60 & 130 \\
Serpens SMM1 & $72 \pm 8$ & $\ldots$ \\
Serpens SMM3/4 average & $88 \pm 2^{b}$ & $26 \pm 3^{b}$ \\
\hline DIGIT $^{f}$ & $83 \pm 3^{b}$ & $24 \pm 3^{b}$ \\
\hline
\end{tabular}

Notes. ${ }^{(a)}$ The methods used for calculating the error estimates are the same as for the CO rotational diagrams, see Sects. 4.2.1 and 4.2.2. (b) For the sample averages, standard deviations of the mean of the significant fits are given (see Table 5). ${ }^{(c)}$ From Herczeg et al. (2012). Only the cool component based chiefly on PACS data is given here. (d) From Wampfler et al. (2013). ${ }^{(e)}$ Average of SMM3b and SMM4, from Dionatos et al. (2013), assuming a distance of $415 \mathrm{pc}$. This value is calculated excluding the same lines that have been excluded in this paper, and does not agree with the values given in Dionatos et al. (2013) for this reason. ${ }^{(f)}$ Average of the DIGIT sample, from Green et al. (2013), not including the CrA and Serpens sources. This value is calculated excluding the same lines that have been excluded in this paper, and for this reason does not agree with the value given in Green et al. (2013) (183 K).

IRS7A, SMM 1C, IRS7B, R CrA, and IRS5A. This emission is similar in excitation to what is found towards sources that are not subject to external irradation. However, through the deconvolution we also find extended line emission in the IRS7 region. Interestingly, the $\mathrm{CO}, \mathrm{OH}$, and $\mathrm{H}_{2} \mathrm{O}$ excitation conditions of the extended emission resemble those near the protostars (see Tables 5-7), and suggest that the temperatures are high, also on these relatively large scales $(>1000 \mathrm{AU}$ from the protostars). Such extended line emission, in particular the hot CO and $\mathrm{OH}$ emission, is unusual around low-mass embedded objects (see e.g. van Kempen et al. 2010; Green et al. 2013; Karska et al. 2013).

Still, the $\mathrm{H}_{2} \mathrm{CO}$ emission detected by SMA/APEX is even more extended, and not associated with the point sources. Regardless of the lower spatial resolution of the PACS data, it is certain that the FIR and mm line emission have different origins.

\subsection{Water and oxygen chemistry}

Assuming that the region around R CrA exhibits PDR-like conditions, the $\mathrm{OH}$ abundance should be enhanced with respect to the $\mathrm{H}_{2} \mathrm{O}$ abundance (see e.g. Walsh et al. 2013). We thus want to establish whether the $\mathrm{OH} / \mathrm{H}_{2} \mathrm{O}$ ratio is enhanced in these sources compared to other sources in similar stages of evolution. A firstorder comparison can be made by analysing the ratios of certain $\mathrm{OH}$ and $\mathrm{H}_{2} \mathrm{O}$ spectral lines in the DIGIT sample of embedded protostars (Green et al. 2013). So far, no systematic study of $\mathrm{OH}$ and $\mathrm{H}_{2} \mathrm{O}$ excitation diagrams and abundances in low-mass embedded objects is available. To avoid biases introduced by
Table 7. $\mathrm{H}_{2} \mathrm{O}$ rotational temperatures and total number of molecules.

\begin{tabular}{llc}
\hline \hline YSO & \multicolumn{1}{l}{$T^{a}$} & $\mathcal{N}^{a}$ \\
& {$[\mathrm{~K}]$} & {$\left[10^{45}\right]$} \\
\hline IRS7A+SMM 1C & $197 \pm 4$ & $10.1 \pm 0.3$ \\
IRS7B & $174 \pm 4$ & $2.3 \pm 0.1$ \\
R CrA & $235 \pm 9$ & $1.3 \pm 0.1$ \\
IRS5A & $185 \pm 5$ & $1.2 \pm 0.1$ \\
IRS5N & $195 \pm 20$ & $0.5 \pm 0.1$ \\
\hline Res SW-1 & $181 \pm 5$ & $2.0 \pm 0.1$ \\
Res E-1 & $201 \pm 4$ & $8.6 \pm 0.3$ \\
Res N-1 & $174 \pm 4$ & $2.3 \pm 0.1$ \\
Res W-1 & $235 \pm 9$ & $1.3 \pm 0.1$ \\
\hline CrA point-source average & $197 \pm 9^{b}$ & $3.1 \pm 1.6^{b}$ \\
CrA extended average & $198 \pm 12^{b}$ & $3.5 \pm 1.5^{b}$ \\
\hline NGC 1333 IRAS 4B $^{c}$ & $110 / 220$ & 100 \\
Serpens SMM1 & $136 \pm 27$ & $\ldots$ \\
Serpens SMM3/4 average $^{e}$ & $105 \pm 6^{b}$ & $25 \pm 3^{b}$ \\
\hline DIGIT $^{f}$ & $194 \pm 20^{b}$ & $7.7 \pm 2.6^{b}$ \\
\hline
\end{tabular}

Notes. ${ }^{(a)}$ The methods used for calculating the error estimates are the same as for the CO rotational diagrams, see Sects. 4.2.1 and 4.2.2. (b) For the sample averages, standard deviations of the mean of the significant fits are given (see Table 5). ${ }^{(c)}$ From Herczeg et al. (2012). Two separate cool and warm components. ${ }^{(d)}$ From Goicoechea et al. (2012). ${ }^{(e)}$ Average of SMM3b, SMM3c, SMM3r, and SMM4, from Dionatos et al. (2013), assuming a distance of 415 pc. ${ }^{(f)}$ Average of the DIGIT sample, from Green et al. (2013), not including the CrA and Serpens sources.

different amounts of detected lines and different methods of extracting the abundances, we instead compare the ratios of individual spectral line luminosities.

We need to compare $\mathrm{OH}$ and $\mathrm{H}_{2} \mathrm{O}$ lines thought to be optically thin, that are detected in many sources, that have small PSFs (short wavelengths) or at least similar PSFs (similar wavelengths), and that have similar upper-level energies to remove any bias. Thus, in the first four panels of Fig. 18, we plot four different line ratios of three $\mathrm{OH}$ lines and three $\mathrm{H}_{2} \mathrm{O}$ lines. The wavelengths differ by less than $20 \mu \mathrm{m}$ and the upper level energies by less than $80 \mathrm{~K}$ for each of the ratios. Details on the transitions are found in the figure. We find that all the CrA sources have an $\mathrm{OH} / \mathrm{H}_{2} \mathrm{O}$ line ratio higher than most other DIGIT embedded objects, which is indicative of PDR activity (Hollenbach $\&$ Tielens 1997). Also the extended regions have relatively high $\mathrm{OH} / \mathrm{H}_{2} \mathrm{O}$ ratios. We have also compared the $\mathrm{OH}$ (cf. Fig. 9 in Wampfler et al. 2013), $\mathrm{H}_{2} \mathrm{O}$, and $\mathrm{CO}(J=16 \rightarrow 15$; cf. Fig. 22 in Green et al. 2013) line luminosities to the bolometric luminosities of the DIGIT and WISH embedded sources, and found that the sources in CrA fall within the scatter around the linear correlation for all three lines, although on the higher end in the $\mathrm{OH}$ and $\mathrm{CO}$ cases. We find that the enhanced $\mathrm{OH} / \mathrm{H}_{2} \mathrm{O}$ ratio is mainly due to an increase in the $\mathrm{OH}$ flux rather than a decrease in the $\mathrm{H}_{2} \mathrm{O}$ flux.

From the dissociation of $\mathrm{OH}, \mathrm{O}$ should be a major destruction product, so we would also expect an enhancement of the [OI] line strengths compared to $\mathrm{H}_{2} \mathrm{O}$ and $\mathrm{OH}$ in a PDR (Hollenbach \& Tielens 1997). In the third row of diagrams in Fig. 18, we compare the line ratios of the [O I] $63.2 \mu \mathrm{m}$ line to one $\mathrm{H}_{2} \mathrm{O}$ line and one $\mathrm{OH}$ line with similar wavelengths and upper-level energies. We see a very strong enhancement of [O I], in particular in the extended regions, supporting the hypothesis of a PDR induced by external irradiation. 
Table 8. Properties of the studied point sources.

\begin{tabular}{|c|c|c|c|c|c|c|c|c|c|c|c|}
\hline \multirow[t]{2}{*}{ YSO } & \multicolumn{2}{|c|}{$\mathrm{CO}^{a}$} & \multicolumn{2}{|c|}{$\mathrm{OH}$} & \multicolumn{2}{|c|}{$\mathrm{H}_{2} \mathrm{O}$} & \multirow[b]{2}{*}{$\begin{array}{l}T_{\text {bol }} \\
{[\mathrm{K}]}\end{array}$} & \multirow[b]{2}{*}{$\begin{array}{l}L_{\mathrm{bol}} \\
{\left[L_{\odot}\right]}\end{array}$} & \multirow{2}{*}{$\begin{array}{l}\mathrm{MIR} / \mathrm{FIR} / \mathrm{mm} \\
\text { continuum } \\
\text { detected }\end{array}$} & \multirow{2}{*}{$\begin{array}{l}\mathrm{FIR} / \mathrm{mm} \\
\text { lines } \\
\text { detected }\end{array}$} & \multirow[t]{2}{*}{ Class } \\
\hline & $\begin{array}{l}T_{\text {rot }} \\
{[\mathrm{K}]}\end{array}$ & $\begin{array}{l}\mathcal{N} \\
{\left[10^{48}\right]}\end{array}$ & $\begin{array}{l}T_{\text {rot }} \\
{[\mathrm{K}]}\end{array}$ & $\begin{array}{l}\mathcal{N} \\
{\left[10^{45}\right]}\end{array}$ & $\begin{array}{l}T_{\text {rot }} \\
{[\mathrm{K}]}\end{array}$ & $\begin{array}{l}\mathcal{N} \\
{\left[10^{45}\right]}\end{array}$ & & & & & \\
\hline IRS7A & $294^{b} / 682^{b}$ & $35.7^{b}$ & $83^{b}$ & $132^{b}$ & $197^{b}$ & $10.1^{b}$ & $79^{b}$ & $9.1^{b}$ & yes $/$ yes $^{b} /$ no $^{c}$ & yes $^{b} /$ yes & 0/I \\
\hline SMM 1C & - & - & - & - & - & - & - & - & no/yes ${ }^{b} /$ yes & yes $^{b} /$ yes & $0 / \mathrm{I}$ \\
\hline IRS7B & $273 / 710$ & 12.5 & 89 & 17 & 174 & 2.3 & 89 & 4.6 & yes/yes/yes & yes/yes & $0 / \mathrm{I}$ \\
\hline $\mathrm{R} \mathrm{CrA}$ & 287 / 992 & 5.7 & 99 & 7.1 & 235 & 1.3 & 889 & 53 & yes/yes/faint & yes/yes & II/III \\
\hline IRS5A & $293 / 1417$ & 3.2 & 80 & 17 & 185 & 1.2 & 209 & 1.7 & yes/yes/yes & yes/... & $0 / I$ \\
\hline IRS5N & $283 / \ldots$ & 0.7 & $\ldots$ & $\ldots$ & 195 & 0.5 & 63 & 0.7 & no/yes/yes & no/... & 0 \\
\hline Res SW-1 & $285 / 653$ & 5.0 & 76 & 9. & 181 & 2. & $\cdots$ & $\cdots$ & $\cdots$ & $\ldots$ & $\ldots$ \\
\hline Res E-1 & $287 / 1015$ & 1.7 & 68 & 7.2 & 201 & 8.6 & $\ldots$ & $\ldots$ & $\ldots$ & $\ldots$ & $\ldots$ \\
\hline Res N-1 & $281 / 898$ & 3.3 & 66 & 19 & 174 & 2.3 & $\ldots$ & $\ldots$ & $\ldots$ & $\ldots$ & $\ldots$ \\
\hline Res W-1 & $253 / 751$ & 13.0 & 72 & 26 & 235 & 1.3 & $\ldots$ & $\ldots$ & $\ldots$ & $\ldots$ & $\ldots$ \\
\hline DIGIT $^{d}$ & $355 / 814$ & 7.0 & 83 & 24 & 194 & 7.7 & 167 & 6 & $\ldots$ & $\ldots$ & $\ldots$ \\
\hline
\end{tabular}

Notes. ${ }^{(a)}$ The temperatures are for the warm and hot components, respectively. The total number of molecules is the sum of the warm and hot components. ${ }^{(b)}$ Combined value for IRS7A and SMM 1C. ${ }^{(c)}$ Detected in ALMA $0.8 \mathrm{~mm}$ continuum (Lindberg et al. 2013). ${ }^{(d)}$ Average of the DIGIT sample, from Green et al. (2013), not including the CrA and Serpens sources. The OH rotational diagrams have been recalculated excluding the same lines that are excluded in this paper.

In the last row of Fig. 18, the [O I] $63.2 \mu$ m line flux is compared to two CO lines. In the comparison of [O I] and $\mathrm{CO}$ it is more difficult to accommodate our ambition of using similar wavelengths and upper-level energies; we could have used the [O I] $145 \mu \mathrm{m}$ line, but this was not desirable for $\mathrm{S} / \mathrm{N}$ reasons. We find that the [OI] flux is enhanced in the CrA sources also with respect to the $\mathrm{CO}$ flux.

The higher $[\mathrm{OI}] / \mathrm{OH},[\mathrm{OI}] / \mathrm{H}_{2} \mathrm{O}$, and $\mathrm{OH} / \mathrm{H}_{2} \mathrm{O}$ line ratios could also indicate a later stage of evolution (cf. Class II sources in Podio et al. 2012), but the low bolometric temperatures and $L_{\text {bol }} / L_{\text {submm }}$ ratios of the sources indicate that they are Class 0/I sources, and the (in many cases) higher line ratios found in the extended emission compared to the point sources indicate that the heating is external in its origin.

\section{Conclusions}

We study Herschel/PACS line and continuum maps of the lowmass star-forming region $\mathrm{R}$ CrA subject to strong irradiation from the nearby Herbig Be star R CrA. In addition, we deconvolve the maps to study the point-source and extended contributions to the emission. Our main results are the following:

1. FIR continuum emission is found not only at the (sub)mm and MIR continuum point sources, but also (somewhat fainter) in two ridges north and south of the IRS7 protostars. These correlate in position with $\mathrm{H}_{2} \mathrm{CO}$ and $\mathrm{CH}_{3} \mathrm{OH}$ $\mathrm{mm}$ emission, and the continuum emission peaks give temperatures $(40-50 \mathrm{~K})$ similar to the rotational temperature of the $\mathrm{H}_{2} \mathrm{CO}$ emission (Lindberg \& Jørgensen 2012), both suggesting that the extended FIR continuum emission traces the dust associated with the externally irradiated material.

2. The rotational temperatures of the warm $\mathrm{CO}$ component $(286 \pm 3 \mathrm{~K})$, the hot $\mathrm{CO}$ component $(950 \pm 148 \mathrm{~K}), \mathrm{OH}$ $(88 \pm 4 \mathrm{~K})$, and $\mathrm{H}_{2} \mathrm{O}(197 \pm 9 \mathrm{~K})$ measured towards the continuum point sources are consistent with or lower than those found in larger samples of similar sources, suggesting that the excitation conditions of the dense gas close to the protostars are not affected by the external irradiation. A
${ }^{13} \mathrm{CO}$ rotational diagram suggests that the mid- $J{ }^{12} \mathrm{CO}$ lines are marginally optically thick $(\tau \sim 0.6)$.

3. $\mathrm{CO}, \mathrm{OH}$, and $\mathrm{H}_{2} \mathrm{O}$ emission not associated with any of the previously known continuum point sources is detected, and shows excitation conditions similar to the gas near the protostars $(277 \pm 7 \mathrm{~K}$ for the warm CO component, $829 \pm 69 \mathrm{~K}$ for the hot $\mathrm{CO}$ component, $71 \pm 2 \mathrm{~K}$ for $\mathrm{OH}$, and $198 \pm 12$ for $\mathrm{H}_{2} \mathrm{O}$ ). The warm gas thus exists on much larger scales than can be explained by heating from the low-mass YSOs. One possible explanation is that this emission traces radiatively excited low-density gas, but detections of high density tracers such as $\mathrm{H}_{2} \mathrm{CO}$ and $\mathrm{CH}_{3} \mathrm{OH}$ challenge this hypothesis. The extent of the FIR molecular emission is larger than previously seen in any low-mass protostellar sources.

4. When comparing the IRS7 and IRS5 fields - the former with a smaller angular separation from the irradiating Herbig Be star R CrA than the latter - we find that the two fields have similar average rotational temperatures of the warm $\mathrm{CO}$ component ( $285 \mathrm{~K}$ and $288 \mathrm{~K}$, respectively), $\mathrm{OH}(90 \mathrm{~K}$ and $80 \mathrm{~K}$, respectively), and $\mathrm{H}_{2} \mathrm{O}(200 \mathrm{~K}$ and $190 \mathrm{~K}$, respectively). However, more extended emission (both line and continuum) is seen in the IRS7 field than in the IRS5 field. The higher level of irradiation from R CrA in IRS7 than in IRS5 does not significantly affect the rotational temperatures, but the possible link between extended emission and external irradiation needs to be investigated further.

5. The $\mathrm{OH} / \mathrm{H}_{2} \mathrm{O},[\mathrm{OI}] / \mathrm{H}_{2} \mathrm{O},[\mathrm{OI}] / \mathrm{OH}$, and $[\mathrm{OI}] / \mathrm{CO}$ line ratios are enhanced in the CrA point sources and extended gas compared to other embedded objects, which is similar to what has previously been seen in PDRs (Hollenbach \& Tielens 1997). Typically, these line ratios are enhanced by a factor of 1.5-4.0 in the CrA sources.

To further study the origin of the excitation conditions in protostellar cores and their surroundings, we propose similar investigations of any extended emission in PACS observations of embedded objects, including other sources in regions of potential strong external irradiation. This could include for instance the 
J. E. Lindberg et al.: Warm gas towards young stellar objects in Corona Australis

$\mathrm{OH} 84.6 \mu \mathrm{m} / \mathrm{H}_{2} \mathrm{O} 75.4 \mu \mathrm{m}$

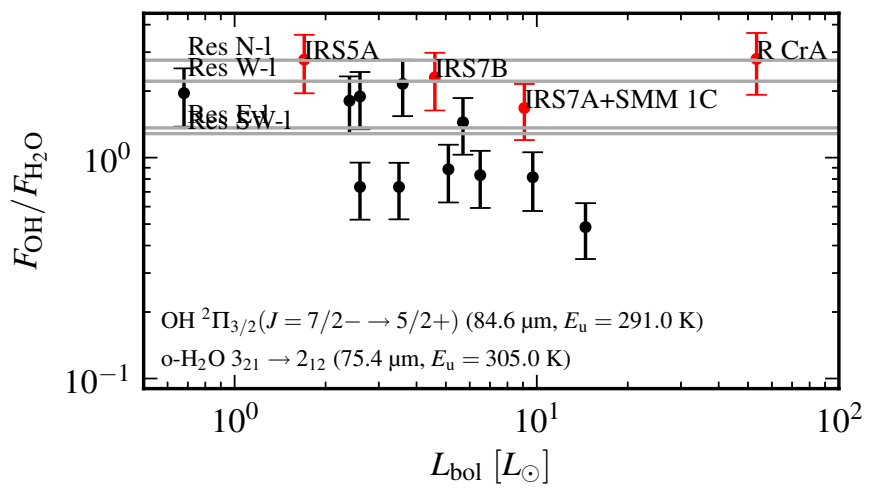

OH $65.3 \mu \mathrm{m} / \mathrm{H}_{2} \mathrm{O} 78.7 \mu \mathrm{m}$

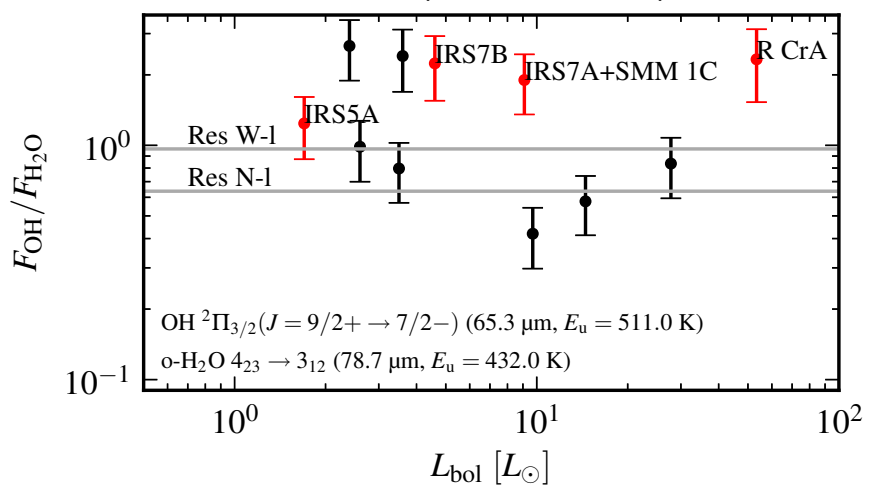

[O I] $63.2 \mu \mathrm{m} / \mathrm{H}_{2} \mathrm{O} 75.4 \mu \mathrm{m}$

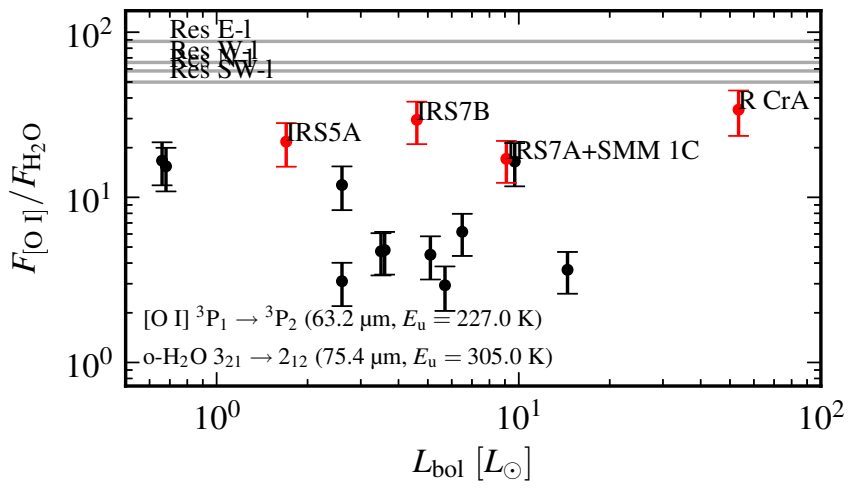

[O I] $63.2 \mu \mathrm{m} / \mathrm{CO} 186.0 \mu \mathrm{m}$

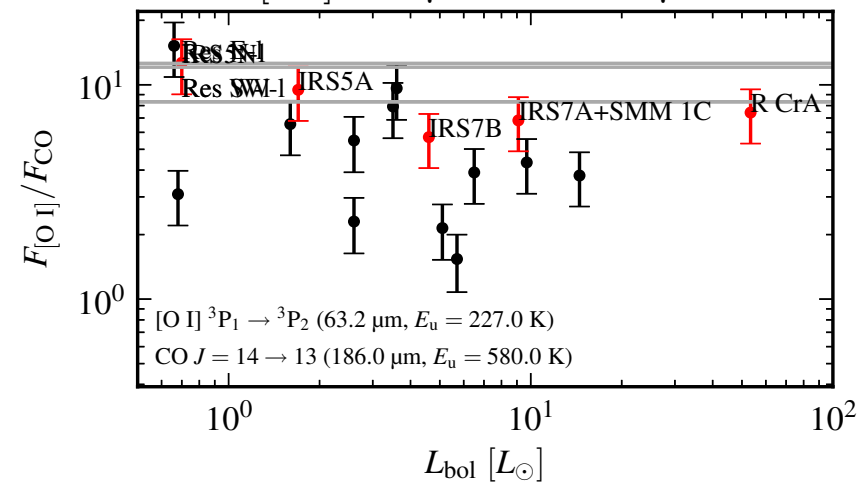

$\mathrm{OH} 84.6 \mu \mathrm{m} / \mathrm{H}_{2} \mathrm{O} 90.0 \mu \mathrm{m}$

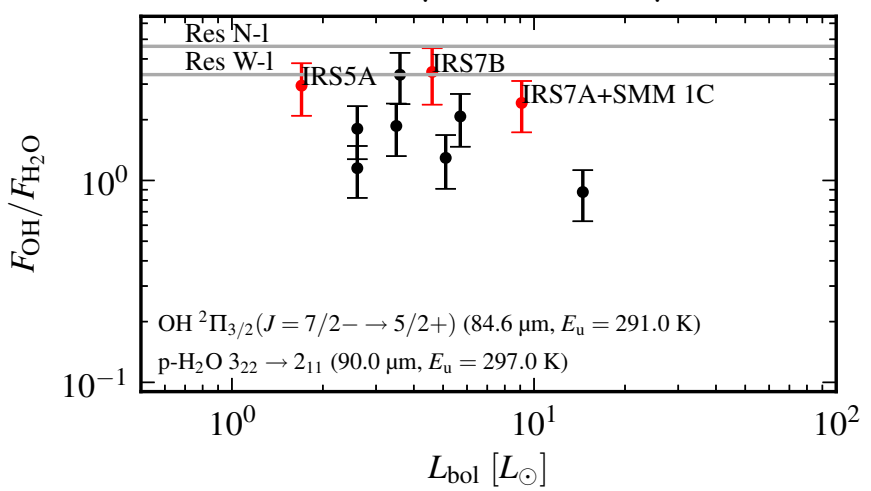

OH $163.4 \mu \mathrm{m} / \mathrm{H}_{2} \mathrm{O} 180.5 \mu \mathrm{m}$

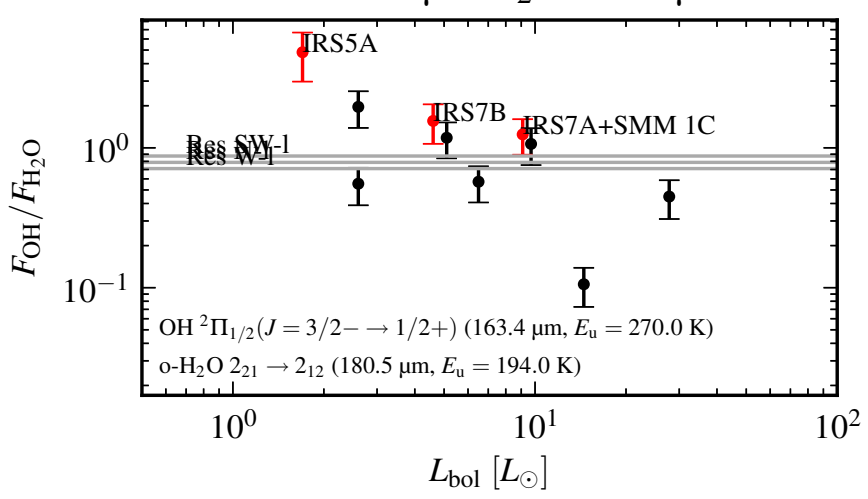

[O I] $63.2 \mu \mathrm{m} / \mathrm{OH} 84.6 \mu \mathrm{m}$

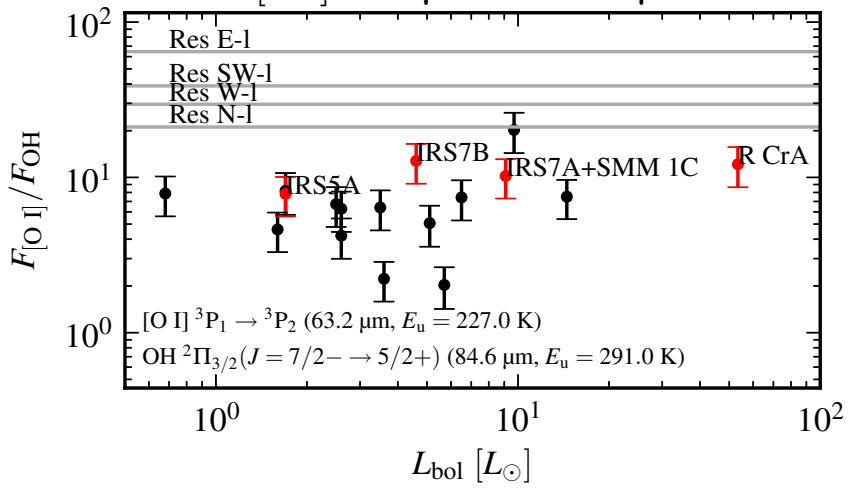

[O I] $63.2 \mu \mathrm{m} / \mathrm{CO} 118.6 \mu \mathrm{m}$

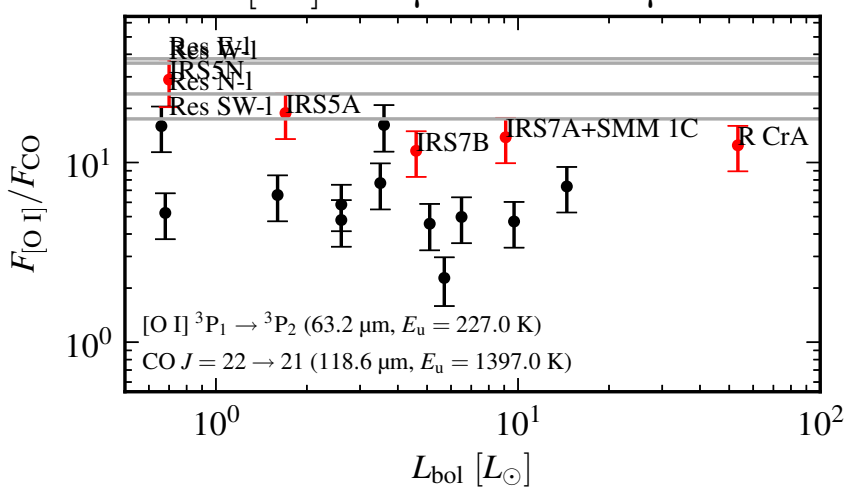

Fig. 18. Ratios of [O I], OH, $\mathrm{H}_{2} \mathrm{O}$, and $\mathrm{CO}$ lines plotted versus the bolometric luminosities of the sources. See details in the figures. The CrA point sources are marked with red; the black data points are other DIGIT embedded sources (Green et al. 2013). The CrA extended emission regions are marked with grey lines. Only data points where both lines are significantly detected are included. 
Orion sources discussed by Manoj et al. (2013), and the isolated sources in the DIGIT sample (Green et al. 2013).

Acknowledgements. This research was supported by a grant from the Instrument Center for Danish Astrophysics (IDA) and a Lundbeck Foundation Group Leader Fellowship to J.K.J. Research at Centre for Star and Planet Formation is funded by the Danish National Research Foundation and the University of Copenhagen's programme of excellence. Support for this work, part of the Herschel Open Time Key Project Program, was provided by NASA through an award issued by the Jet Propulsion Laboratory, California Institute of Technology. The William Herschel Telescope is operated on the island of La Palma by the Isaac Newton Group in the Spanish Observatorio del Roque de los Muchachos of the Instituto de Astrofísica de Canarias. We thank Nienke van der Marel for helping us obtain the optical spectrum of R CrA. The authors would also like to thank the anonymous referee, Lars Kristensen, and Tim van Kempen for their useful suggestions and comments which improved the quality of the paper.

\section{References}

André, P., Ward-Thompson, D., \& Barsony, M. 1993, ApJ, 406, 122 Bibo, E. A., Thé, P. S., \& Dawanas, D. N. 1992, A\&A, 260, 293 Brown, A. 1987, ApJ, 322, L31

Brown, J. M., Pontoppidan, K. M., van Dishoeck, E. F., et al. 2013, ApJ, 770, 94 Dionatos, O., Jørgensen, J. K., Green, J. D., et al. 2013, A\&A, 558, A88 Forbrich, J., \& Preibisch, T. 2007, A\&A, 475, 959

Giannini, T., Lorenzetti, D., Tommasi, E., et al. 1999, A\&A, 346, 617 Goicoechea, J. R., Cernicharo, J., Karska, A., et al. 2012, A\&A, 548, A77 Goldsmith, P. F., \& Langer, W. D. 1999, ApJ, 517, 209

Gray, R. O., Corbally, C. J., Garrison, R. F., et al. 2006, AJ, 132, 161 Green, J. D., Evans, II, N. J., Jørgensen, J. K., et al. 2013, ApJ, 770, 123 Greenstein, J. L., \& Aller, L. H. 1947, PASP, 59, 139

Groppi, C. E., Hunter, T. R., Blundell, R., \& Sandell, G. 2007, ApJ, 670, 489

Harju, J., Haikala, L. K., Mattila, K., et al. 1993, A\&A, 278, 569

Herbst, W., \& Shevchenko, V. S. 1999, AJ, 118, 1043

Herczeg, G. J., Karska, A., Bruderer, S., et al. 2012, A\&A, 540, A84

Hillenbrand, L. A., Strom, S. E., Vrba, F. J., \& Keene, J. 1992, ApJ, 397, 613

Högbom, J. A. 1974, A\&AS, 15, 417

Hollenbach, D. J., \& Tielens, A. G. G. M. 1997, ARA\&A, 35, 179

Jensen, E. L. N., Mathieu, R. D., \& Fuller, G. A. 1996, ApJ, 458, 312

Jørgensen, J. K., van Dishoeck, E. F., Visser, R., et al. 2009, A\&A, 507, 861 Joy, A. H. 1945, ApJ, 102, 168

Karska, A., Herczeg, G. J., van Dishoeck, E. F., et al. 2013, A\&A, 552, A141

Kaufman, M. J., Wolfire, M. G., Hollenbach, D. J., \& Luhman, M. L. 1999, ApJ, 527,795
Koen, C., Kilkenny, D., van Wyk, F., \& Marang, F. 2010, MNRAS, 403, 1949 Kristensen, L. E., van Dishoeck, E. F., Bergin, E. A., et al. 2012, A\&A, 542, A8 Lee, J., Lee, J.-E., Lee, S., et al. 2013, ApJS, 209, 4

Lindberg, J. E., \& Jørgensen, J. K. 2012, A\&A, 548, A24

Lindberg, J. E., Jørgensen, J. K., Brinch, C., et al. 2013, A\&A, submitted

Loren, R. B. 1979, ApJ, 227, 832

Lorenzetti, D., Tommasi, E., Giannini, T., et al. 1999, A\&A, 346, 604

Manoj, P., Bhatt, H. C., Maheswar, G., \& Muneer, S. 2006, ApJ, 653, 657

Manoj, P., Watson, D. M., Neufeld, D. A., et al. 2013, ApJ, 763, 83

Myers, P. C., Adams, F. C., Chen, H., \& Schaff, E. 1998, ApJ, 492, 703

Neufeld, D. A. 2012, ApJ, 749, 125

Neuhäuser, R., \& Forbrich, J. 2008, in Handbook of Star Forming Regions, Vol. II: The Southern Sky, ed. B. Reipurth (San Francisco, CA: ASP Monographs), 735

Nutter, D. J., Ward-Thompson, D., \& André, P. 2005, MNRAS, 357, 975

Peterson, D. E., Caratti o Garatti, A., Bourke, T. L., et al. 2011, ApJS, 194, 43

Pickles, A. J. 1998, PASP, 110, 863

Pilbratt, G. L., Riedinger, J. R., Passvogel, T., et al. 2010, A\&A, 518, L1

Podio, L., Kamp, I., Flower, D., et al. 2012, A\&A, 545, A44

Poglitsch, A., Waelkens, C., Geis, N., et al. 2010, A\&A, 518, L2

Robitaille, T. P., Whitney, B. A., Indebetouw, R., Wood, K., \& Denzmore, P. 2006, ApJS, 167, 256

Robitaille, T. P., Whitney, B. A., Indebetouw, R., \& Wood, K. 2007, ApJS, 169, 328

Schöier, F. L., van der Tak, F. F. S., van Dishoeck, E. F., \& Black, J. H. 2005 , A\&A, 432, 369

Schöier, F. L., Jørgensen, J. K., Pontoppidan, K. M., \& Lundgren, A. A. 2006, A\&A, 454, L67

Sicilia-Aguilar, A., Henning, T., Linz, H., et al. 2013, A\&A, 551, A34

Skrutskie, M. F., Cutri, R. M., Stiening, R., et al. 2006, AJ, 131, 1163

Sturm, B., Bouwman, J., Henning, T., et al. 2010, A\&A, 518, L129

Taylor, K. N. R., \& Storey, J. W. V. 1984, MNRAS, 209, 5

van der Tak, F. F. S., Black, J. H., Schöier, F. L., Jansen, D. J., \& van Dishoeck, E. F. 2007 , A\&A, 468, 627

van Kempen, T. A., van Dishoeck, E. F., Güsten, R., et al. 2009a, A\&A, 507, 1425

van Kempen, T. A., van Dishoeck, E. F., Hogerheijde, M. R., \& Güsten, R. 2009b, A\&A, 508, 259

van Kempen, T. A., Kristensen, L. E., Herczeg, G. J., et al. 2010, A\&A, 518, L121

Vieira, S. L. A., Corradi, W. J. B., Alencar, S. H. P., et al. 2003, AJ, 126, 2971

Walsh, C., Millar, T. J., \& Nomura, H. 2013, ApJ, 766, L23

Wampfler, S. F., Bruderer, S., Karska, A., et al. 2013, A\&A, 552, A56

Weingartner, J. C., \& Draine, B. T. 2001, ApJ, 548, 296

Wilson, T. L., \& Rood, R. 1994, ARA\&A, 32, 191 


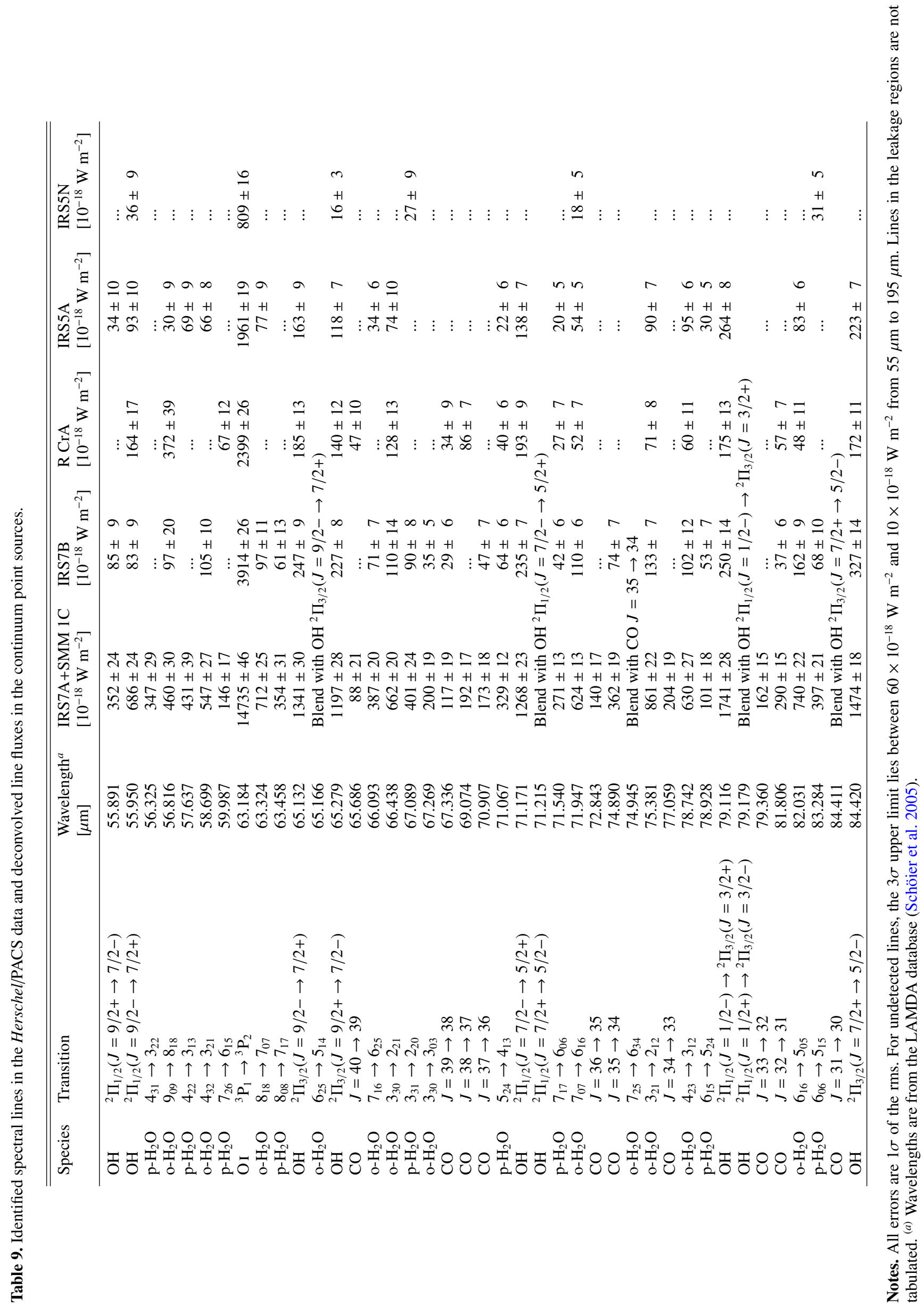




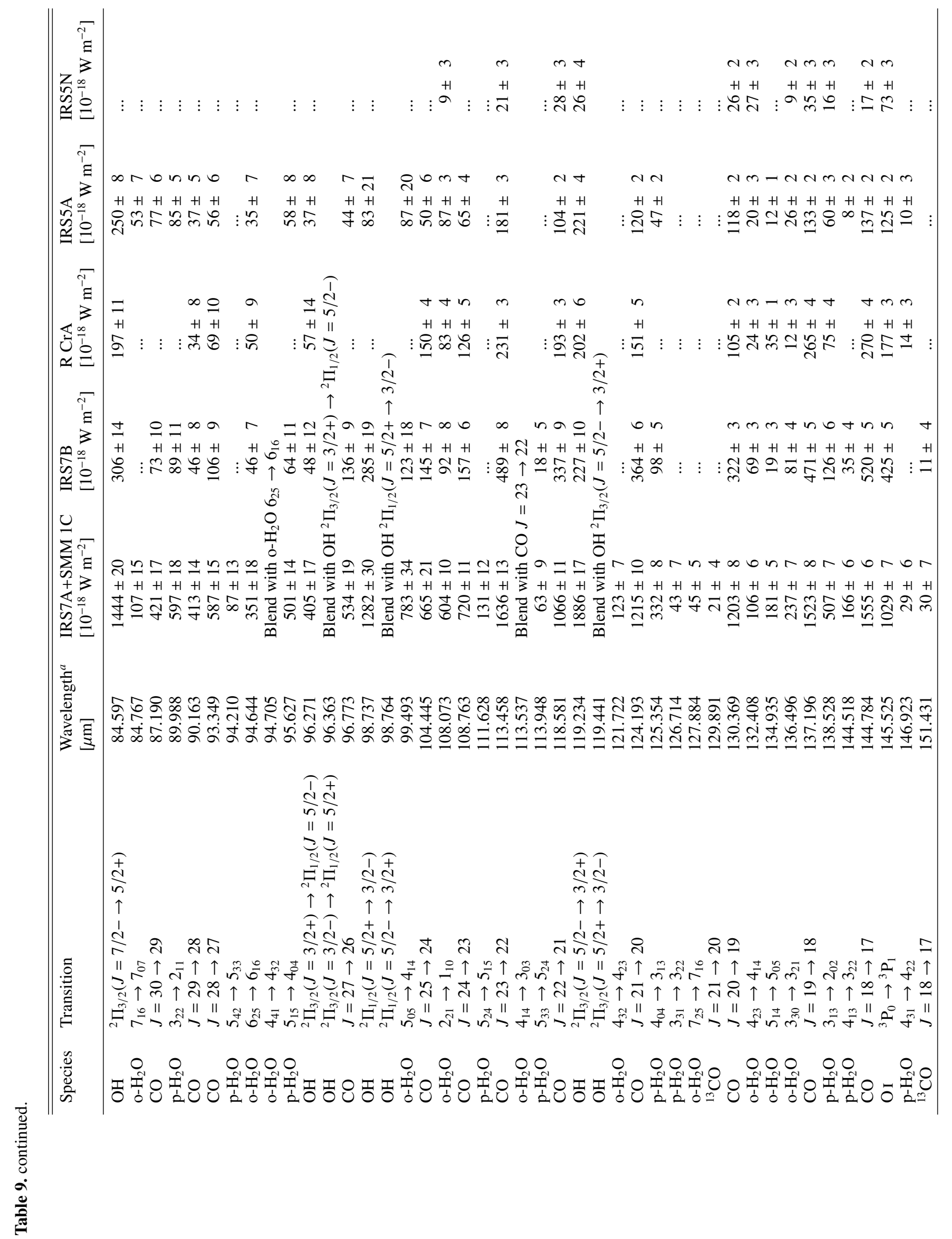




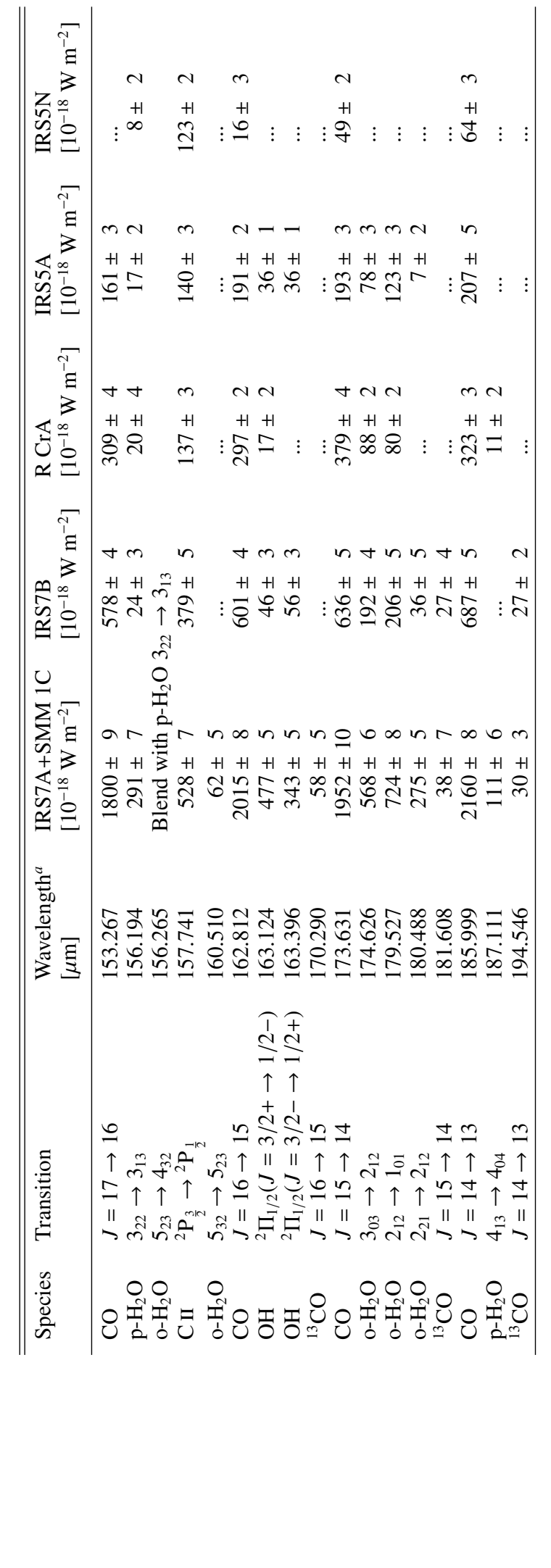




\section{Appendix A: Continuum maps}

The PACS continuum maps and POMAC residuals are found in Figs. A.1 and A.2.
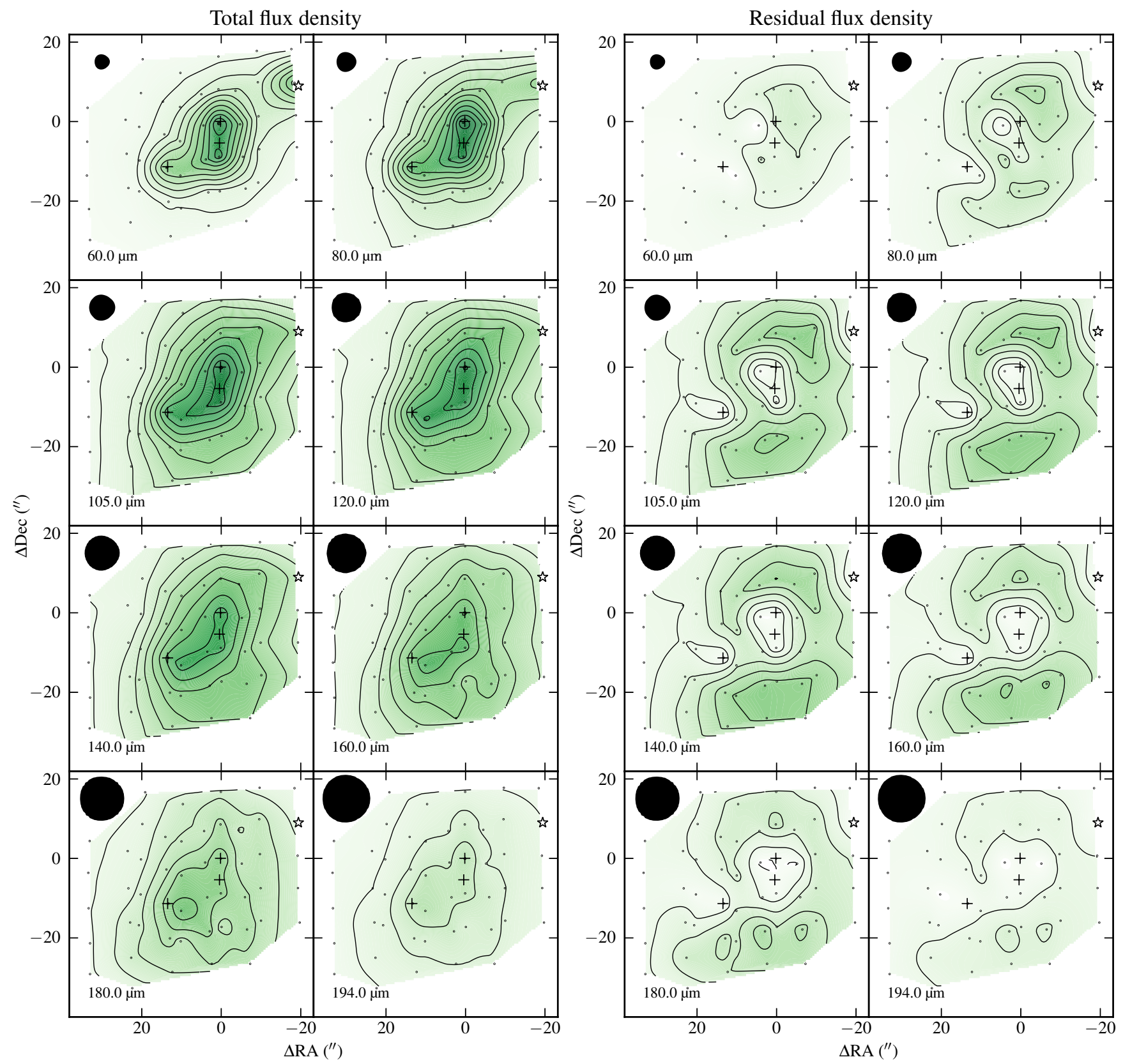

Fig. A.1. Continuum maps of the IRS7 region. Left: total flux density map. Right: residual map. Contour levels are at $10 \mathrm{Jy}$ for all maps. The Herschel PSF for each observation is shown in the top left corner. 
J. E. Lindberg et al.: Warm gas towards young stellar objects in Corona Australis
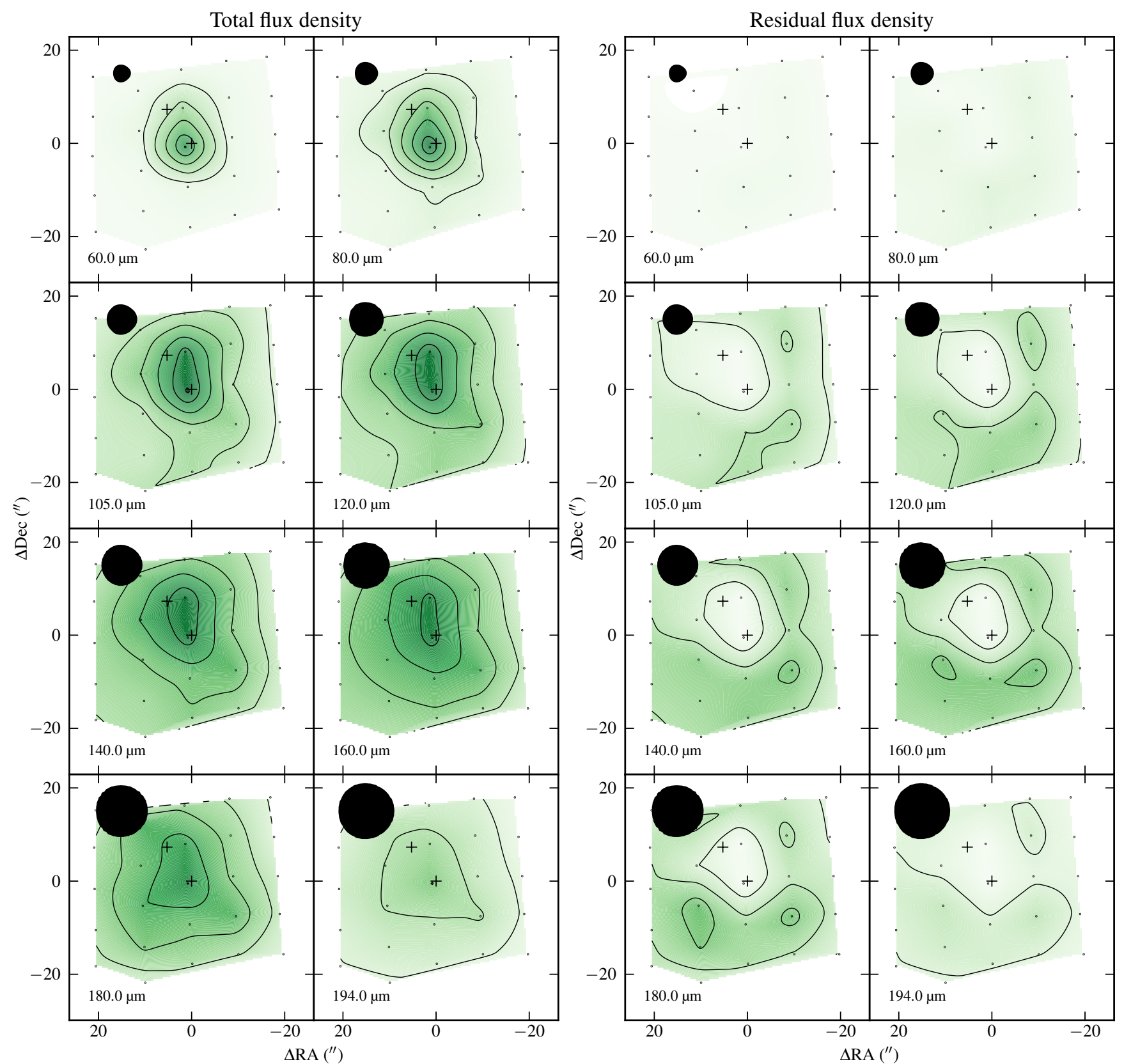

Fig. A.2. Continuum maps of the IRS5 region. Left: total flux density map. Right: residual map. Contour levels are at 3 Jy for all maps. The Herschel PSF for each observation is shown in the top left corner. 


\section{Appendix B: Line maps}

The line flux maps and POMAC residual maps of the CO lines are found in Figs. B.1 and B.2, the OH maps in Figs. B.3 and B.4, the $\mathrm{H}_{2} \mathrm{O}$ maps in Figs. B.5-B.8, and the [O I] and [C II] maps in Figs. B.9 and B.10.
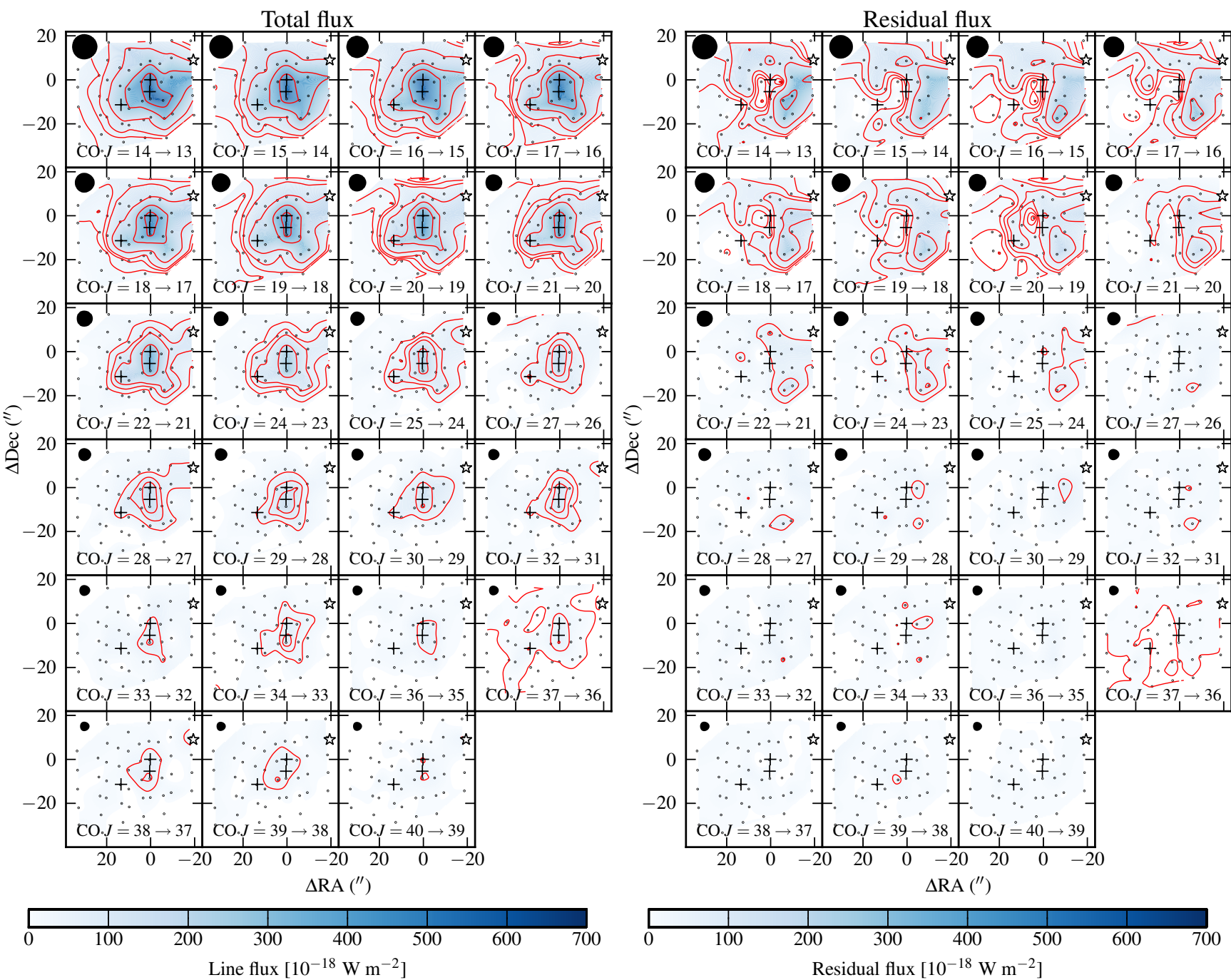

Fig. B.1. CO line maps of the IRS7 region. Left: total flux map. Right: residual map. The red contour levels are at $5 \sigma, 10 \sigma, 15 \sigma, 30 \sigma, 60 \sigma$, and $90 \sigma$ (dashed contours for negative fluxes). The blue colour maps have the same scale for all maps. The Herschel PSF for each observation is shown in the top left corner. 
J. E. Lindberg et al.: Warm gas towards young stellar objects in Corona Australis
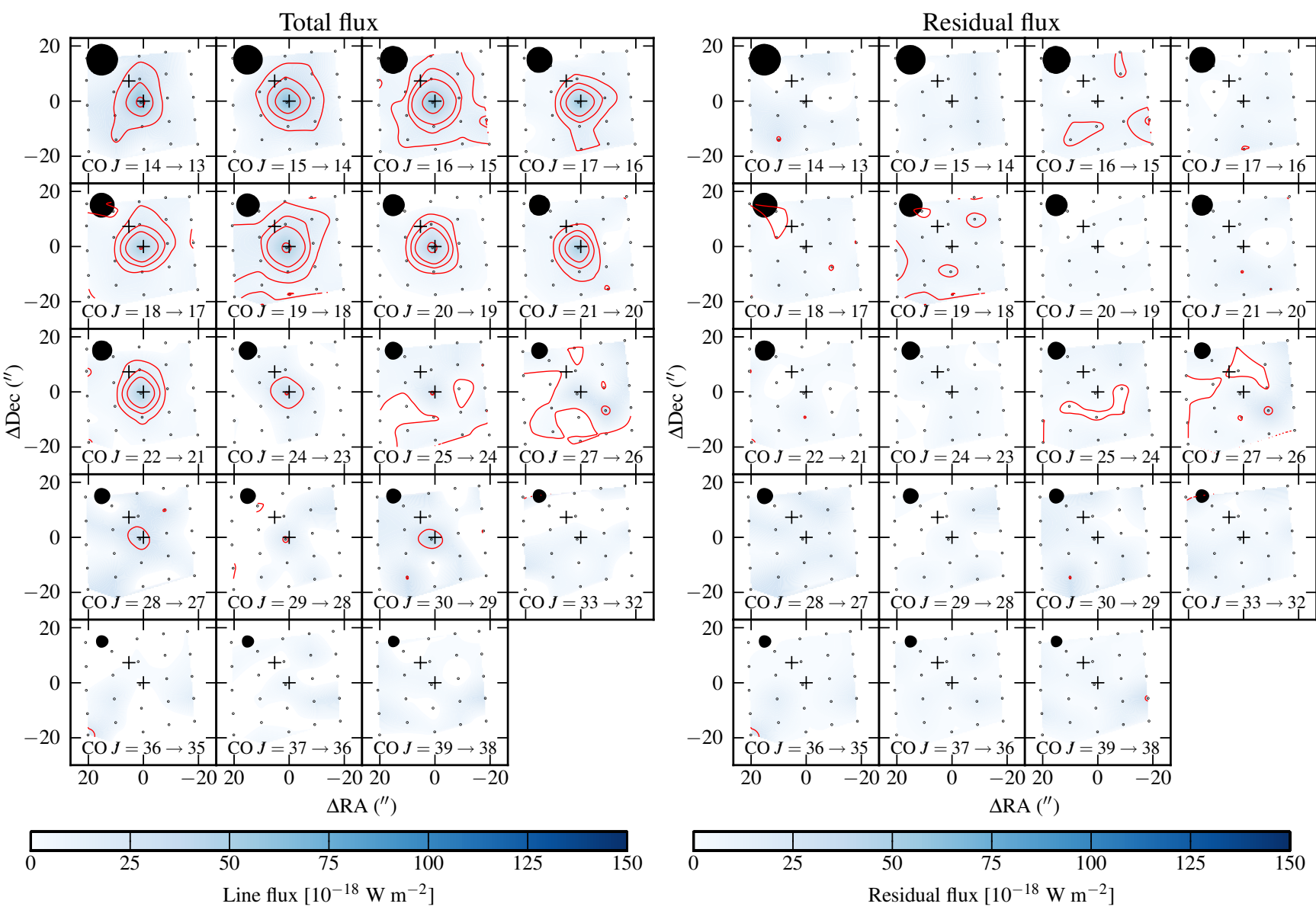

Fig. B.2. CO line maps of the IRS5 region. Left: total flux map. Right: residual map. Contour levels as in Fig. B.1. The blue colour maps have the same scale for all maps. The Herschel PSF for each observation is shown in the top left corner.
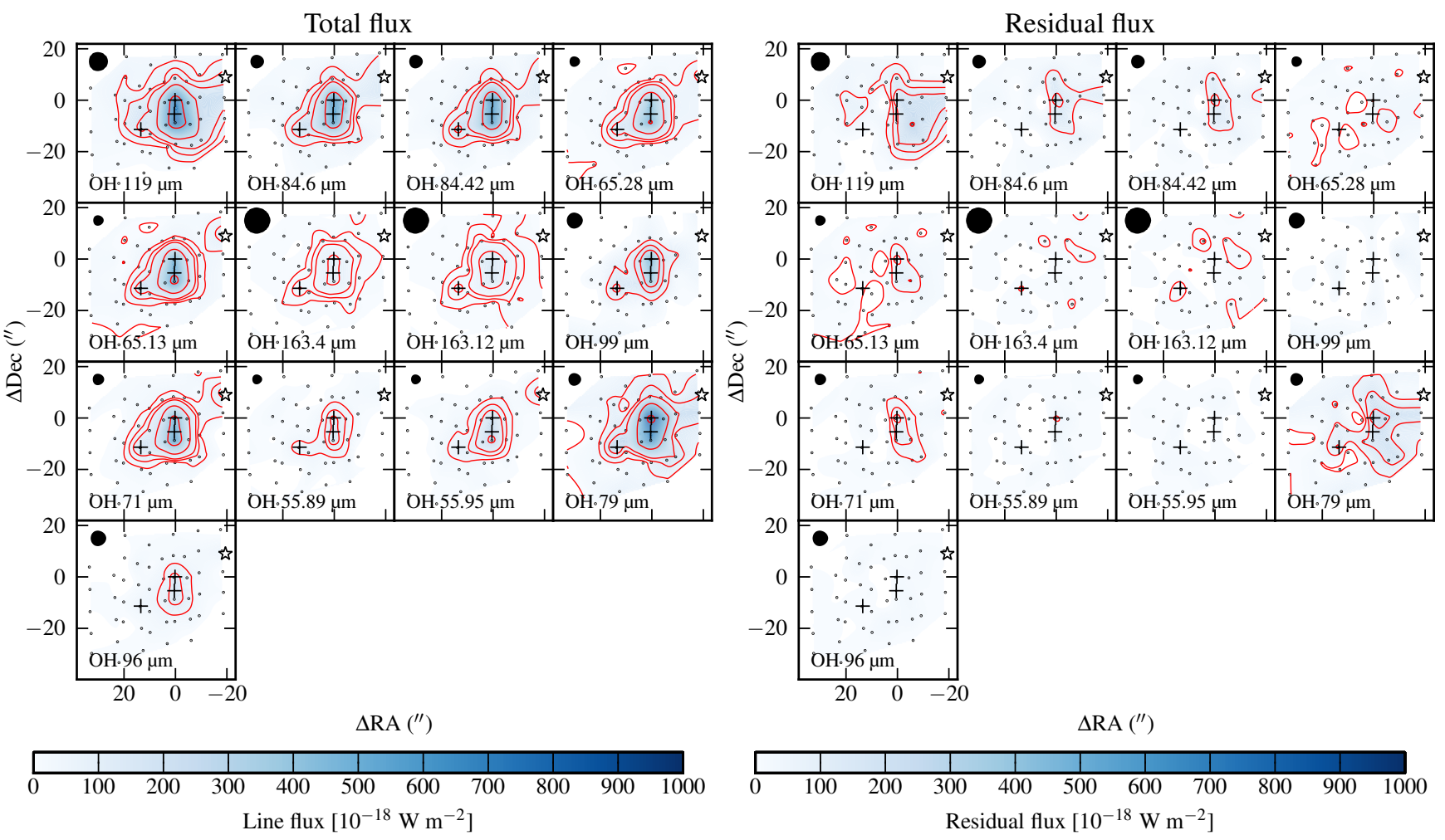

Fig. B.3. OH line maps of the IRS7 region. Left: total flux map. Right: residual map. Contour levels as in Fig. B.1. The blue colour maps have the same scale for all maps. The Herschel PSF for each observation is shown in the top left corner. 

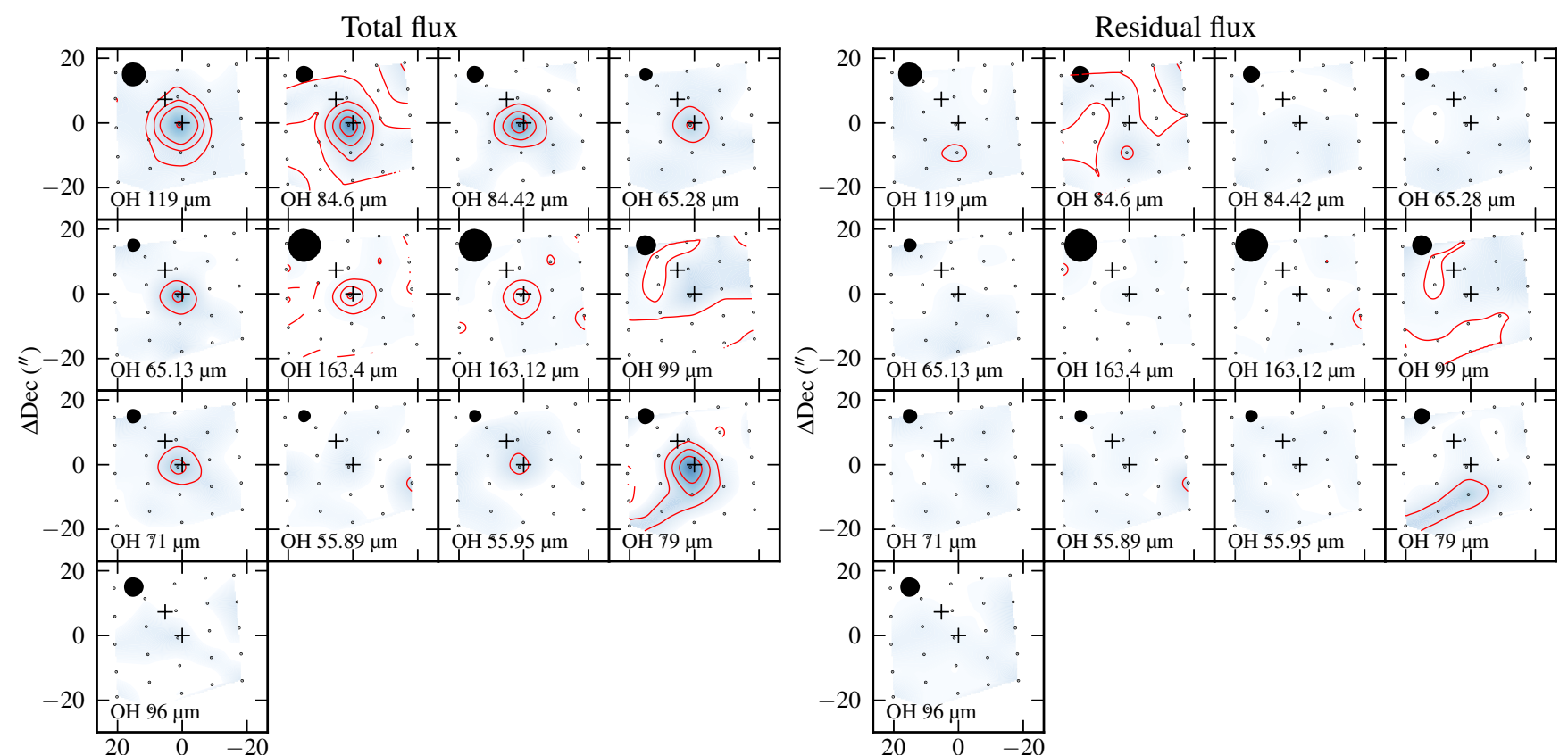

$\Delta \mathrm{RA}\left({ }^{\prime \prime}\right)$
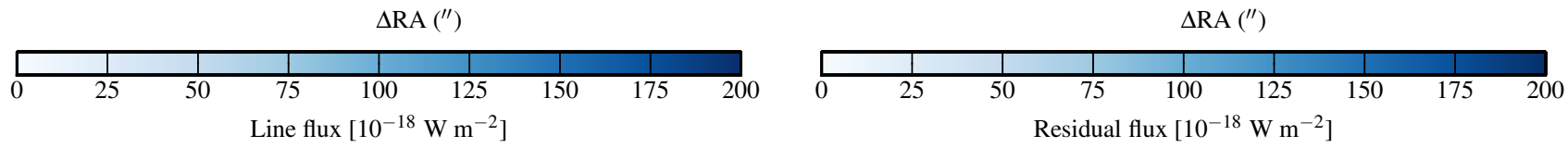

Fig. B.4. OH line maps of the IRS5 region. Left: total flux map. Right: residual map. Contour levels as in Fig. B.1. The blue colour maps have the same scale for all maps. The Herschel PSF for each observation is shown in the top left corner.
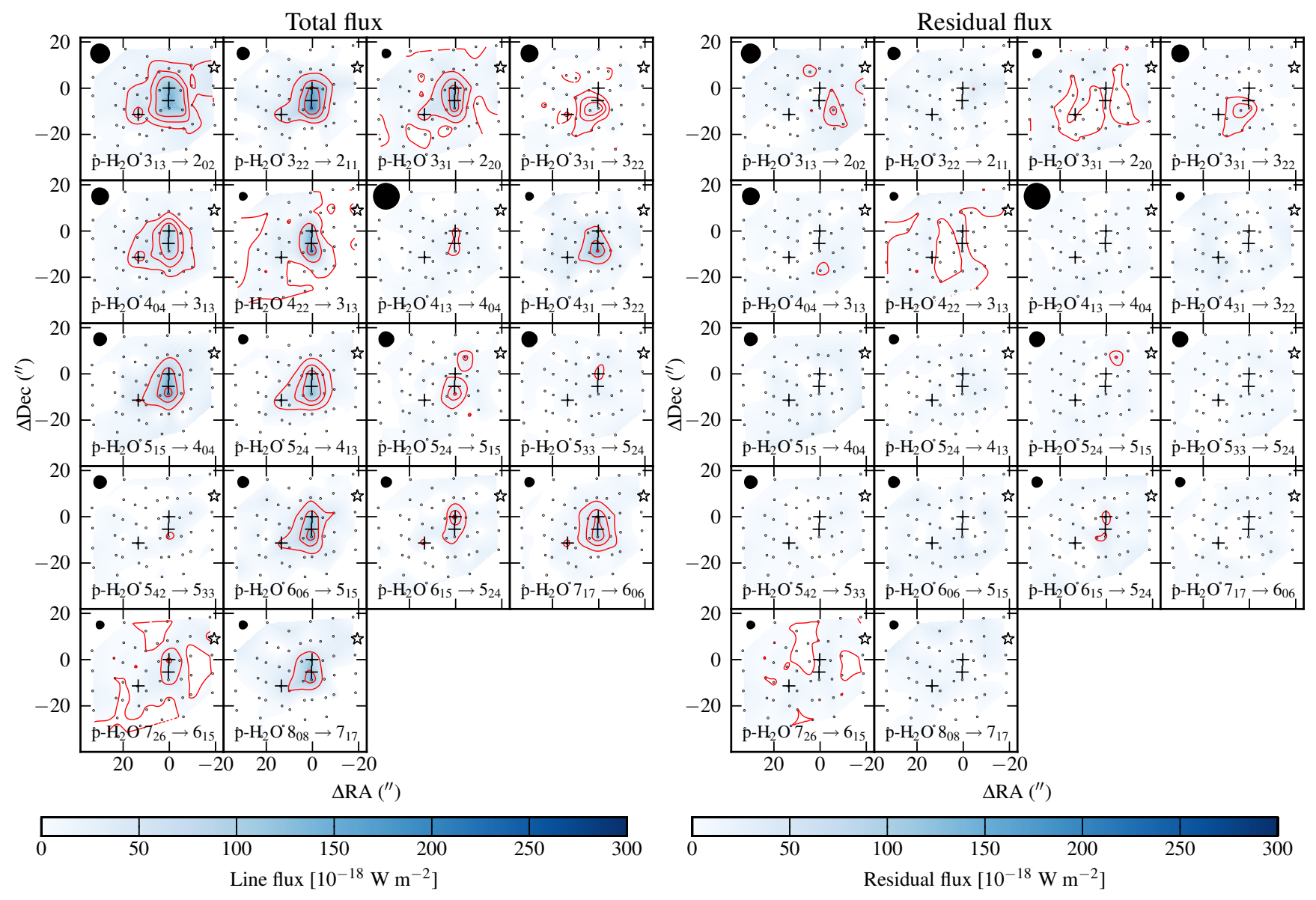

Fig. B.5. p- $\mathrm{H}_{2} \mathrm{O}$ line maps of the IRS7 region. Left: total flux map. Right: residual map. Contour levels as in Fig. B.1.The blue colour maps have the same scale for all maps. The Herschel PSF for each observation is shown in the top left corner. 
J. E. Lindberg et al.: Warm gas towards young stellar objects in Corona Australis
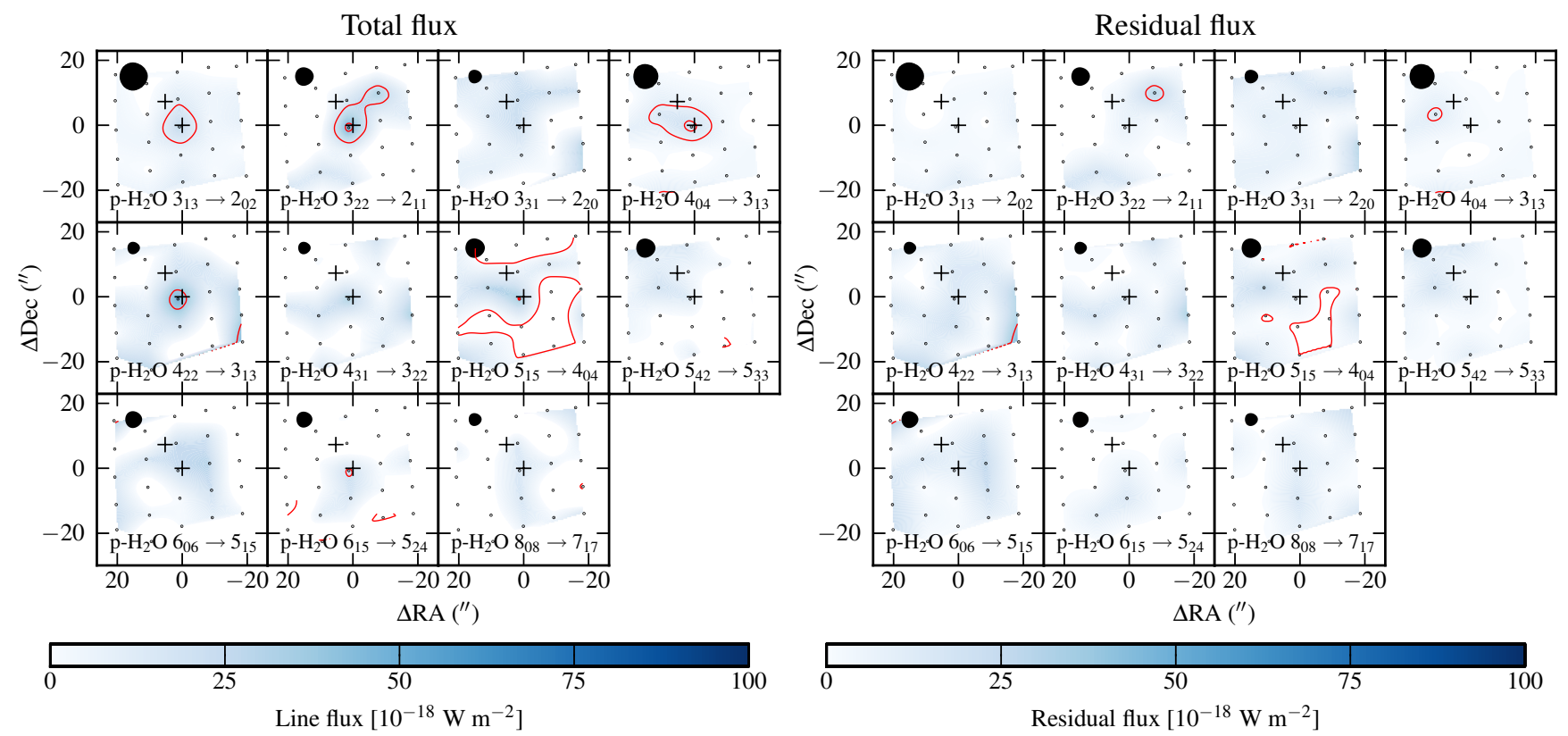

Fig. B.6. p- $\mathrm{H}_{2} \mathrm{O}$ line maps of the IRS5 region. Left: total flux map. Right: residual map. Contour levels as in Fig. B.1. The blue colour maps have the same scale for all maps. The Herschel PSF for each observation is shown in the top left corner.
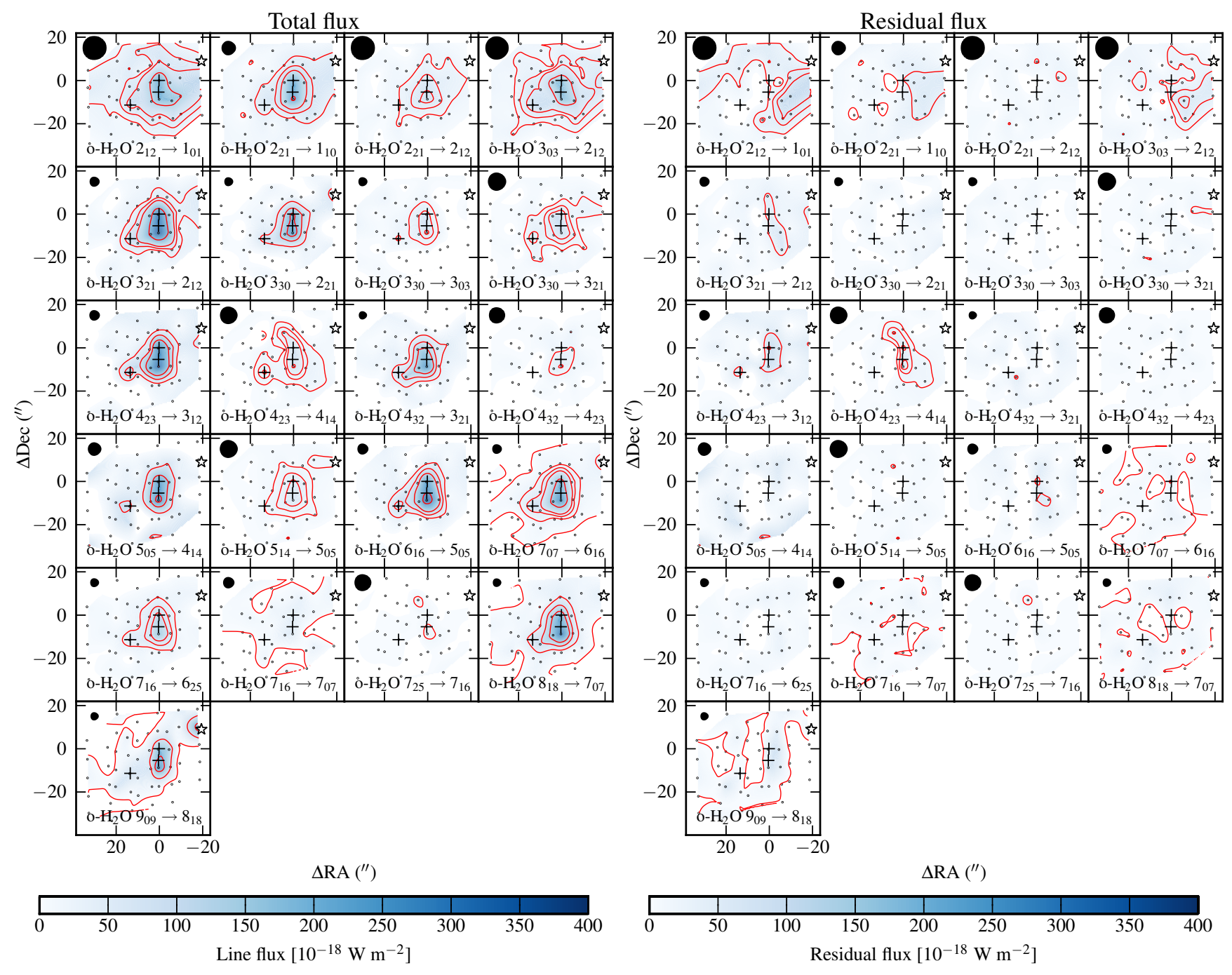

Fig. B.7. o- $\mathrm{H}_{2} \mathrm{O}$ line maps of the IRS7 region. Left: total flux map. Right: residual map. Contour levels as in Fig. B.1. The blue colour maps have the same scale for all maps. The Herschel PSF for each observation is shown in the top left corner. 

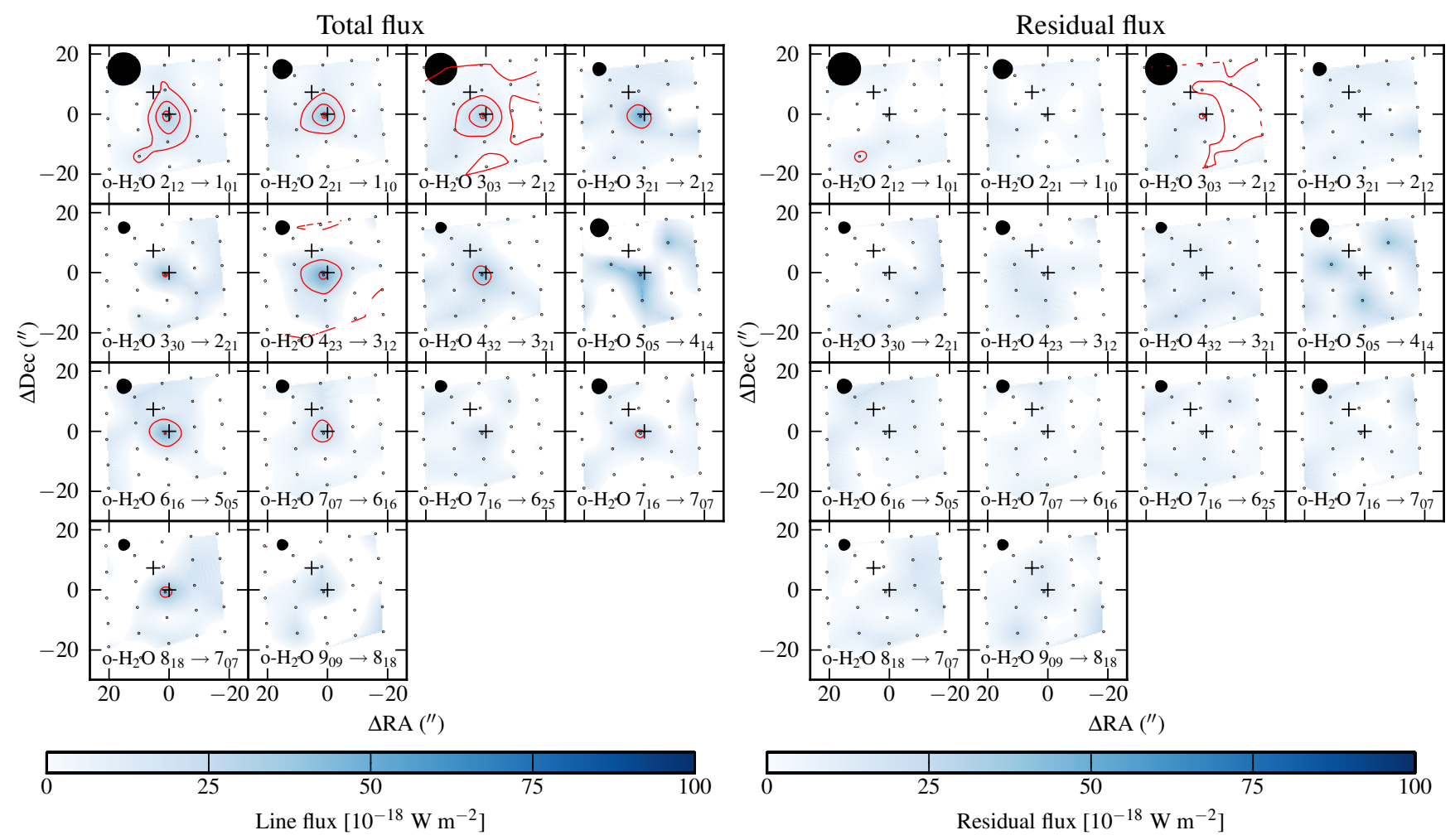

Fig. B.8. o- $\mathrm{H}_{2} \mathrm{O}$ line maps of the IRS5 region. Left: total flux map. Right: residual map. Contour levels as in Fig. B.1. The blue colour maps have the same scale for all maps. The Herschel PSF for each observation is shown in the top left corner.

Total flux
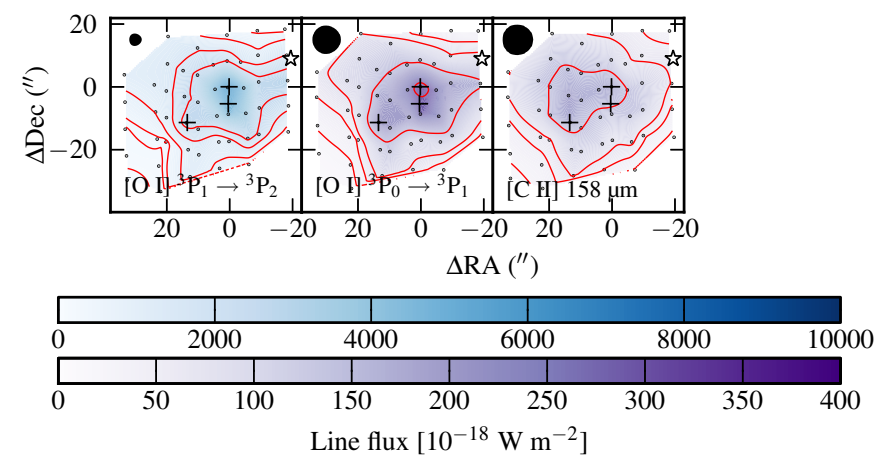

Residual flux
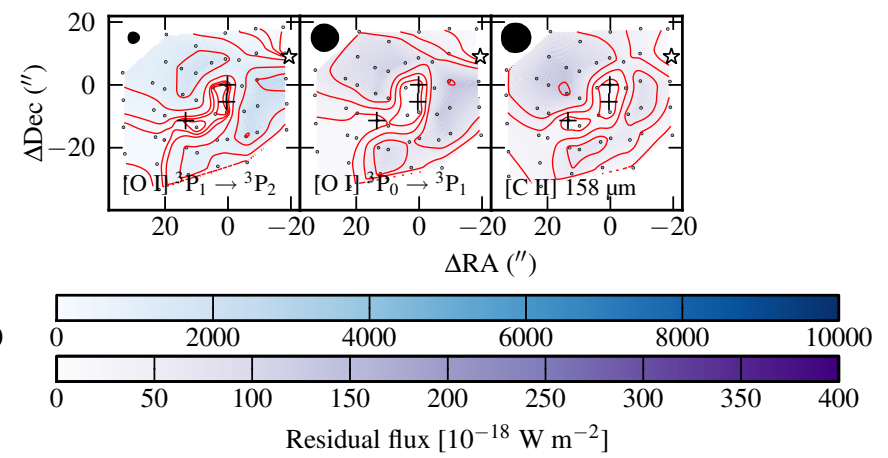

Fig. B.9. Atomic line maps of the IRS7 region. Left: total flux map. Right: residual map. Contour levels as in Fig. B.1. The blue colour scale is used for the [O I] $63 \mu \mathrm{m}$ map and the purple colour scale for the [O I] $146 \mu \mathrm{m}$ and [C II] $158 \mu \mathrm{m}$ maps. The Herschel PSF for each observation is shown in the top left corner.

Total flux
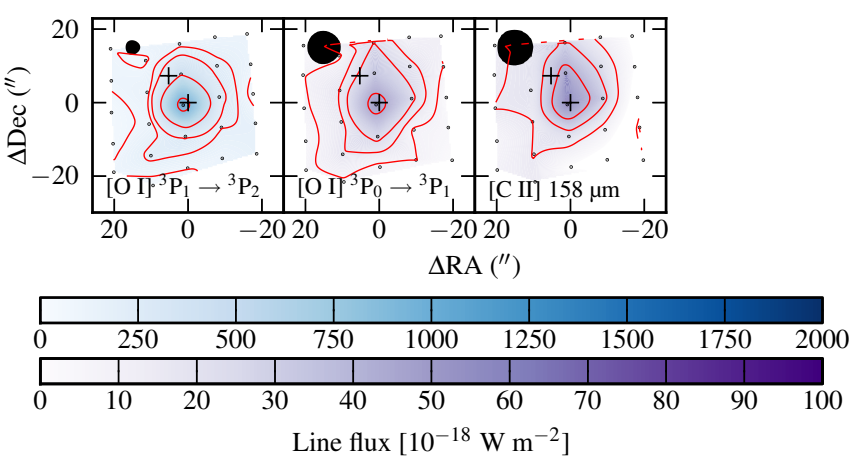

Residual flux
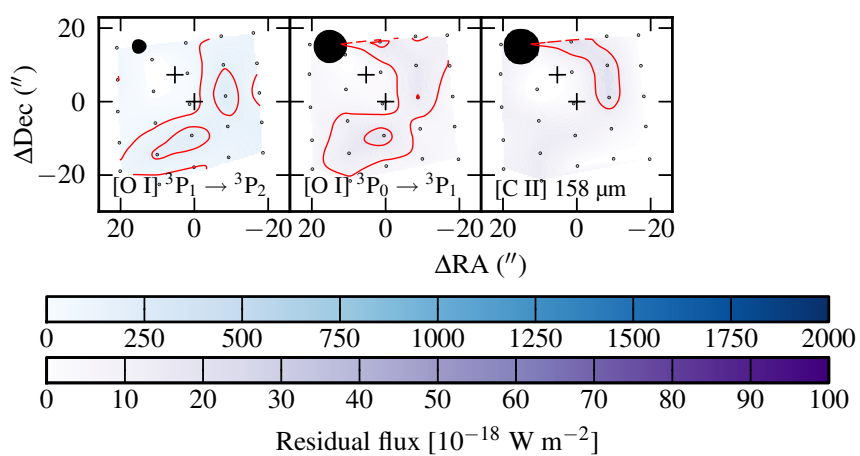

Fig. B.10. Atomic line maps of the IRS5 region. Left: total flux map. Right: residual map. Contour levels as in Fig. B.1. The blue colour scale is used for the [O I] $63 \mu \mathrm{m}$ map and the purple colour scale for the [O I] $146 \mu \mathrm{m}$ and [C II] $158 \mu \mathrm{m}$ maps. The Herschel PSF for each observation is shown in the top left corner. 


\section{Appendix C: The POMAC deconvolution algorithm}

POMAC (Poor Man's CLEAN) is a modification of the CLEAN algorithm (Högbom 1974), adapted to deconvolve data with a small number of data points, using previous knowledge about point-source positions as a restricting assumption. The user needs to define the positions of possible point sources (and point sources can be added or removed after running the script, iteratively refining the used set of point sources). The algorithm iteratively subtracts emission corresponding to the pre-defined point sources, finally leaving a residual map showing any emission not attributed to these point sources (extended emission or emission from previously unknown point sources).

More specifically, the script creates an $n \times n$ grid (we use $n=400$, giving a pixel size of $\sim 0.2$ for the IRS7 map) for each of the point sources, covering the whole field of view of the PACS footprint(s). For each of the grids, a unitary point source is convolved with a simulated telescope PSF. We use the simulated Herschel PSF, which is available for 60, 70, 80, 90, 100, 120, 140, 160, 180, and $200 \mu \mathrm{m}^{5}$ (see Fig. C.1). We linearly interpolate these for the intermediate wavelengths. The script iterates over each grid point to determine which PACS spaxel(s) the signal in this grid point will fall into (zero or one spaxels per PACS footprint). The result of this is one PACS instrument PSF (dirty beam) for each of the point sources in the field. We assume PACS spaxel sizes of 9.' $4 \times 9$ !' 4 , centred at the coordinates given by the telescope data, oriented parallel to the PACS grid. This is, however, a simplified model of the true spaxel footprint, but the simplification will not affect the results more than the calibration accuracy of the instrument $(20 \%)$.

The line flux measured in each of the spaxels is put in a residual flux matrix. Next, the script determines which spaxel is closest to each of the point sources. The script then analyses the residual map flux in each of these spaxels, and based on the distances of the point sources to their nearest spaxels the brightest point source is determined. A user-defined CLEAN gain (we use 0.01) is multiplied to the calculated flux and added to the CLEAN flux of this point source. The convolution of the PACS instrument PSF and a point source with the CLEANed flux is then subtracted from the residual map. This is iterated until one of several possible stop criteria is met:

1. The strongest point-source residual corresponds to negative flux.

2. The residual flux in any one spaxel near a point-source position drops below $-3 \sigma$.

3. The residual fluxes in any two spaxels drop below $-3 \sigma$.

4. The residual flux drops below $1 \sigma$ in a spaxel that is associated with a point source.

5. The average of the residual map drops below 0 .

6. The set maximum for CLEAN iterations is reached (this criterion was not reached in any of our deconvolutions).

After this is finished, the flux of each point source is found in the CLEAN flux vector, and the remaining residual map can be

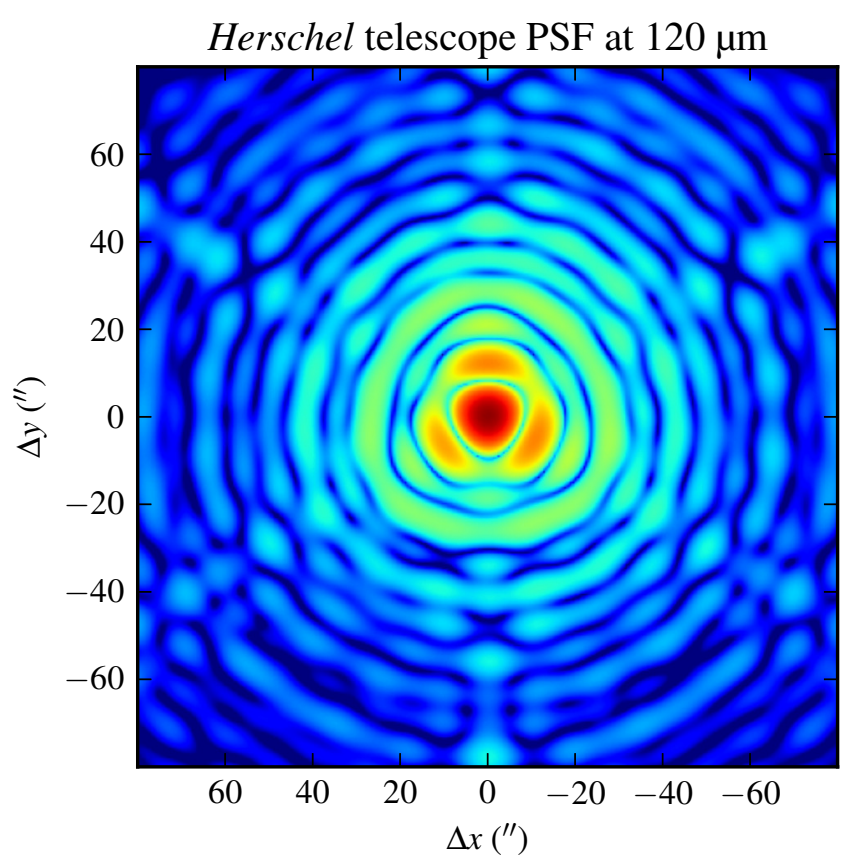

Fig. C.1. The Herschel simulated PSF at $120 \mu \mathrm{m}$ plotted with logarithmic scaling to better show the side lobes.

investigated to search for more point sources or extended emission (we note, however, that the residual map is still convolved with the instrument and telescope PSF).

The algorithm was tested on well-centred PACS data of the disc source HD 100546 (Sturm et al. 2010), which is assumed to behave like a point source in the continuum. The residuals after removing the central point source from the continuum data were found to be less than $5 \%$ of the extracted flux density across the whole spectrum.

It should be noted that in the case of extended emission across the whole field, the algorithm will extract too much flux to the point source, producing a hole in the residual emission. It is, however, impossible to predict the amount of the emission on the point source that should be attributed to the extended emission, and we have thus chosen the simplest approach. In the CrA data, the large-scale extended emission is weak in comparison with the point source emission, so this does not appear to affect the results by more than $20 \%$.

The algorithm works particularly well in the IRS7 field, where we have two overlapping PACS footprints (creating a grid that almost is Nyquist-sampled for most of the PACS bandwidth), but it is also useful for regions with only one PACS footprint, such as IRS5. The method is useful, not only for disentangling emission from several point sources, but also for establishing whether a source is a point source or shows extended emission.

\footnotetext{
5 Zemax modelled PSFs, which are found to agree remarkably well with verification observations:

http://herschel.esac.esa.int/twiki/pub/Public/

PacsCalibrationWeb/PACSPSF_PICC-ME-TN-029_v2.0.pdf
} 


\section{Appendix D: SED flux densities}

The flux densities from the literature used in the SED fits in Sect. 4.1 are listed in Table D.1.

Table D.1. Flux densities used in the SED fits in Sect. 4.1.

\begin{tabular}{|c|c|c|c|}
\hline $\begin{array}{l}\text { Name } \\
\text { Telescope }\end{array}$ & $\begin{array}{l}\text { Wavelength } \\
{[\mu \mathrm{m}]}\end{array}$ & $\begin{array}{l}\text { Flux density } \\
{[\mathrm{Jy}]}\end{array}$ & Reference \\
\hline \multicolumn{4}{|l|}{ IRS7A } \\
\hline \multirow[t]{4}{*}{ Spitzer } & 3.6 & 0.0509 & \multirow[t]{4}{*}{ Peterson et al. (2011) } \\
\hline & 4.5 & 0.239 & \\
\hline & 5.8 & 0.546 & \\
\hline & 8.0 & 1.05 & \\
\hline \multicolumn{4}{|l|}{ SMM 1C } \\
\hline \multirow[t]{2}{*}{$\mathrm{JCMT} \mathrm{SCUBA}^{a}$} & 450 & 45 & \multirow{2}{*}{ Nutter et al. (2005) } \\
\hline & 850 & 5.6 & \\
\hline \multicolumn{4}{|l|}{ IRS7B } \\
\hline \multirow[t]{4}{*}{ Spitzer } & 3.6 & 0.0367 & \multirow[t]{4}{*}{ Peterson et al. (2011) } \\
\hline & 4.5 & 0.257 & \\
\hline & 5.8 & 0.697 & \\
\hline & 8.0 & 0.957 & \\
\hline \multirow[t]{2}{*}{$\mathrm{JCMT} \mathrm{SCUBA}^{a}$} & 450 & 50 & \multirow[t]{2}{*}{ Nutter et al. (2005) } \\
\hline & 850 & 5.4 & \\
\hline \multicolumn{4}{|l|}{$\mathrm{R} \mathrm{CrA}$} \\
\hline \multirow[t]{5}{*}{ SAAO } & 0.36 & 0.0141 & \multirow[t]{5}{*}{ Koen et al. (2010) } \\
\hline & 0.45 & 0.0359 & \\
\hline & 0.555 & 0.0647 & \\
\hline & 0.67 & 0.116 & \\
\hline & 0.87 & 0.180 & \\
\hline \multirow[t]{3}{*}{ 2MASS } & 1.25 & 2.68 & \multirow[t]{3}{*}{ Skrutskie et al. (2006) } \\
\hline & 1.65 & 10.7 & \\
\hline & 2.2 & 47.9 & \\
\hline \multicolumn{4}{|l|}{ IRS5A } \\
\hline \multirow[t]{4}{*}{ Spitzer } & 3.6 & 0.389 & \multirow{4}{*}{ Peterson et al. (2011) } \\
\hline & 4.5 & 0.798 & \\
\hline & 5.8 & 1.27 & \\
\hline & 8.0 & 1.81 & \\
\hline \multicolumn{4}{|l|}{ IRS5N } \\
\hline \multirow[t]{4}{*}{ Spitzer } & 3.6 & 0.00856 & \multirow[t]{4}{*}{ Peterson et al. (2011) } \\
\hline & 4.5 & 0.0262 & \\
\hline & 5.8 & 0.0528 & \\
\hline & 8.0 & 0.0809 & \\
\hline \multirow[t]{2}{*}{ JCMT SCUBA $^{a}$} & 450 & $12^{b}$ & \multirow{2}{*}{ Nutter et al. (2005) } \\
\hline & 850 & $1.8^{b}$ & \\
\hline
\end{tabular}

Notes. ${ }^{(a)}$ The integrated flux density in apertures of $16^{\prime \prime} \times 16^{\prime \prime}$ (SMM 1C), $18^{\prime \prime} \times 17^{\prime \prime}$ (IRS7B), and $24^{\prime \prime} \times 24^{\prime \prime}$ (IRS5N) are given. ${ }^{(b)}$ This is the combined value for IRS5A and IRS5N, which are unresolved. For the SEDs, we assign half of the flux density to each source. 